FACULTAD DE CIENCIAS ASTRONÓMICAS Y GEOFÍSICAS DE LA PLATA Instituto de Astrofísica de La Plata

\title{
Poblaciones estelares como laboratorios de física fundamental
}

\author{
Lic. Brenda E. Melendez \\ Director: Dr. Leandro Gabriel Althaus \\ Codirector: Dr. Marcelo Miguel Miller Bertolami
}

AÑo 2017

Tesis presentada para optar por el grado de Doctor en Astronomía 



\title{
Poblaciones estelares como laboratorios de física fundamental
}

\author{
RESUMEN
}

En esta Tesis se estudia la posibilidad de utilizar las poblaciones estelares como laboratorios de física fundamental con el objetivo de extraer las propiedades físicas de la partícula hipotética denominada axión. Esta partícula permite dar solución al problema fuerte de Carga-Paridad, a la vez que proporciona un candidato a materia oscura. La constante de acoplamiento axión-fotón $\left(g_{a \gamma \gamma}\right)$ en el contexto del modelo teórico KSVZ es acotada incorporando el efecto Primakoff en el interior de estrellas de masa intermedia y requiriendo la desaparición completa de la fase de blue loop, la cual es necesaria para dar cuenta de la existencia de estrellas Cefeidas Clásicas. Considerando distintos escenarios para los parámetros asociados al modelado de los bordes convectivos durante la quema de He, se determina hasta qué punto la cota para $g_{a \gamma \gamma}$ se degrada, y se establece una cota robusta para dicho acoplamiento. La cota superior más restrictiva obtenida es $g_{10} \equiv g_{a \gamma \gamma} 10^{10} \mathrm{GeV}=0.6$. Sin embargo, teniendo en cuenta las distintas cotas que surgen al variar los parámetros asociados a los bordes convectivos, se concluye que la cota superior más conservativa es $g_{10}=2$.

El acoplamiento axión-electrón en el modelo teórico DFSZ, por su parte, es explorado a partir del análisis detallado de la función de luminosidad de enanas blancas (WDLF) del disco Galáctico. Específicamente, se computan nuevas WDLF teóricas basadas en un tratamiento autoconsistente de la emisión de axiones en los modelos evolutivos, lo que permite dar cuenta del impacto de dicha emisión en la estructura térmica de la enana blanca. Asimismo, nuevas cotas para la masa del axión $\left(m_{a}\right)$ son derivadas mediante la comparación cuantitativa entre las WDLFs teóricas, calculadas suponiendo distintos valores de $m_{a}$ y métodos de normalización, y diferentes derivaciones de la WDLF observada. Finalmente, con el conocimiento actual de la WDLF, se muestra en la presente Tesis que es posible descartar masas por encima de los $10 \mathrm{meV}$. 



\section{Índice general}

1. INTRODUCCIÓN 1

1.1. Motivación y Objetivos . . . . . . . . . . . . . . . . . . . 1

1.2. Argumento de Pérdida de Energía . . . . . . . . . . . 5

1.3. Enanas Blancas . . . . . . . . . . . . . . . . . . . . . . . . . . 7

1.4. Estrellas Cefeidas . . . . . . . . . . . . . . . . . . . . . . . . 19

2. Axiones 25

2.1. El problema fuerte de Carga-Paridad . . . . . . . . . . . . . . . . 25

2.2. El modelo de Peccei-Quinn _ . . . . . . . . . . . . . . . . . . 27

2.3. Modelos fenomenológicos de axiones _ . . . . . . . . . . . . . . . . . . . 29

2.4. Implementación de rutinas para la emisión de axiones en el código de evolución estelar LPCODE. . . . . . . . . . . . . . . . . . . . . . 36

2.5. Cotas existentes . . . . . . . . . . . . . . . . . . . . . 39

3. Cotas para la CONSTANTe De ACOPlamiento aXión-Fotón a PARTiR De estrellas Cefeidas $\quad 49$

3.1. Herramientas Numéricas: Física constitutiva y secuencias evolutivas . . . 51

3.2. Comparación con el código MESA _ . . . . . . . . . . . . . . 53

3.3. Impacto de la emisión de axiones durante la evolución . . . . . . . . . 54

3.4. Cotas derivadas a partir de la desaparición del Blue Loop . . . . . . . . . 58

4. Cotas para el axión derivadas de la WDLF Galáctica 63

4.1. Física de entrada y modelos iniciales de EBs . . . . . . . . . . . . . . . 64 
4.2. Función de luminosidad de EBs . . . . . . . . . . . . . 65

4.3. Impacto de la emisión de axiones en el enfriamiento de las EBs . . . . . 67

4.4. Impacto de la emisión de axiones en la WDLF . . . . . . . . . . . . . 69

5. Conclusiones y Trabajo a Futuro 77

Apéndice A. Código de Evolución Estelar LPCODE 83

A.1. Detalles Numéricos . . . . . . . . . . . . . . . . . . . . . . . 83

A.2. Tratamiento de la ecuación de cambio químico . . . . . . . . . . . . 85

$\begin{array}{ll}\text { REFERENCIAS } & 103\end{array}$ 
A la MEMoRia de Mi PAPÁ, YAYO. 



\section{Agradecimientos}

En primer lugar agradezco a Marcelo y Leandro por el apoyo académico otorgado durante estos años. A mis amigos de la oficina Nelly y Fran, por hacer las horas de trabajo más llevaderas y agradables. También quiero agradecer a mis amigas astronómas y físicas: Ale, Juanita, Flor, Marina, Juliana y las demás "Lulus" por los momentos compartidos y por hacer de esta profesión una actividad humana más cálida.

Especialmente agradezco a mis amigos del alma: Nadia, Gustavo, Lau y Maca, por prestarme su oido y su corazón siempre que lo necesité, a mi segunda familia: Mabel, Hugo, Martín, Ivi y Charly, por brindarme su gran amor en los momentos de mayor dificultad, y a mi mamá, que con su esfuerzo y humildad me permitió estudiar y conocer la astronomía. Por último, este camino habría sido imposible sin el apoyo incondicional, la confianza y el amor de Nico. 



\section{1 \\ Introducción}

\subsection{Motivación y OBjetivos}

Desde que Newton propuso que las leyes de movimiento que regían a los astros y a los objetos terrestres eran las mismas, los astros han sido utilizados múltiples veces para testear nuevas teorías físicas. El último siglo no ha sido una excepción a esta tradición: la confirmación de la Teoría General de la Relatividad por Eddington en 1919, del principio de exclusión de Pauli proveída por Fowler en 1926 al identificar a los electrones degenerados como la fuente de presión en el interior de enanas blancas o las masas límites de estrellas de neutrones y enanas blancas como confirmación de las predicciones de las teorías de la relatividad y la mecánica cuántica son ejemplos históricos de esta tradición. Ejemplos más recientes son las determinaciones de la necesidad de incorporar materia oscura y energía oscura en nuestra descripción del universo proveídas por el estudio de las curvas de rotación galácticas y las luminosidad de SNeIa. En lo que hace a la física de partículas el problema de los neutrinos solares, ofrece un ejemplo claro de como las estrellas pueden utilizarse para la detección de nueva física. Así por ejemplo, a fin de explicar las observaciones de los neutrinos solares ha sido necesario extender el modelo estándar de partículas fundamentales para incorporar una masa no nula para 
los neutrinos, los cuales, a su vez, deben poseer autoestados de masa diferentes de los autoestados de sabor. Asimismo, las actuales mejoras en el modelado y observaciones concernientes al Sol y otras estrellas permiten utilizarlas como laboratorio de física fundamental.

La presente Tesis se enmarca en esta tradición de utilizar a los cielos como laboratorio para testear la física fundamental. Específicamente, en esta Tesis se estudia el impacto producido por la existencia de una partícula liviana denominada "axión" sobre las propiedades observables de algunos grupos de estrellas, más concretamente de las estrellas variables de tipo Cefeidas Clásicas y de las Enanas Blancas. El axión surge como consecuencia de una de las soluciones más aceptadas para explicar la conservación de la simetría CP en las interacciones fuertes, ver Peccei \& Quinn (1977a) y Peccei \& Quinn (1977b), y es uno de los candidatos a materia oscura más justificados teóricamente. Aunque esta nueva partícula no ha sido aún descubierta, el rango permitido de parámetros necesarios para acotar su masa esta fuertemente restringido por consideraciones astrofísicas, cosmológicas y medidas de laboratorio.

La astrofísica brinda un laboratorio ideal para estudiar este tipo de partículas elementales ya que, en el caso de los axiones, el rango de energías térmicas (del orden de los $\mathrm{KeVs}$ ) presente en las estrellas permiten hacer los tests necesarios para estudiar sus propiedades. Las estrellas son además fuentes de neutrinos, entre otras partículas, producidos a partir de reacciones nucleares o procesos térmicos. Si una cantidad de energía considerable se perdiera ya sea a través de la emisión de nuevas partículas débilmente interactuantes o de la emisión de neutrinos con nuevas propiedades (momento dipolar magnético no nulo por ejemplo), entonces es de esperar que las propiedades evolutivas de las poblaciones estelares se vean fuertemente modificadas. Este es el llamado "argumento de pérdida de energía", ver Raffelt (1996), utilizado para acotar las propiedades de las partículas.

En esta Tesis estudiamos dos tipos de modelos de axiones invisibles discutidos con mayor frecuencia en la literatura: KSVZ, Kim (1979) y Shifman et al. (1980), y DFSZ, Zhitnitskii (1980) y Dine et al. (1981). En el primer caso, los axiones se acoplan exclusivamente a quarks pesados a través de un vértice de dos fotones. Este acoplamiento resulta proporcional a su masa, que es un parámetro libre del modelo. Por otra parte, 
en el segundo caso los axiones se acoplan a electrones o quarks livianos. El acoplamiento de los axiones DFSZ a electrones es también proporcional a su masa de forma análoga a los axiones KSVZ.

Como es sabido, el acoplamiento KSVZ de axiones con dos fotones permite las transiciones entre éstos y los fotones en un campo eléctrico o magnético. Además, según Carosi et al. (2013), estas partículas débilmente interactuantes poseen un acoplamiento muy débil con los campos anteriormente mencionados y su densidad lagrangiana * puede ser escrita de la siguiente manera

$$
\mathcal{L}_{a \gamma \gamma}=-g_{a \gamma \gamma} a \bar{E} \cdot \bar{B}
$$

donde $g_{a \gamma \gamma}$ es la constante de acoplamiento entre axiones y fotones, $a$ es el campo del axión y $\bar{E}$ y $\bar{B}$ son los campos eléctricos y magnéticos respectivamente. Por otra parte, la intensidad del acoplamiento con fotones está descripto por $g_{a \gamma \gamma}$ que se relaciona con la masa del axión " $m_{a}$ ", de la siguiente manera (ver Friedland et al. (2013))

$$
g_{a \gamma \gamma}=3.7 \times 10^{-10} \mathrm{GeV}\left(\mathrm{m}_{\mathrm{a}} / 1 \mathrm{eV}\right)
$$

Según Raffelt (1990) el lagrangiano que describe la interacción de un axión con un fermión de la especie " $j$ ", i.e. el electrón, puede escribirse como:

$$
\mathcal{L}_{a j}=-i g_{a j} \bar{\psi}_{j} \gamma_{5} \psi_{j} a
$$

En la ec. (1.3), $g_{a \gamma}$ representa la constante de acoplamiento de Yukawa adimensional, $\psi_{j}$ es el campo del fermión, $\bar{\psi}_{j}=\psi_{j}^{\dagger} \gamma^{0}$ y $\gamma^{5}=i \gamma^{0} \gamma^{1} \gamma^{2} \gamma^{3}$ es una de las matrices de Dirac.

Para los axiones de este tipo (DFSZ) el acoplamiento con electrones está definido por la intensidad del acoplamiento axión-electrón, $g_{a e}$, que se relaciona también con la

\footnotetext{
* La densidad lagrangiana se define como el lagrangiano $(L)$ por unidad de volumen, de manera que $\int \mathcal{L} d^{3} x=L$.
} 
masa del axión a través de

$$
g_{a e}=2.8 \times 10^{-14} \times m_{a[\mathrm{meV}]} \times \cos ^{2} \beta
$$

donde $\cos ^{2} \beta$ es un parámetro que depende el modelo y $g_{a e}$ está referida a la constante de estructura fina axiónica $\alpha_{26}$ mediante la siguiente relación

$$
\alpha_{26}=10^{26} \times g_{a e}^{2} / 4 \pi
$$

Los axiones podrían jugar un rol importante en la historia del universo. Estos se podrían haber generado luego del Big-Bang y también en el núcleo de estrellas como Cefeidas y enanas blancas. Asimismo, los axiones producidos en el universo temprano podrían contribuir a la materia oscura fría. Particularmente, en la presente Tesis se estudian ambos tipos de modelos axiónicos y se revisan y rederivan cotas a las constantes de acoplamiento $g_{a \gamma \gamma}$ y $g_{a e}$.

En el caso de la constante de acoplamiento axión-fotón $g_{a \gamma \gamma}$ se reanaliza el impacto de la emisión de axiones en el interior de estrellas Cefeidas Clásicas propuesto por Friedland et al. (2013). Estos autores presentaron un estudio de la emisión de axiones de tipo KSVZ por efecto Primakoff en el cual se concluye que valores $g_{a \gamma \gamma}>0.8 \times 10^{-10} \mathrm{GeV}^{-1}$ harían imposible la mismísima existencia de las variables de tipo Cefeidas. Obteniendo así una cota, supuestamente robusta, de $g_{a \gamma \gamma}<0.8 \times 10^{-10} \mathrm{GeV}^{-1}$ para el acoplamiento axión-fotón. Sin embargo, este estudio ignora el impacto de las incertezas existentes en la teoría de estructura estelar en la robustez de esta cota. Especialmente el argumento utilizado por estos autores depende de características de los modelos de evolución estelar que son muy dependientes de los detalles del modelado de los bordes convectivos durante la quema central de helio, y en consecuencia bastante inciertas. En esta Tesis, por lo tanto, se determina hasta que punto se degrada la cota sobre $g_{a \gamma \gamma}$ obtenida mediante el argumento de Friedland et al. (2013) como consecuencia de las incertezas existentes en el tratamiento de los bordes convectivos en los modelos de estructura y evolución estelar. 
Las cotas a la constante de acoplamiento axión-electrón $g_{a e}$ de los modelos de tipo DFSZ se estudian mediante su impacto en el enfriamiento de enanas blancas, más específicamente sobre la función luminosidad de enanas blancas (WDLF) del disco Galáctico. Esta función representa el número de enanas blancas por unidad de volumen y luminosidad. Isern et al. (2008) determinó que la función luminosidad del disco Galáctico determinada por Harris et al. (2006) mostraba indicios de la existencia de un enfriamiento anómalo el cual podría corresponderse con la existencia de axiones DFSZ con masas de $m_{a} \sim 5 \mathrm{meV}$. Sin embargo, el análisis de estos autores fue realizado utilizado un enfoque perturbativo en el cual se despreciaba el impacto de la emisión de axiones sobre la estructura térmica de los modelos evolutivos de enanas blancas. Más específicamente, el impacto de la emisión de axiones sobre la temperatura interna y, como consecuencia, sobre la emisión de neutrinos fue ignorada. Este efecto tiene como consecuencia disminuir el impacto de la existencia de los axiones sobre el enfriamiento de enanas blancas, haciendo menos relevante su impacto sobre la función de luminosidad. Como consecuencia sería esperable una degradación de las cotas obtenidas al realizar el análisis mediante la utilización de modelos de enanas blancas que incluyesen la emisión de axiones de manera completamente autoconsistente. Este es uno de los objetivos perseguidos, y alcanzados, en la presente Tesis. Por otra parte, la mejora constante de los sondeos celestes como el Sloan Digital Sky Survey (SDSS) y el Super-Cosmos Sky Survey han permitido continuar mejorando las determinaciones observacionales de la función de luminosidad de enanas blancas, como también detectar diferencias sistemáticas entre aquellas funciones de luminosidad derivadas de manera independiente. En este contexto otro de los objetivos de esta Tesis es analizar hasta que punto las cotas a la constante de acoplamiento axión-electrón $g_{a e}$ son dependientes de los detalles de la normalización y determinación de la función de luminosidad de enanas blancas utilizada.

\subsection{Argumento de PÉrdida de Energía}

En 1940 los científicos Gamow y Schoenberg marcaron el inicio de la astrofísica de partículas al suponer que los neutrinos juegan un papel relevante en la evolución 
estelar, específicamente en el colapso de las estrellas. Más tarde, se comprobó que estas partículas solamente interactúan a partir de procesos $\beta$, e.g. los conocidos procesos URCA. Estas reacciones son importantes a temperaturas o densidades muy altas debido a sus umbrales energéticos. Subsecuentemente, se conocieron las emisiones dominantes de neutrinos desde un plasma estelar: bremsstrahlung $e^{-}+(A, Z) \rightarrow(A, Z)+e^{-}+\bar{\nu} \nu$, foto-neutrino $\gamma e^{-} \rightarrow e^{-} \bar{\nu} \nu$ y decaimiento de un fotón $\gamma \rightarrow \bar{\nu} \nu$.

El modelo estándar de partículas describe y explica la interacción entre partículas fundamentales de la materia bajo la presencia de las siguientes fuerzas: la fuerza electromagnética, la nuclear fuerte y la débil. El modelo estándar es una teoría de gauge que representa interacciones fundamentales a través del cambio en la función lagrangiana de los campos cuánticos. Además, éste contiene términos cinemáticos, acoplamientos y términos de interacción relacionados con simetrías de gauge de las fuerzas, términos de masa y términos que contienen el sector de Higgs (aquella parte del lagrangiano que involucra al campo de Higgs). Asimismo, para ciertas extensiones del modelo estándar la ruptura espontánea de simetría de este lagrangiano conlleva a la aparición de un bosón de masa muy baja, el bosón de Nambu-Goldstone de esta simetría. De esta manera, como se mencionó anteriormente, el axión es el bosón de la simetría de Peccei \& Quinn (1977b) que explica la conservación de "Carga-Paridad" (CP) en las interacciones fuertes.

Por otra parte, si consideramos la emisión de nuevas partículas al igual que la emisión de neutrinos, podremos observar el fuerte impacto sobre la evolución y propiedades estelares. Sato \& Sato (1975) utilizaron el argumento de "Pérdida de Energía" para obtener cotas en la constante de acoplamiento del famoso bosón de "Higgs" del modelo estándar. A partir de ello, extendieron el análisis para muchas partículas en distintos escenarios astrofísicos. Asimismo, se mostró que existe una modificación en los tiempos evolutivos debido a la emisión de partículas débilmente interactuantes. Por lo tanto, al considerar pérdidas de energías por axiones las estrellas, tales como las estudiadas en esta Tesis, consumen su combustible más rápido. Sin embargo, para el caso de gigantes rojas de baja masa la emisión retrasa la quema de He y de esta manera extiende este estadio, ver Raffelt (1996). De esta manera, es fundamental poseer un modelado teórico de la estructura estelar para determinar qué fases evolutivas son más sensibles al mecanismo 
de pérdida de energía.

La evolución estelar es una prueba importante para la física de partículas. En el plasma denso y caliente que existe en el interior de las estrellas ciertas partículas, como el axión, que interactúan muy débilmente como para ser observadas en laboratorios, podrían ser producidas de manera abundante. Debido a su débil interacción con la materia y la radiación, los axiones podrían escapar libremente del interior estelar llevándose consigo cierta cantidad de energía y por lo tanto podrían cambiar las propiedades de la evolución estelar, ver Raffelt \& Dearborn (1988). Más concretamente, es posible afirmar que el impacto de nuevas partículas sobre la evolución estelar se maximiza cuando el camino libre de la partícula es comparable a la estructura geométrica del sistema, ver Raffelt (1996). De esta manera, los estudios astrofísicos y observacionales son fundamentales como herramientas para obtener información sobre este tipo de partículas y sus interacciones. En este sentido, las poblaciones estelares son complementarias a los distintos laboratorios terrestres que contemplan la nueva física de neutrinos, axiones, fuerzas de largo alcance entre otras cosas, ver Raffelt (1999).

\subsection{EnANas Blancas}

Las Enanas Blancas (EBs) son la etapa final de la evolución de estrellas de masa baja e intermedia, es decir, masas estelares menores que $10 M_{\odot}$, las cuales constituyen más del $97 \%$ de todas las estrellas. Estos remanentes estelares no poseen fuentes apreciables de energía nuclear, de manera que, a medida que transcurre el tiempo, las EBs se enfrían lentamente radiando su reserva de energía térmica, por lo que se vuelven cada vez menos luminosas.

La principal motivación para el estudio de las EBs radica en que el mismo tiene potenciales aplicaciones en diversos campos de la astrofísica. En primer lugar, la gran cantidad de datos observacionales de alta calidad respecto de las EBs, obtenidos tanto de observatorios espaciales como de telescopios terrestres, ha permitido la utilización de la secuencia de enfriamiento de estas estrellas como indicador independiente de edad y distancia para una vasta variedad de poblaciones estelares. Un simple cálculo muestra que el tiempo que las EBs toman en apagarse y desaparecer por encima de las capaci- 
dades de los telescopios actuales es muy largo, 10Gyr. Por tanto, el conocimiento preciso de la tasa a la cual las EBs se enfrían provee de un reloj cósmico independiente para constreñir la edad y la historia pasada de las distintas componentes Galácticas, incluyendo el disco y el sistema de cúmulos abiertos y globulares. Más aún, debido a que los progenitores de las EBs pierden sus capas externas (ricas en carbono, oxígeno e hidrógeno) en la parte superior de la rama asintótica de gigantes, las mismas contribuyen significativamente a la evolución química de la galaxia. Por otra parte, la posibilidad de explorar la distribución de masa de las EBs permitiría adquirir un mayor conocimiento de los procesos de pérdida de masa durante la evolución estelar y del balance de masa de la galaxia.

Las EBs con masas por debajo de $0.4 M_{\odot}$ son miembros de sistemas de binarios cercanos, en los cuales puede darse una transferencia de masa desde la estrella compañera hacia la EB, dando lugar a eventos muy energéticos (esto sucede, por ejemplo, en estrellas variables cataclísmicas). Asimismo, las EBs en sistemas binarios son candidatas a progenitores de supernovas de tipo Ia, de manera que el estudio de las propiedades estructurales y evolutivas de estas estrellas permite mejorar el conocimiento de eventos de supernova, lo que a su vez tiene importantes implicancias en el campo de la cosmología.

La naturaleza compacta de las EBs se traduce en una gran densidad media y gravedad superficial, y una baja luminosidad. A tan altas densidades, los electrones se vuelven degenerados y entonces la mecánica cuántica, más precisamente el principio de exclusión de Pauli, determina la ecuación de estado y la estructura de estas estrellas y establece una masa límite para la existencia de las mismas, ver Chandrasekhar (1939). Debido a estas propiedades, las EBs pueden considerarse como laboratorios cósmicos ya que permiten estudiar numerosos procesos físicos bajo condiciones extremas que no pueden ser reproducidas en laboratorios terrestres. Algunos ejemplos de estudios físicos que pueden desarrollarse desde este punto de vista son: la ecuación estado, cristalización y el proceso de separación química por decantamiento gravitacional a muy alta densidad, la emisión de neutrinos, la variación de constantes fundamentales e, íntimamente relacionado con lo hecho en esta Tesis, la existencia de axiones.

Finalmente, es preciso mencionar que, en el curso de su evolución, las EBs cruzan 
varias fases de inestabilidad pulsacional, por lo que también constituyen una herramienta potente para testear las regiones internas de las mismas, inaccesibles por observación directa. Más aún, las EBs pulsantes poseen aplicaciones que trascienden la astrofísica estelar. En efecto, dichas estrellas pueden ser usadas para poner límites a las propiedades de ciertas partículas elementales, tales como los neutrinos y el axión.

Teniendo en cuenta las distintas motivaciones para el estudio de las EBs mencionadas y, fundamentalmente, el hecho de que las mismas pueden ser utilizadas como laboratorios cósmicos para acotar las propiedades del axión, es conveniente repasar en primer lugar y someramente algunas características fundamentales de estas estrellas para luego sí detenerse con más detalle en el ingrediente principal utilizado en esta tesis para el estudio de la existencia del axión en el contexto de las EBs, a saber, la función de luminosidad de enanas blancas (WDLF).

\subsubsection{Breve Reseña Histórica de las Enanas Blancas}

En 1914, H. Russell descubrió que la estrella 40 Eridani B localizada justo por debajo de la secuencia principal en el diagrama H-R, estaba caracterizada por un radio pequeño comparado al radio terrestre (de allí la denominación de "enana"). El sorpresivo descubrimiento de Sirio B como una estrella EB llamó también la atención de la comunidad científica. Asimismo, Adams (1925) determinó el tipo espectral y la luminosidad de Sirio B y a partir de ello se pudo obtener la primera estimación del radio de esta estrella. Por otra parte, gracias a su masa (aprox. $1 \mathrm{M}_{\odot}$ ) fue posible obtener por primera vez su densidad media, aunque los valores actuales $\left(10^{6} \mathrm{~g} / \mathrm{cm}^{3}\right)$ son un orden de magnitud mayores debido a las discrepancias en la temperatura efectiva. Para esa época se pudo determinar el fuerte campo gravitacional de Sirio B, lo cual permitió confirmar la teoría de relatividad general a través de su corrimiento al rojo (el cual fue observado en laboratorio por Pound \& Rebka (1959)).

Alrededor de 1920, solamente tres EBs estaban catalogadas aunque ya se sospechaba que estos objetos podrían ser muy comunes en nuestra Galaxia. Estas estrellas estaban localizadas muy cerca del Sol, más precisamente alrededor de 5 pc de distancia del mismo. Para 1939 ya eran 18 las EBs conocidas y en 1950 el número se había incrementado a 111. Debido a que las EBs son intrínsecamente débiles, éstas eran observadas 
relativamente cerca del Sol y mostraban movimientos propios grandes con respecto al mismo. Por otra parte, una característica a destacar que presentaban estas estrellas es que eran demasiado azules para su luminosidad al compararlas con estrellas de secuencia principal. Esta propiedad fue estudiada exhaustivamente en el trabajo de Humason \& Zwicky (1947) y más tarde en el relevamiento de "Palomar-Green", ver Green et al. (1986).

Respecto del devenir histórico de la teoría de la ecuación de estado para las EBs, es preciso recordar primero que la descripción termodinámica de estas estrellas requiere de la mecánica cuántica y, por supuesto, de la mecánica estadística. En efecto, si se toman estimaciones actuales para la masa y radio de Sirio B, su densidad media resulta $\sim 3 \times 10^{6} \mathrm{gcm}^{-3}$. Así, asumiendo una composición interior de carbono puro se estima la distancia típica entre núcleos como $r_{c}=1.2 \times 10^{-10} \mathrm{~cm}$, la cual es mucho menor que el radio de Bohr para un ion de carbono con un único electrón adicional. Por tanto, no puede haber órbitas ligadas, y la materia en las EBs debe estar completamente ionizada por la presión. De manera análoga, la distancia típica entre electrones es $r_{e} \simeq$ $6.4 \times 10^{-11} \mathrm{~cm}$, la cual es menor que la longitud de onda térmica de de Broglie de los electrones para todas las temperaturas excepto las extremadamente altas $\left(>10^{9} \mathrm{~K}\right)$. La descripción de la materia bajo estas condiciones debe hacer uso de la mecánica cuántica.

Ahora bien, en 1926, Fermi y Dirac encontraron que los estados cuánticos de los electrones siguen una distribución estadística particular, hoy conocida como de FermiDirac,(ver Fermi (1926); Dirac (1926)). Esto fue aplicado inmediatamente por Fowler (1926), quien notó que la presión que soportan las EBs contra la gravedad es proporcionada por los electrones casi completamente degenerados. Más tarde, Anderson (1929) y Stoner (1930) dieron cuenta de que, bajo las condiciones de extrema densidad que se da en las EBs más masivas, las velocidades deben volverse relativistas y entonces calcularon correcciones relativistas a la ecuación de estado derivada por Fowler. A partir de esto, Chandrasekhar (1931) descubrió la existencia de un límite, conocido en la actualidad como "límite de Chandrasekhar", en el que las fuerzas gravitacionales sobrepasan la presión de sustento y entonces ninguna EB estable no rotante puede existir. En el caso de EB rotantes, para más detalle, ver (Hachisu et al., 2012). Esta conclusión también fue obtenida independientemente por Landau en 1932. Durante los 
ocho años posteriores, Chandrasekhar elaboró una teoría completa de la ecuación de estado a toda densidad, incluyendo tanto efectos de temperaturas finitas como de relatividad especial, y la correspondiente estructura de modelos de temperatura cero y su relación masa-radio, ver Chandrasekhar (1939).

\subsubsection{FunCiÓN DE MASA DE LAS EB}

Como se mencionó anteriormente, es importante destacar que las enanas blancas constituyen el producto final de todas las estrellas con masas de $\lesssim 10 M_{\odot}$, ver Siess (2007). En cuanto a su masa, esta está contenida principalmente en el núcleo de la estrella. Ahora bien, si tomamos en cuenta la historia termonuclear y el decantamiento (settling) gravitacional, es de esperar que la EB tenga una masa de $\sim 0.6 M_{\odot}$ y una estructura estratificada en composición. Más concretamente, dicha estructura consiste en un núcleo de $\mathrm{C}-\mathrm{O}$ rodeado de una envoltura fina de He de como máximo $\sim 0.01 M_{\odot}$, la cual se encuentra a su vez rodeada por una capa más fina aún de $\mathrm{H}$ de $\sim 10^{-4} M_{\odot}$.

Una propiedad importante de la población de EBs es su distribución de masa. Según Liebert et al. (2005), esta función contiene información respecto de nuestra Galaxia y está correlacionada con la masa de las estrellas en secuencia principal. La temperatura efectiva y la gravedad superficial de las EBs se determinan usualmente de los modelos de atmósferas. En promedio, las EBs se caracterizan por poseer gravedades superficiales con valores cercanos a $\log g \simeq 8 \mathrm{y}$, a partir de relaciones teóricas de masa-radio, se obtiene una masa promedio de $M \simeq 0.6 M_{\odot}$.

Actualmente, relevamientos tales como el "Sloan Digital Sky Survey" (SDSS) han permitido expandir el número de EBs espectroscópicamente identificadas y han dado a lugar al descubrimiento de EBs ultrafrías, EBs pulsantes ricas en H y EBs deficientes en H. Kepler et al. (2017) estimaron la masa de todas las EBs DA relevadas por Kleinman et al. (2013), Kepler et al. (2015) y Kepler et al. (2016) entre 4.5 millones de espectros obtenidos del SDSS Data Release 12. Debido a la gran cantidad de EBs detectadas se ha mejorado tanto la función luminosidad como la función distribución de masa de estas estrellas. La figura 1.1 muestra distribuciones de masa típicas obtenidas a partir de los relevamientos mencionados para EBs DA y DB. Como puede apreciarse, las masas de las EBs se encuentran distribuidas alrededor del valor medio comentado más arriba 
$\left(M \simeq 0.6 M_{\odot}\right)$ con una cola que se extiende hacia altas masas estelares. Se ha sugerido en Guerrero et al. (2004) que tales EBs podrían ser el resultado del merger de dos EBs de masa promedio en binarias cercanas. Además, la distribución de masa de las EBs abarca una población de remanentes de baja masa. Como los progenitores de baja masa necesitarían mucho tiempo para alcanzar el estadio de EB, éstas se producen en sistemas binarios donde existe transferencia de masa.

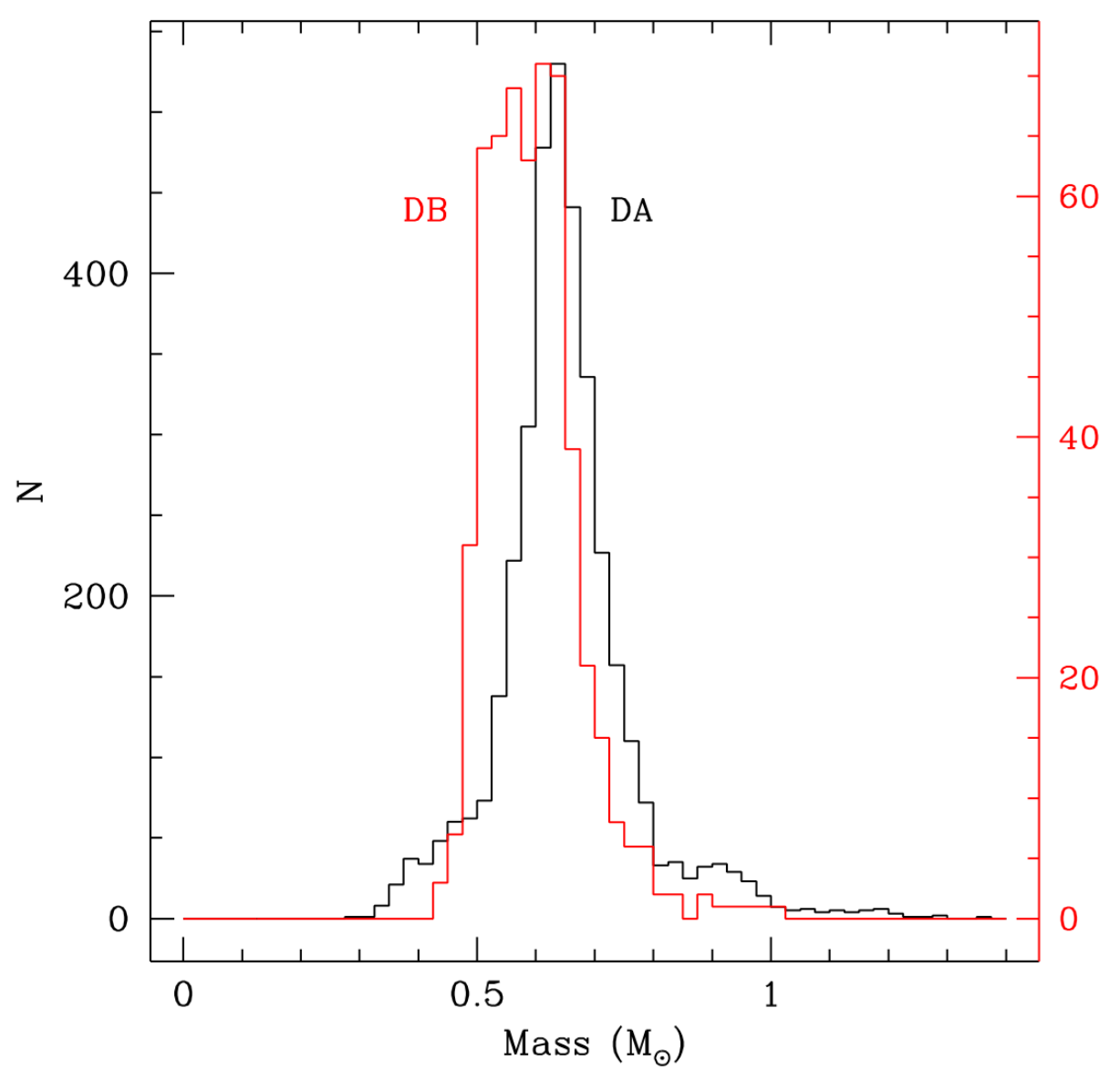

Figura 1.1: Función distribución de masa para $3636 \mathrm{~EB}$ DAs con $T_{\text {eff }}>13000 \mathrm{~K}$ representadas por líneas negras y $549 \mathrm{~EB}$ DBs con $T_{\text {eff }}>16000 \mathrm{~K}$ representadas por líneas rojas, ver 201.

\subsubsection{Clasificación Espectroscópica}

Desde el punto de vista fenomenológico, las EBs se clasifican en dos familias distintas de acuerdo al principal constituyente de sus respectivas atmósferas. Las denominadas 
DA comprenden aproximadamente el $85 \%$ del total de EBs y son aquellas cuya composición consiste casi enteramente en H, quizá con algunas trazas de otros elementos. EBs de este tipo espectral presentan líneas de Balmer detectables en el rango completo de temperaturas efectivas cubierto en el diagrama HR. El restante $15 \%$ de la población de EBs, con deficiencia de H y atmósferas ricas en He, es conocido como EBs "no DA". Este tipo de EBs parece provenir de mergers y de estrellas post-AGB que sufren un flash de He muy tardío el cual las empuja de vuelta a la AGB en lo que se conoce como escenario de "born-again". Esto produce un violento episodio de mezcla durante el cual el $\mathrm{H}$ residual es quemado, dando lugar a objetos muy calientes denominados estrellas PG1159, cuyas atmósferas se componen de una mezcla dominada por He, C y O en aproximadamente iguales proporciones. A medida que dichas estrellas se enfrían tiene lugar la sedimentación lo que produce EBs con atmósferas de He. Dependiendo la temperatura efectiva y la posible presencia de trazas de elementos contaminantes, las EBs no DA se dividen usualmente en varias subclases:

- Las de tipo espectral DO con temperaturas efectivas en el rango $45000 \mathrm{~K} \leq T_{\text {eff }} \leq$ $100000 \mathrm{~K}$, que exhiben líneas relativamente fuertes de HeII.

- Las de tipo espectral DB con temperaturas efectivas en el rango $11000 \mathrm{~K} \leq T_{\text {eff }} \leq$ 30000 K y líneas fuertes de HeI.

- Las de tipo DC, DQ y DZ con temperaturas por debajo de los $11000 \mathrm{~K}$, las cuales muestran trazas de carbono y metales en sus espectros.

A medida que las EBs de tipo DO evolucionan, el HeII se recombina y forma HeI, por lo que se transforman en EBs DB. Esta transición es interrumpida por el denominado gap DB, es decir, una zona entre 30000 K y 45000 K donde se da una escasez de EBs con atmósferas ricas en He, ver Eisenstein et al. (2006). A esta clasificación deben añadirse aquellas EBs con atmósferas híbridas o abundancias peculiares, el descubrimiento de dos EBs con atmósferas ricas en oxígeno, ver Gänsicke et al. (2010), y un nuevo tipo espectral llamado DQ caliente con una atmósfera dominada por carbono y $T_{\text {eff }} \sim 20000 \mathrm{~K}$, ver Dufour et al. (2007).

Por último, es importante destacar que existe una amplia evidencia observacional de que la composición superficial de una dada EB puede cambiar a medida que evoluciona, 
es decir, que existe la posibilidad de evolución espectral. Esta evolución es el resultado de la interrelación de procesos que compiten tales como la convección, episodios de pérdida de masa, acreción, levitación radiativa, y el decantamiento gravitacional. Tanto el cambio con $T_{\text {eff }}$ del cociente entre estrellas DA y no DA como la existencia del gap DB mencionado antes constituyen ejemplos de evidencia empírica que puede interpretarse en términos de transiciones desde el tipo DA hacia el tipo no DA y viceversa.

\subsubsection{EnFriamiento de las EnAnas Blancas}

La evolución de las EBs es esencialmente un proceso de enfriamiento. En general, la contribución a la luminosidad debida a fotones, neutrinos y partículas exóticas puede ser descripta de la siguiente manera

$$
L+L_{\nu}+L_{X}=-\int_{O}^{M_{W D}} C_{v} \frac{d T}{d t} d m-\int_{O}^{M_{W D}} T\left(\frac{\partial P}{\partial T}\right)_{V} \frac{d V}{d t} d m+\left(l_{s}+e_{s}\right) \dot{M}_{s}+\dot{\epsilon}_{X}
$$

donde el lado derecho de la ecuación representa el calor específico de la estrella, el trabajo compresional, la contribución de calor latente $\left(l_{s}\right)$, la energía liberada durante la cristalización $\left(e_{s}\right)$ multiplicada por su tasa $\dot{M}_{s}$ y finalmente el último término representa cualquier fuente adicional de energía exótica, ver el trabajo de Isern et al. (2010).

El enfriamiento de las EBs puede ser dividido en cuatro etapas. La primera es debido a los neutrinos, ésta se da para $\log \left(L / L_{\odot}\right)>-1.5$. En épocas tempranas, luego de atravesar la fase de nebulosa planetaria, el interior de las EBs es capaz de producir neutrinos en gran cantidad a partir de procesos que involucran interacciones débiles. La mayoría de los neutrinos escapan contribuyendo como sumidero de energía. La evolución de una EB caliente está dominada entonces por el enfriamiento de neutrinos. La evolución y estructura posterior depende exclusivamente de las propiedades de los electrones degenerados y los iones térmicos. La segunda etapa corresponde al enfriamiento como fluido para luminosidades en el rango de $-1.5>\log \left(L / L_{\odot}\right)>-3$. En esta etapa las pérdidas de energía son térmicas. El calor es conducido y transportado por conducción a través del interior degenerado donde luego es radiado por la envoltura de la estrella. Además, la difusión de energía, a través de la envoltura, depende fuertemente de la opacidad. La 
tercera etapa corresponde a los procesos de cristalización donde $\log \left(L / L_{\odot}\right)<-3$. Es allí donde se experimentan transiciones de fase de primer orden donde el núcleo profundo cristaliza a esas luminosidades. La cristalización introduce dos fuentes de energía nuevas: el calor latente y la energía gravitacional liberada por la separación de fase entre el C y O durante la cristalización. Además aparece una fuente de energía adicional, la difusión gravitacional del ${ }^{22} \mathrm{Ne}$ sintetizado del contenido inicial de carbono, nitrógeno y oxígeno durante la quema central de helio, ver Isern et al. (2013). Finalmente, la cuarta etapa corresponde al enfriamiento de Debye donde la estrella presenta un estado sólido. La red cristalina causa vibraciones coherentes que promueven la pérdida de energía. El enfriamiento de Debye es responsable de la evolución acelerada de las EBs más masivas a bajas luminosidades. En la figura 1.2 se muestran los distintos enfriamientos durante el estadio de enana blanca

\subsubsection{Función Luminosidad de Enanas Blancas}

Una herramienta fundamental para estudiar las propiedades de la población de las EBs es su función de luminosidad (WDLF), ver Althaus et al. (2010b). Esta función está definida como el número de EBs por parsec cúbico y por unidad de magnitud bolométrica (o luminosidad). La WDLF no sólo brinda información valiosa acerca de la edad, estructura, y evolución de nuestra Galaxia sino que también proporciona tests independientes para la teoría de plasmas densos, ver Isern et al. (1997) y Isern et al. (1998). Más aún, la WDLF acota la tasa de mortalidad de estrellas de masa baja o intermedia en el vecindario local. Por otra parte, como se mencionó anteriormente, debido a la simpleza de su estructura y evolución, las EBs resultan extremadamente útiles como laboratorios de astropartículas, ver Córsico et al. (2001). Sin embargo, a fin de explotar la utilidad de la WDLF en los problemas astrofísicos mencionados, es necesario contar con datos observacionales precisos, y modelos evolutivos realistas basados en una descripción física actualizada y completa.

En la actualidad, la fuente más importante de gran cantidad de nuevas EBs tanto en el disco como en el halo es el Sloan Digital Sky Survey (SDSS), ver York et al. (2000), el cual cuenta con alrededor de 10000 EBs identificadas (véase Harris et al. (2006), 


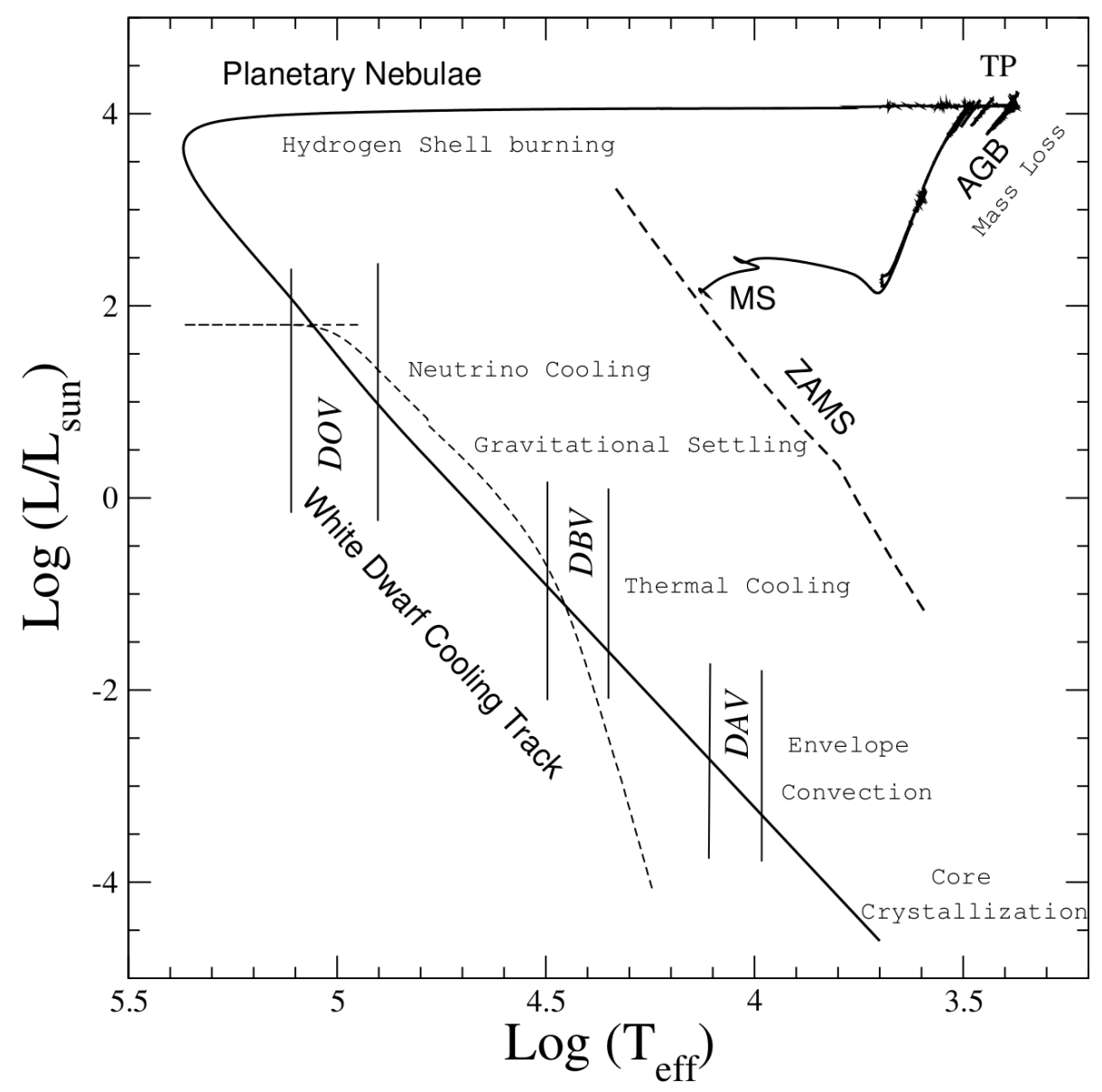

Figura 1.2: Diagrama $\mathrm{H}-\mathrm{R}$ para la evolución completa de una estrella de $3.5 \mathrm{M}_{\odot}$ desde la ZAMS hasta el estadio de EB. Se indican también los distintos tipos de enfriamiento y las bandas de inestabilidad para las EB pulsantes DOV, DBV y DAV. Figura tomada de Althaus et al. (2010b).

Eisenstein et al. (2006) y De Gennaro et al. (2008)). En el contexto de este último relevamiento, Harris et al. (2006) construyeron la WDLF a partir de una muestra de 6000 EBs DA y no DA con fotometría precisa y movimientos propios obtenida del SDDS Data Release 3. La figura 1.3 muestra que la correspondiente WDLF tiene una forma suave hasta $M_{b o l}=15.3$ donde presenta una caída pronunciada ("cut-off"). Mientras que el comportamiento monótono de la WDLF refleja esencialmente el enfriamiento de la EB, el abrupto cut-off es consecuencia de la edad finita de la Galaxia, ver Weidemann (1968). Con la finalidad de obtener mejoras en la WDLF, De Gennaro et al. (2008) construyeron esta misma función con una muestra de 3528 EB DA espectroscópicamente identificadas con el SDSS Data Release 4.

Teniendo en cuenta las distintas WDLFs disponibles en la literatura, es posible con- 


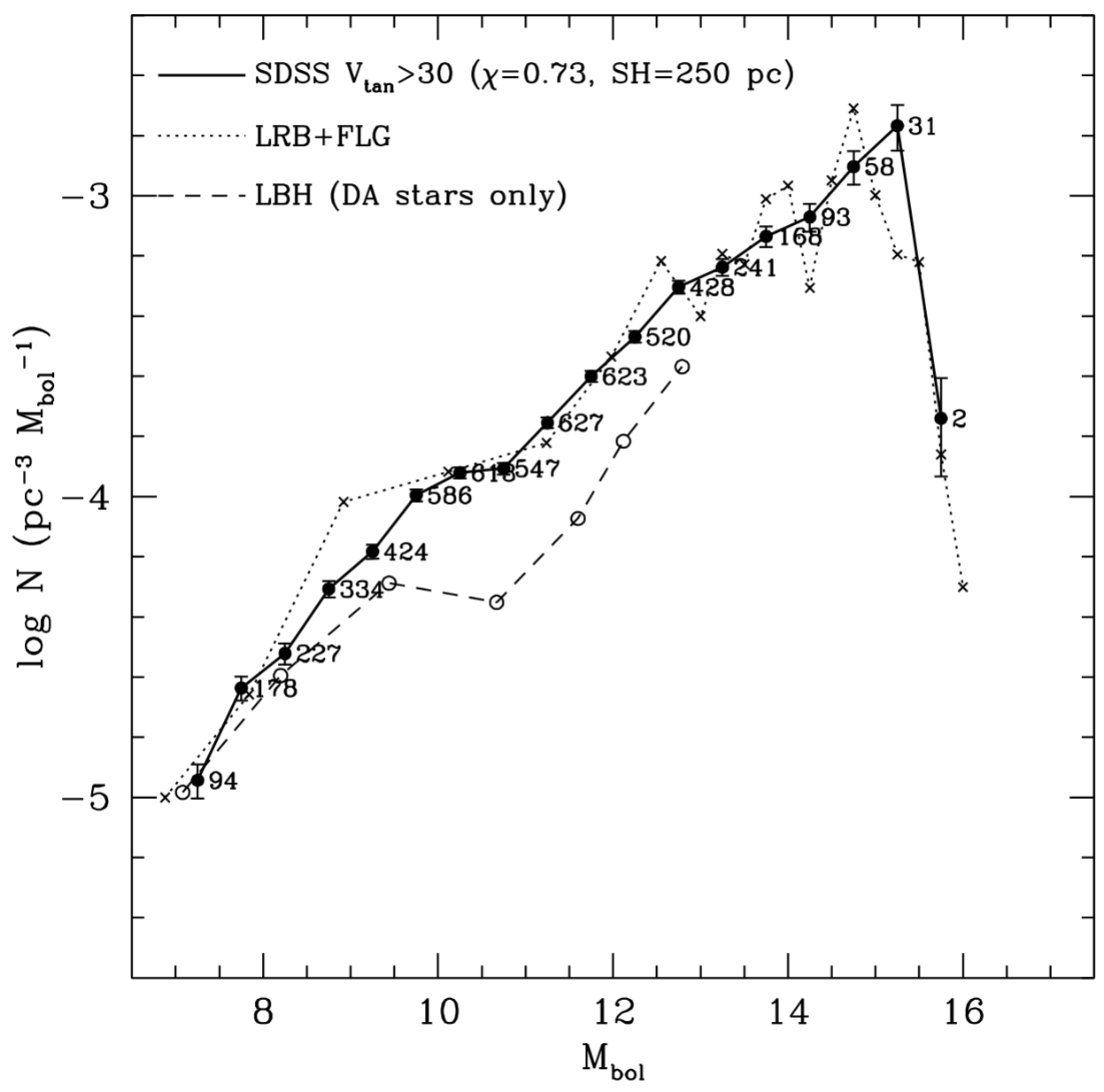

Figura 1.3: Comparación de la WDLF del disco derivada del Sloan Digital Sky Survey (SDSS) en Harris et al. (2006) con aquellas obtenidas en Leggett et al. (1998) y Liebert et al. (2005). Figura tomada de Althaus et al. (2010b)

cluir que, debido al tamaño de la muestra de EBs utilizada y al tratamiento cuidadoso de sus incertezas sistemáticas, la WDLF de Harris et al. (2006) constituye la mejor función de referencia contra la cual comparar nuevos estudios, ver García-Berro \& Oswalt (2016).

Ahora bien, a fin de determinar las propiedades evolutivas de las EBs es necesario conocer la forma de la WDLF teórica. Dicha forma teórica es construida a partir de la siguiente expresión 


$$
n\left(M_{b o l}\right)=\int_{M_{i}}^{M_{u}} \phi(M) \psi\left(T_{G}-t_{e n f}-t_{p}\right) \tau_{e n f} d M
$$

donde $M_{b o l}$ representa la magnitud bolométrica, $M$ es la masa de la estrella progenitora, $t_{\text {enf }}$ es el tiempo de enfriamiento necesario para alcanzar una determinada luminosidad $L, \tau_{e n f}=d t_{\text {enf }} / d M_{b o l}$ es el tiempo característico de enfriamiento, $t_{p}$ es el tiempo en secuencia principal del progenitor y, por último, $T_{G}$ es la edad de la población bajo estudio. Los límites de integración en la ec. (1.7) juegan un rol fundamental. El límite máximo $M_{u}$ es la masa máxima del progenitor capaz de formar una EB, mientras que el límite inferior $M_{i}$ es la mínima masa del progenitor en secuencia principal capaz de producir una EB con luminosidad L. A su vez, este límite inferior satisface la siguiente relación

$$
T_{G}-t_{e n f}-t_{p}=0
$$

Las cantidades restantes que aparecen en la ec. (1.7) son la función de masa inicial $\phi, y$ la tasa de formación estelar $\psi$, la cual no es conocida a priori y depende de la población estelar bajo estudio.

A fin de comparar las observaciones adecuadamente, es conveniente aplicar un bineado a la WDLF teórica en intervalos pequeños de magnitud bolométrica (de 1 a 0.5 magnitudes) de la siguiente manera

$$
\langle n(L)\rangle_{\Delta M_{b o l}}=\frac{1}{\Delta M_{b o l}} \int_{l-0.5 \Delta M_{b o l}}^{l+0.5 \Delta M_{b o l}} n(L) d M_{b o l},
$$

donde $\Delta M_{b o l}$ representa el tamaño del bin de magnitud bolométrica.

Finalmente, Isern et al. (2008) mostró que la parte caliente de la WDLF es una herramienta poderosa para testear la existencia de partículas masivas débilmente interactuantes tales como el axión, debido a que esta función sólo es sensible a la tasa de enfriamiento de EB. Asimismo, tales autores mostraron que la forma de la WDLF podría ser afectada por axiones y que el cambio que estas partículas producen en la WDLF podría ser medido. Estas conclusiones junto a la forma teórica de la WDLF dada en la ec. (1.7) constituyen la base del estudio desarrollado en el capítulo 4. 


\subsection{Estrellas Cefeidas}

\subsubsection{Breve Reseña Histórica de las Cefeidas}

El estudio de las estrellas Cefeidas se ha venido desarrollando desde hace poco más de 200 años. La denominación de estrella Cefeida proviene de su arquetipo $\delta$ Cefeida cuya variabilidad fue descubierta por Goodricke (1786). A pesar de que la física presente en estas estrellas variables era desconocida, una gran cantidad de las mismas fueron catalogadas en esa época. Las Cefeidas son estrellas de masa intermedia, varias veces más masivas que el Sol, con períodos muy regulares que suelen estar comprendidos entre unas pocas horas a meses. Durante un ciclo de variabilidad estas estrellas pueden aumentar hasta cuatro veces su brillo. Además su curva de luz suele ser, en la mayoría de los casos, asimétrica con un rápido ascenso hacia la luminosidad máxima y un descenso más lento hacia la mínima.

Shapley (1914) determinó que la curva de luz de las estrellas Cefeidas no estaba regida por la binariedad sino por pulsaciones estelares de una única estrella. Asimismo, Baade (1926) propuso el método observacional para testear dichas pulsaciones. Estas técnicas serían luego mejoradas por Wesselink (1946), dando lugar a la teoría pulsacional. Hoy en día conocemos estas prescripciones como técnicas de Baade-Wesselink, las cuales permiten determinar, entre otras propiedades, el tamaño de las estrellas pulsantes.

En lo referente al estudio teórico de estas estrellas, Baker \& Kippenhahn (1962) mostraron que el fenómeno de variabilidad ocurre en la zona de ionización del helio mediante el uso de ecuaciones linealizadas independientes del tiempo para representar a las pulsaciones radiales. A partir del desarrollo computacional surgido en los años sesenta se descubrieron las anomalías en las masas, dando lugar a una discrepancia en las masas calculadas a través de cálculos evolutivos y pulsacionales.

Gracias a las nuevas técnicas de alta precisión, al uso de observaciones multibanda y al incremento de observaciones en infrarrojo, el estudio de estrellas Cefeidas ha mejorado notablemente en los últimos 40 años. Más precisamente, se pudo determinar con excelente precisión el punto cero y la pendiente de la relación Período-Luminosidad (la calibración de tal relación es esencial para determinar distancias extragalácticas). Gracias al telescopio espacial Hubble, las escalas de distancias obtenidas a partir de es- 
trellas Cefeidas pudieron ser extendidas hasta los cúmulos de galaxias Virgo y Fornax, ver Freedman et al. (2001).

\subsubsection{Características generales de las Cefeidas}

Las Cefeidas son estrellas variables y pueden ser clasificadas en dos tipos. Las de tipo I o Cefeidas Clásicas son estrellas jóvenes (de población I) y las de tipo II son estrellas viejas (de población II). Dado un período " $P$ " de variabilidad, las Cefeidas de tipo II son menos luminosas y masivas y por otra parte difieren en la relación Período-Luminosidad (P-L) con respecto a las Cefeidas de tipo I. El prototipo de estas estrellas variables es $\delta$ Cefeida aunque ésta fue la segunda en ser descubierta. En la actualidad hay más de 800 Cefeidas Clásicas en nuestra Galaxia y miles de Cefeidas extragalácticas, ver Szabados (2003) y Szabados (2010). De aquí en adelante nos referiremos a las Cefeidas de tipo I o Clásicas como "Cefeidas", ya que son el objeto de estudio de esta Tesis.

En términos generales, las Cefeidas son estrellas jóvenes (50-300 Myr), de masa intermedia (entre 4-10 $\mathrm{M}_{\odot}$ ), supergigantes amarillas de población I (tipo espectral F6-K2) cuyas pulsaciones radiales producen variaciones periódicas en el radio, temperatura y brillo superficial. En lo que respecta a períodos radiales de pulsación, éstos oscilan entre 1 y 200 días y son excitados a través del mecanismo $\kappa$ y $\gamma$, ver Gautschy (1997). En la Vía Láctea, el rango en períodos va desde 1 a 90 días mientras que en las nubes de Magallanes se han observado períodos superiores a los 200 días. Este tipo de variables pulsantes se encuentran ubicadas en una zona particular del diagrama de Hertzsprung Russell (HR) denominada "banda de inestabilidad", ver figura 1.4. Esta banda es una región estrecha casi vertical del diagrama que contiene distintos tipos de variables como RR Lyrae, Cefeidas, W Virginis y estrellas ZZ Ceti. La mayoría de las estrellas de masa intermedia ingresan a la banda de inestabilidad y se convierten en Cefeidas luego de salir de la secuencia principal. Es allí donde sufren inestabilidades que causan las pulsaciones, variación de tamaño y luminosidad.

A partir de una composición química $Z$ de tipo solar y sabiendo que estas estrellas

son pulsantes fundamentales, Bono et al. (2000) obtuvieron la siguiente relación entre el período $P$, la temperatura efectiva $T e f f$, la masa $M$ y la luminosidad $L$ 


$$
\log (P)=0.987-3.108 \log (\text { Teff })-0.767 \log \left(M / M_{\odot}\right)+0.942 \log \left(L / L_{\odot}\right)
$$

La relación anterior es fundamental para la determinación de distancias galácticas y extragalácticas a partir de estas estrellas.

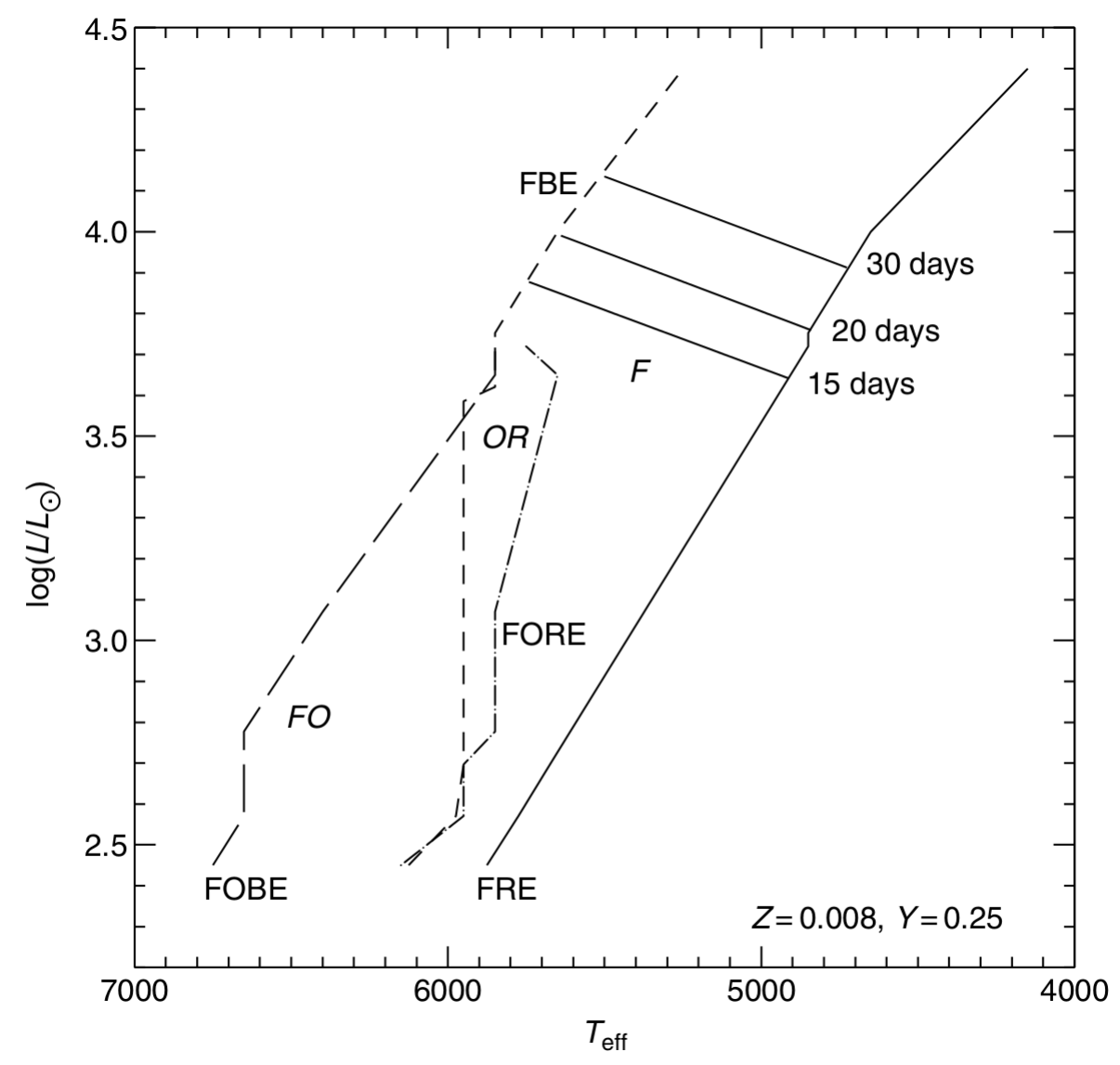

Figura 1.4: Ubicación de la banda de inestabilidad de las Cefeidas en el diagrama H-R. Las etiquetas indican la zona de inestabilidad de los modos pulsacionales. F indica el modo fundamental de pulsación, FO el primer fundamental, FOBE el límite azul para la región pulsacional, FRE el límite rojo de la misma, FBE el límite azul para el fundamental y FORE indica el límite rojo para el primer fundamental. Se muestran además las líneas de período constante. Figura extraída de Salaris \& Cassisi (2006).

\subsubsection{Evolución Estelar de las Cefeidas}

Los progenitores de estas estrellas son más masivos que el Sol y entran en la categoría de estrellas de masa intermedia. Ahora bien, existen dos maneras de determinar la masa de estas estrellas variables, una es a partir de consideraciones evolutivas y la otra 
es a partir de cálculos pulsacionales, ver Caputo et al. (2005). Las masas evolutivas se estiman utilizando la comparación entre isocronas y observaciones en el diagrama Color-Magnitud. A diferencia de ello, las masas pulsacionales se determinan utilizando la relación Período-Masa-Radio de Cefeidas Galácticas. Si bien la diferencia entre las masas obtenidas por ambos métodos teóricos ha ido disminuyendo, todavía existe en la actualidad una discrepancia del $10 \%$ al $20 \%$. Sin embargo, la inclusión de pérdidas de masas en los modelos evolutivos podría reducir dicha discrepancia (Neilson et al. $(2012))$.

La figura 1.5 muestra la localización en el diagrama Color-Magnitud de Cefeidas Clásicas en la Nube Mayor de Magallanes. De dicha figura se observa claramente que las Cefeidas de distintos modos pulsacionales (puntos a color) ocupan, como era de esperarse, la banda de inestabilidad entre las estrellas de secuencia principal y las estrellas gigantes rojas (puntos en negro).

Luego del agotamiento del $\mathrm{H}$ en el núcleo, los progenitores se mueven desde la secuencia principal hasta la rama de las gigantes rojas cruzando por primera vez la banda de inestabilidad. Este primer cruce es rápido de manera que es esperable que relativamente pocas Cefeidas sean observadas durante el mismo. Estas estrellas entran por segunda vez en la banda de inestabilidad luego del comienzo de la quema de He en el núcleo. En efecto, dichas estrellas evolucionan siguiendo loops que las llevan desde la región de las gigantes rojas al azul atravesando la banda de inestabilidad, ver figura 1.6. Una vez que las estrellas alcanzan el punto más azul de tales loops, evolucionan hacia el rojo nuevamente y pueden cruzar por tercera la banda. En general, el segundo y el tercer cruce toman más tiempo que el primero por lo que la estrella tiene gran posibilidad de ser observada en las correspondientes etapas evolutivas. Los mencionado loops, conocido usualmente como "blue loops", son necesarios para explicar la existencia de las gigantes amarillas, las supergigantes y las $\delta$ Cefeidas. Asimismo, la luminosidad de los blue loops depende de la masa estelar inicial, siendo más luminosa para masas más altas (Caputo et al. (2005)). Tanto la extensión y duración de los blue loops son sensibles a los detalles de los cálculos de evolución estelar. Mientras que algunos detalles tales como la composición química y la rotación involucran propiedades intrínsecas de las estrellas, otros, tales como el tratamiento del overshooting convectivo en el núcleo y en la envoltura, 


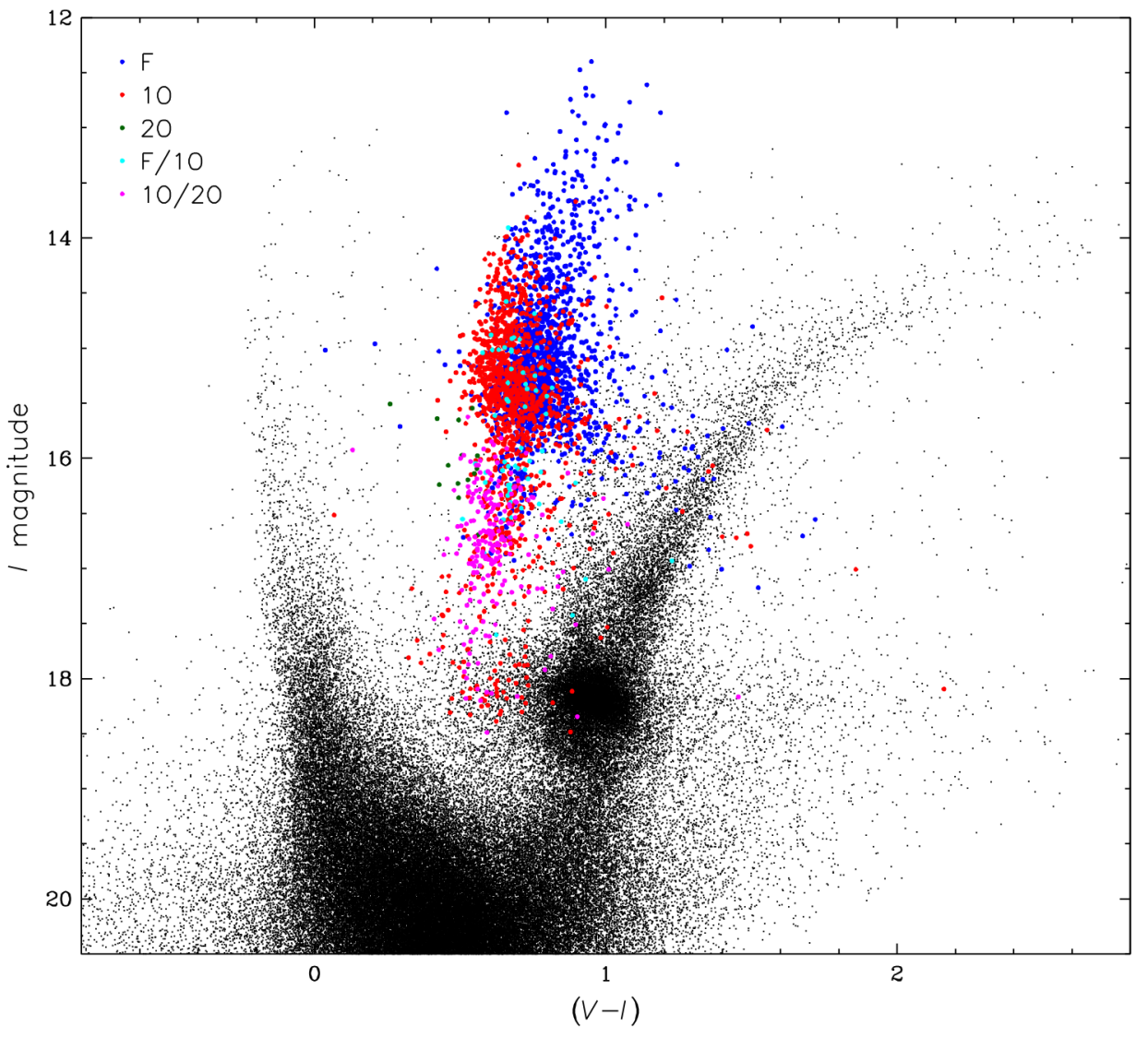

Figura 1.5: Diagrama Color-Magnitud para Cefeidas Clásicas en la Nube Mayor de Magallanes. Las estrellas de fondo (puntos negros) pertenecen al subcampo LMC100.1. Los distintos colores representan Cefeidas que están pulsando en un modo particular. Los puntos azul oscuro representan los modos fundamentales de pulsación, los rojos el primer armónico y los verdes el segundo armónico. Los puntos azul claro representan modos de pulsación mixtos. Datos extraídos del relevamiento OGLE, ver Soszyński et al. (2008).

dependen del código numérico utilizado para computar la evolución de la estrella. Una conclusión aceptada por todos los cálculos que involucran formación de blue loops es que éstos se activan cuando la quema en capa del $\mathrm{H}$ se intensifica durante la quema de He en el núcleo (Halabi et al. (2012)). Dicha intensificación depende fuertemente de la forma del perfil de $\mathrm{H}$ creado después del primer dredge up (FDUP) en la rama asintótica de las gigantes. 


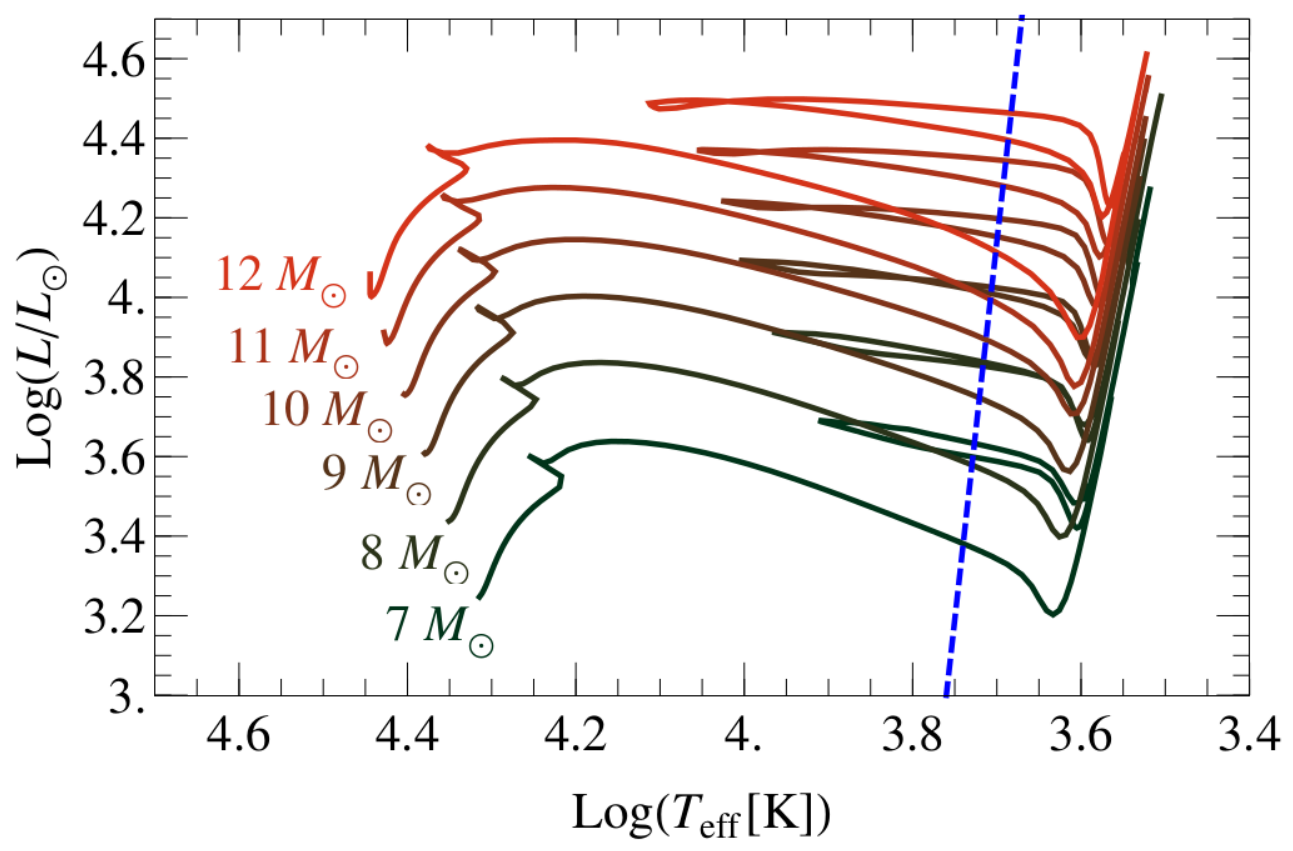

Figura 1.6: Evolución en el diagrama HR para estrellas con masas entre $7 M_{\odot}-12 M_{\odot}$. La línea puntea representa la banda de inestabilidad de las Cefeidas, tomado de Friedland et al. (2013). 


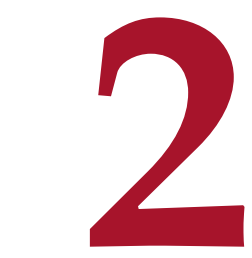

Axiones

\subsection{El Problema fuerte de Carga-Paridad}

El término Carga-Paridad (CP) es una nomenclatura técnica utilizada para la simetría discreta que resulta del producto de dos simetrías independientes: la conjugación de carga (C), que implica el intercambio de una dada partícula por la correspondiente antipartícula, y la paridad (P), la cual involucra una inversión de las coordenadas espaciales. La evidencia experimental indica que CP es conservada en las interacciones fuertes y en las electromagnéticas pero es violada por las interacciones débiles. A su vez, en el denominado Modelo Estándar de las partículas elementales, cualquier ruptura de la simetría CP en las interacciones débiles puede inducir una ruptura de simetría en las interacciones fuertes, ver Quinn (2001).

La simetría partícula-antipartícula se manifiesta cuando las interacciones de gauge y electrodébiles conservan CP. Por el contrario, la simetría no se ve respetada por los acoplamientos complejos de Yukawa al campo de Higgs. Esto trae como consecuencia la aparición de una transformación global de fase, que se adiciona al lagrangiano de QCD y toma la siguiente forma

$$
\mathcal{L}_{\Theta}=\Theta \frac{\alpha_{s}}{8 \pi} G \tilde{G}
$$


donde $\alpha_{s} \simeq g_{s}^{2} / 4 \pi$ es la constante de estructura fina de las interacciones fuertes y $g_{s}$ representa la constante de acoplamiento de Yukawa, ver Halzen \& Martin (1984). G $\tilde{G}$ representa el producto $G_{b}^{\mu \nu} \tilde{G}_{b \mu \nu}$ donde $G_{b}^{\mu \nu}$ es el tensor de campo de color y $\tilde{G}_{b \mu \nu}=$ $\frac{1}{2} \epsilon_{\mu \nu \rho \sigma} G_{b}^{\rho \sigma}$ su dual, donde $b$ simboliza los grados de libertad de color. El ángulo $\Theta$ de la ec. (2.1) puede escribirse como

$$
\Theta=\theta+\arg \operatorname{det} M_{q}
$$

El parámetro $\theta$ define el estado fundamental de QCD, también llamado estado de vacío. La matriz $M_{q}$ presente en el segundo término de la ec. (2.2) es la matriz compleja de masa para los quarks, la cual puede ser llevada, mediante una transformación, a una matriz diagonal con todos sus elementos reales. Este procedimiento agrega el término $\arg \operatorname{det} M_{q}$ al lagrangiano de QCD debido a la transformación global mencionada anteriormente.

$\mathrm{Al}$ actuar en forma simultánea las transformaciones de carga y paridad sobre $\mathcal{L}_{\Theta}$, este cambia de signo violándose así la invarianza ante $\mathrm{CP}$ de QCD, lo que da lugar a un momento dipolar eléctrico del neutrón, $d_{n}$, no nulo. Ahora bien, la predicción teórica para los momentos dipolares eléctricos basada en $\mathcal{L}_{\Theta}$ y asumiendo al parámetro $\Theta$ como de $\mathcal{O}(1)$ resulta al menos $10^{10}$ veces mayor que los valores obtenidos experimentalmente. Es preciso mencionar que el momento dipolar eléctrico del neutrón, $d_{n}$, no ha sido observado. Los valores experimentales actuales ponen restricciones sobre $\left|d_{n}\right|$ (ver tabla 2.1), los cuales acotan al parámetro $\Theta$ de la siguiente manera: $\Theta<4 \times 10^{-10}$. El momento dipolar eléctrico del neutrón está dado aproximadamente por:

$$
\frac{e}{m_{n}} \Theta \frac{m_{u} m_{d}}{m_{u}+m_{d}} \frac{1}{\Lambda_{Q C D}}
$$

donde $\Lambda_{Q C D}$ es la escala de QCD y $m_{n}$ la masa del neutrón. Esta discrepancia es lo que se conoce como Problema Fuerte de C-P. 


\begin{tabular}{ccc}
\hline \hline Fermión & Momento Magnético & Momento Eléctrico \\
\hline Protón & $2.792847356 \mu_{N}$ & $\leq 0.54 \times 10^{-23} \mathrm{ecm}$ \\
Neutrón & $-1.9130427 \mu_{N}$ & $\leq 0.29 \times 10^{-25} \mathrm{ecm}$ \\
Electrón & $1159.65218076 \times 10^{-6}$ & $\leq 0.87 \times 10^{-28} \mathrm{ecm}$ \\
Neutrino & $\leq 0.29 \times 10^{-10} \mu_{B}$ & $\leq 6000 \times 10^{-26} \mathrm{ecm}$ \\
\hline \hline
\end{tabular}

Tabla 2.1: Valores y cotas para los momentos dipolares eléctricos y magnéticos extraídos de Olive et al. (2014), "The Review of Particle Physics (2015)". $\mu_{B}$ es el magnetón de Bohr y $\mu_{N}$ es el magnetón nuclear.

\subsection{El modelo de Peccei-Quinn}

Peccei \& Quinn (1977b) (PQ) propusieron una solución fundamental al problema fuerte de CP mediante la incorporación de una nueva simetría global $U(1)_{P Q}$ al Modelo Estándar. Dicha simetría es rota espontáneamente, lo que da lugar a la aparición de un bosón de Nambu-Goldstone. Dicho bosón no posee espín, carga ni masa. Sin embargo, si la simetría global es aproximada, la partícula resultante de la ruptura espontánea de simetría tiene masa y se conoce como "pseudo-bosón de Goldstone". Esta simetría adicional trae consigo un campo, que es representado a partir de un bosón de gauge denominado "axión".

El concepto básico en el mecanismo de Peccei-Quinn subyace en que $\Theta$ sea un campo dinámicamente variable con un valor mínimo en cero y que no represente un parámetro libre o una constante de acoplamiento de la teoría. Para $\Theta=0$, PQ introdujeron la simetría $U(1)_{P Q}$ anteriormente mencionada. En este sentido, el campo propuesto adiciona un nuevo término $L_{\text {axion }}$ al lagrangiano de QCD de la siguiente manera:

$$
\mathcal{L}_{Q C D}=\mathcal{L}_{\text {pert }}+\mathcal{L}_{\Theta}+\mathcal{L}_{\text {axion }}
$$

donde $\mathcal{L}_{\text {pert }}$ representa la perturbación al lagrangiano original, $\mathcal{L}_{\Theta}$ toma la expresión de la ec.(2.1) y el último término se puede escribir como 


$$
\mathcal{L}_{\text {axion }}=\mathcal{L}_{\text {cin }}+\mathcal{L}_{\text {int }}-\mathcal{V}_{\text {eff }} .
$$

El primer término de la ec.(2.5) representa la energía cinética,

$$
\mathcal{L}_{\text {cin }}=-\frac{1}{2}\left(\partial_{\mu} a\right)\left(\partial^{\mu} a\right),
$$

el segundo tiene en cuenta la posible interacción con otros axiones y el último contabiliza el potencial efectivo al interactuar con gluones. Para calcular el valor de expectación del campo del axión, es necesario buscar los extremos del potencial. La minimización del potencial da como resultado la siguiente expresión:

$$
\langle a\rangle=-\frac{f_{a}}{C_{a}} \Theta,
$$

donde $C_{a}$ es un parámetro que depende del modelo, $a$ representa el campo del axión y $f_{a}$ es la escala de ruptura de simetría de PQ.

De la expresión (2.5), se puede derivar que el campo del axión debe ser impar bajo la transformación $\mathrm{CP}$ a fin de mantener la invarianza ante dicha simetría de $\mathcal{L}_{\text {axion }}$. Esto implica que el axión tiene paridad negativa y, por lo tanto, es un bosón pseudoescalar.

Aunque los axiones fueron construidos con la propiedad de ser no masivos, estas partículas adquieren una masa efectiva mediante la interacción con otros gluones. En efecto, a través de su interacción con los gluones, el axión puede efectuar transiciones al estado $q \bar{q}$ (quark-antiquark) y, por lo tanto, a piones neutros, ver Fig.(2.1), produciéndose una mezcla.

Existe por tanto una relación entre la masa del axión y la del pión dada por

$$
m_{a} f_{a} \simeq m_{\pi} f_{\pi},
$$




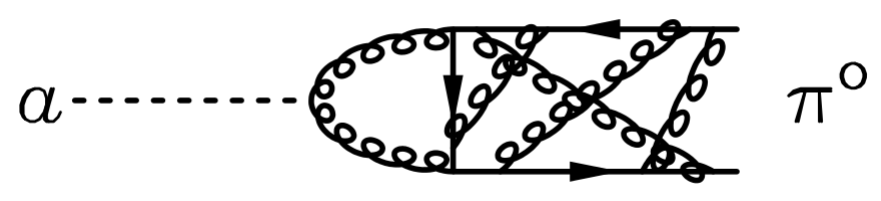

Figura 2.1: Diagrama que representa la mezcla del axión con el pión neutro. Las lineas curvas representan a los gluones y las rectas a los quarks, ver Raffelt (1996).

donde $m_{\pi}=135 \mathrm{MeV}$ es la masa del pión y $f_{\pi} \simeq 93 \mathrm{MeV}$ su constante de decaimiento. Además, debido a la mezcla con piones neutros, los axiones también se acoplarán a fotones y nucleones, aunque con una intensidad reducida en un factor $f_{\pi} / f_{a}$.

\subsection{Modelos fenomenológicos De AXiones}

\subsubsection{El axión de Peccei-Quinn-Weinberg-Wilczeck}

Desde la perspectiva de $\mathrm{PQ}$, el axión propuesto originalmente se fundamentó en la idea de que la ruptura espontánea de simetría era del orden de la escala débil, $f_{\text {weak }}=250 \mathrm{GeV}$, lo que a su vez implica una masa del axión mayor a $150 \mathrm{KeV}$, valor que sería excluido rápidamente. La denominación para esta partícula fue la de "axión-PQWW" debido a sus autores Peccei, Quinn, Weinberg y Wilczek. Asimismo, el nombre utilizado para dicho axión pesado fue "axión visible", en contraposición al "axión invisible" o muy liviano definido con posterioridad.

PQ adoptaron un modelo de interacción que incluía la violación débil de CP y donde el acoplamiento de los axiones a leptones y quarks pesados puede ser obtenido de manera sencilla. La intensidad de acoplamiento de los axiones con fermiones puede ser obtenida a través del álgebra de corriente. Para ello, se necesita introducir dos dobletes de Higgs que tomen valores de expectación de vacío $\lambda_{1}$ y $\lambda_{2}$. Además, al cociente es denotado como $x$ y se lo elije positivo, de manera que $x>1$. La escala de ruptura simetría viene entonces dada por

$$
f_{a}=\sqrt{\lambda_{1}^{2}+\lambda_{2}^{2}}=\left(\sqrt{2} G_{F}\right)^{-1 / 2}=f_{\text {weak }} \simeq 250 \mathrm{GeV},
$$


donde $G_{F}$ denota la constante de acoplamiento de Fermi. Utilizando métodos del álgebra de corriente estándar, Bardeen \& Tye (1978) obtuvieron para la masa del axión $m_{a}$ :

$$
m_{a}=N\left(\frac{1}{x}+x\right) \frac{\sqrt{z}}{1+z} \frac{f_{\pi} m_{\pi}}{f_{a}}
$$

donde $N$ denota el número de generaciones de quarks, $z$ el cociente entre la masa del quark "u" y la del "d", $m_{\pi}=135 \mathrm{MeV}$ es la masa del pión y $f_{\pi}=93 \mathrm{MeV}$ la correspondiente constante de decaimiento. Por tanto la masa del axión viene dada como

$$
m_{a} \simeq 25 N\left(\frac{1}{x}+x\right) \mathrm{KeV} .
$$

Como $N=3$ y $x>1$, se obtiene de la ec. (2.11) que $m_{a}>150 \mathrm{KeV}$.

\subsubsection{Modelos Invisibles de Axiones: KSVZ y DFSZ}

Como los axiones visibles fueron descartados experimentalmente, Wise et al. (1981) introdujeron el concepto de axión invisible. Debido a que la escala de simetría $f_{a}$ es en principio arbitraria, estos autores escogieron un valor por encima de la escala de ruptura de simetría de la teoría electrodébil a fin de que el acoplamiento resultase mucho más débil y, por ende, el axión adquiere una masa mucho más pequeña que la esperada en el caso del axión de PQWW. Por este motivo, se denominó a los axiones correspondientes a este modelo como "axiones invisibles".

El modelo más sencillo de axiones invisibles es el de Kim-Shifman-Vainshtein-Zakharov (KSVZ), ver Kim (1979) y Shifman et al. (1980). En el mismo se introduce un quark pesado exótico "Q", con carga de PQ, mientras que se asume que los quarks ordinarios, leptones y campos de Higgs no son cargados respecto de la simetría de PQ. El segundo modelo, debido a Dine-Fischler-Srednicki-Zhitnitsky (DFSZ), ver Dine et al. (1981) y Zhitnitsky (1980), se introdujo poco tiempo después y no introduce quarks exóticos. En cambio, dos dobletes de Higgs se incluyeron de manera complementaria, para que los mismos y los quarks convencionales no presentaran carga de PQ. 
Una propiedad genérica para ambos modelos es que la interacción de axiones con dos fotones viene dada por:

$$
\mathcal{L}_{a \gamma \gamma}=\frac{g_{a \gamma \gamma}}{4} F_{\mu \nu} \tilde{F}^{\mu \nu} a=-g_{a \gamma \gamma} \bar{E} \cdot \bar{B} a
$$

donde $F$ es el tensor de campo electromagnético, $\tilde{F}$ su dual, $\bar{E}$ y $\bar{B}$ los campos eléctricos y magnéticos, respectivamente, y a representa el campo del axión. La constante de acoplamiento $g_{a \gamma \gamma}$ que aparece en la ec. (2.12) toma la siguiente forma:

$$
g_{a \gamma \gamma}=\frac{\alpha}{2 \pi f_{a}}\left(\frac{E}{N}-\frac{2}{3} \frac{4+z}{1+z}\right)=\frac{\alpha}{2 \pi}\left(\frac{E}{N}-\frac{2}{3} \frac{4+z}{1+z}\right) \frac{1+z}{z^{1 / 2}} \frac{m_{a}}{m_{\pi} f_{\pi}} .
$$

En la ec. (2.13), E y $N$ son las anomalías de color y electromagnéticas, respectivamente, para la corriente axial asociada al campo del axión. Es preciso mencionar que en física cuántica una anomalía se define como la ruptura de simetría del lagrangiano de una teoría clásica. En el caso del modelo KSVZ, el cociente de $E$ con $N$ resulta ser nulo, mientras que para modelos unificados como el DFSZ este cociente toma el valor de 8/3. En el caso más general, $E / N$ no se conoce y, por tanto, si se fija $f_{a}$, existe un amplio rango de valores para la constante de acoplamiento $g_{a \gamma \gamma}$, ver Cheng et al. (1995). Asumiendo que no existen cancelaciones antinaturales, la escala de $g_{a \gamma \gamma}$ viene dada por la interacción del pión. De esta forma, tomando los factores que son modelo dependientes del orden de la unidad, se define a partir de la ec. (2.13 ) la "línea axiónica" en el plano $m_{a^{-}} g_{a \gamma \gamma}$

La tasa de decaimiento, la cual es un indicador de la velocidad con que una población reduce su tamaño, del axión a dos fotones que se sigue de la interacción dada en la ec. (2.12) con la constante de acoplamiento de la ec. (2.13) es

$$
\Gamma_{a \rightarrow \gamma \gamma}=\frac{g_{a \gamma \gamma} m_{a}^{3}}{64 \pi}=\frac{\alpha^{2}}{256 \pi^{3}}\left[\left(\frac{E}{N}-\frac{2}{3} \frac{4+z}{1+z}\right)\right]^{2} \frac{m_{a}^{5}}{m_{\pi}^{2} f_{\pi}^{2}}=1.1 \times 10^{-24} s^{-1}\left(\frac{m_{a}}{e V}\right),
$$


donde la primera expresión es válida para toda partícula pseudoescalar, la segunda se aplica específicamente para axiones y, finalmente, la expresión numérica corresponde a $z=0.56$ y axiones $\operatorname{KSVZ}(E / N=0)$.

La interacción a fermiones "j" tiene una estructura derivativa, esto significa que es invariante bajo la transformación $a \rightarrow a+a_{o}$, debido a que el axión es un bosón de Nambu-Goldstone entonces el lagrangiano puede escribirse como

$$
\mathcal{L}_{a j j}=\frac{C_{j}}{2 f_{a}} \bar{\psi}_{j} \gamma^{\mu} \gamma_{5} \psi_{j} \partial_{\mu} a=-i \frac{C_{j} m_{j}}{f_{a}} \bar{\psi}_{j} \gamma_{5} \psi_{j} a
$$

En la última expresión, $\psi_{j}$ es el campo del fermión, $m_{j}$ su masa y $C_{j}$ un coeficiente numérico que depende del modelado. El cociente definido como $g_{a j j}=C_{j} m_{j} / f_{a}$ toma el rol de los acoplamientos de Yukawa y $\alpha_{a j j}=g_{a j j}^{2} / 4 \pi$ es el análogo a la constante de estructura fina.

En los modelos hadrónicos, como el KSVZ, los axiones no se acoplan a quarks y leptones a orden árbol, mientras que en el modelo DFSZ se tiene

$$
C_{e}=\frac{\cos ^{2} \beta}{3}
$$

donde $\cos \beta$ representa el cociente de dos valores de expectación de vacío para el campo de Higgs en este modelo.

Por completitud, se incluye la interacción con nucleones, donde el acoplamiento adimensional, $C_{n, p}$, se escribe en términos de los elementos de matriz de la corriente axial vectorial como sigue

$$
\begin{aligned}
& C_{p}=\left(C_{u}-\eta\right) \Delta u+\left(C_{d}-\eta z\right) \Delta d+\left(C_{s}-\eta w\right) \Delta s, \\
& C_{n}=\left(C_{u}-\eta\right) \Delta d+\left(C_{d}-\eta z\right) \Delta u+\left(C_{s}-\eta w\right) \Delta s .
\end{aligned}
$$

Aquí $\eta=(1+z+w)^{-1}$, con $z=m_{u} / m_{d}$ y $w=m_{u} / m_{s}$; la cantidad $\Delta q$ representa el acoplamiento de la corriente axial vectorial con el protón. 
Emisión de AXIONes KSVZ y EL PRoceso PRimakoff en estrellas Cefeidas

El acoplamiento de dos fotones a piones o a otros pseudoescalares, permite la conversión $a-\gamma$ en un campo eléctrico o magnético externo, en virtud de la amplitud que se muestra en la figura 2.2. Primakoff (1951) propuso este proceso por primera vez, para estudiar el acoplamiento $\pi^{0}-\gamma$, que experimentalmente es difícil de medir en los decaimientos libres $\pi^{0} \rightarrow 2 \gamma$. En las estrellas de masa intermedia como lo son las Cefeidas, este proceso permite la producción de pseudoescalares de baja masa en los campos eléctricos de los núcleos y electrones, ver Dicus et al. (1978). El proceso Primakoff se da

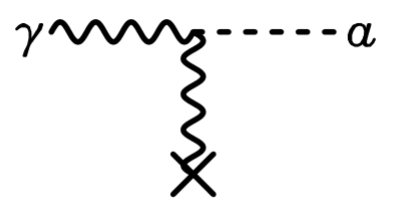

Figura 2.2: Conversión Primakoff entre axiones con fotones en un campo electromagnético externo, ver Raffelt (1996).

bajo condiciones no relativistas donde $T<<m_{e}$, por lo tanto los electrones y núcleos pueden ser tratados como partículas pesadas en comparación a las energías típicas para los fotones. De esta manera, si se ignoran los efectos de retroceso, se encuentra para la sección eficaz diferencial:

$$
\frac{d \sigma_{\gamma \rightarrow a}}{d \Omega}=\frac{g_{a \gamma}^{2} Z^{2} \alpha}{8 \pi} \frac{\left|\bar{k}_{\gamma} \times \bar{k}_{a}\right|^{2}}{q^{4}}
$$

donde $\bar{q}=\bar{k}_{\gamma}-\bar{k}_{a}$ es el momento transferido, donde la carga del blanco resulta ser $Z e$.

La tasa de transición, expresada en unidades naturales ${ }^{*}$, para un fotón de energía $E$ en un axión con la misma energía, ver ec. (5.8) de Raffelt (1996), viene dada por:

$$
\Gamma_{\gamma \rightarrow a}=\frac{g_{a \gamma \gamma}^{2} T k_{s}^{2}}{32 \pi}\left[\left(1+\frac{k_{s}^{2}}{4 w^{2}}\right) \ln \left(1+\frac{4 w^{2}}{k_{s}^{2}}\right)-1\right],
$$

\footnotetext{
${ }^{*}$ En unidades naturales se considera $c=\hbar=1$
} 
donde $\kappa_{s}$ es el número de onda de apantallamiento de Debye-Huckel y está definido de la siguiente manera:

$$
\kappa_{s}^{2}=\frac{4 \pi \alpha}{T}\left(n_{e}+\sum_{\text {nucleos }} Z_{j}^{2} n_{j}\right)
$$

siendo $n_{e}$ la densidad electrónica y $n_{j}$ la densidad del ion $j$-ésimo de carga $Z_{j}$. Asimismo, la emisión de axiones $\epsilon_{A}$ por efecto Primakoff en un medio no degenerado, presente en el trabajo de Carosi et al. (2013), es:

$$
\epsilon_{A}=Z\left(\xi^{2}\right) \frac{g_{a \gamma \gamma}^{2}}{4 \pi^{2}} \frac{T^{7}}{\rho} \simeq 28 \frac{e r g}{g . s} Z\left(\xi^{2}\right) g_{10}^{2} T_{8}^{7} \rho_{4}^{-1}
$$

donde se definen las variables como sigue: $\rho_{4}=\rho /\left(10^{4} \mathrm{~g} / \mathrm{cm}^{3}\right), T_{8}=T / 10^{8} \mathrm{~K}, g_{10}=$ $g_{a \gamma \gamma} 10^{10} \mathrm{GeV}$ y $\xi^{2}=\left(\kappa_{s} / 2 T\right)^{2}$.

Es preciso mencionar que al considerar la emisión de axiones KSVZ en el código de evolución estelar LPCODE, se ha utilizado la parametrización de $Z\left(\xi^{2}\right)$ incluida en el trabajo de Friedland et al. (2013), donde se propone la siguiente función:

$$
Z\left(\xi^{2}\right) \simeq\left(\frac{1.037 \xi^{2}}{1.01+\xi^{2} / 5.4}+\frac{1.037 \xi^{2}}{44+0.628 \xi^{2}}\right) \ln \left(3.85+\frac{3.99}{\xi^{2}}\right)
$$

\section{EMisión de AXiones DFSZ en estrellas EnAnas BlancAs}

Como se dijo anteriormente, uno de los modelos más discutidos en la literatura es el modelo DFSZ. En contraste con el modelo KSVZ, no se incluyen quarks pesados exóticos sino sólo fermiones que transportan carga de PQ. Desde una perspectiva práctica, la principal diferencia con el modelo KSVZ, es que en el modelo DFSZ los axiones se acoplan a leptones cargados además de a nucleones y a fotones.

A continuación, se describirá la expresión adoptada para la emisividad de axiones DFSZ, en el contexto de las estrellas EBs. Por completitud, se incluyen en esta emisión los procesos Compton y Bremmsstrahlung, aunque solamente éste último proceso es de importancia en las EBs. Además, se tienen en cuenta las correlaciones iónicas fuertes 
$(\Gamma>1)^{* *}$ y débiles $(\Gamma<1)$, tanto para el régimen fuertemente degenerado como para el no degenerado.

En el caso de la emisión de axiones por Bremsstrahlung bajo condiciones degeneradas $\left(\epsilon_{B D}\right)$, se adoptan las prescripciones de Nakagawa et al. $(1987,1988)$ para un plasma en un régimen fuertemente acoplado y Raffelt \& Weiss (1995) para un plasma débilmente acoplado. Se tiene entonces que:

$$
\epsilon_{B D}=10.85 \alpha_{26} T_{8}^{4} \sum_{j}^{N_{\text {isot }}} \frac{X_{j} Z_{j}^{2}}{A_{j}} \times F_{j}
$$

donde $A_{j}$ representa el número másico, $Z_{j}$ el número atómico y $X_{j}$ la fracción numérica de la especie atómica $j$-ésima. Además, la expresión para $F_{j}$ se encuentra en el trabajo de Nakagawa et al. $(1987,1988)$ para $\Gamma>1$ y en el caso de $\Gamma<1$ está dado por $F_{j}=F$ donde

$$
F=\frac{2}{3} \ln \left(\frac{2+\kappa^{2}}{\kappa^{2}}\right)+\left[\frac{2+5 \kappa^{2}}{15} \ln \left(\frac{2+\kappa^{2}}{\kappa^{2}}\right)-\frac{2}{3}\right] \times \beta_{F}{ }^{2}
$$

con $\beta_{F}$ y $\kappa$ dados por

$$
\begin{gathered}
\kappa^{2}=\frac{2 \pi \alpha \hbar^{3} c}{m_{u} k} \frac{\rho}{T} \sum_{j} \frac{X_{j} Z_{j}^{2}}{A_{j}} \frac{1}{p_{F}^{2}}, \\
\beta_{F}{ }^{2}=\frac{p_{F}{ }^{2}}{m_{e}{ }^{2} c^{2}+p_{F}^{2}}, p_{F}{ }^{2}=\hbar\left(\frac{3 \pi^{2} \rho}{\mu_{e} m_{u}}\right)^{1 / 3} .
\end{gathered}
$$

Para el régimen no degenerado $\left(\epsilon_{B N D}\right)$, el Bremsstrahlung fue obtenido de las expresiones presentadas en Raffelt (1996) como:

\footnotetext{
${ }^{* *}$ Donde la constante de acoplamiento iónica, $\Gamma$, para un plasma multicomponente se define siguiendo Segretain et al. (1994) como

$$
\Gamma=2.275 \times 10^{5} \frac{\left(\rho Y_{e}\right)^{1 / 3}}{T} \sum_{i}\left(n_{i} / n_{\text {ions }}\right) Z_{i}^{5 / 3},
$$

donde la suma se extiende sobre todas las especies nucleares consideradas.
} 
$\epsilon_{B N D}=5.924 \times 10^{-4} \alpha_{26} \frac{T_{8}^{5 / 2} \rho}{\mu_{e}} \sum_{j=1}^{N_{\text {isot }}}\left[\frac{X_{j}}{A_{j}}\right] \times\left[Z_{j}^{2}\left(1-\frac{5}{8} \frac{\kappa^{2} \hbar^{2}}{m_{e} T k}\right)+\frac{Z_{j}}{\sqrt{2}}\left(1-\frac{5}{4} \frac{\kappa^{2} \hbar^{2}}{m_{e} T k}\right)\right]$.

En esta expresión $\epsilon_{B N D}$ tiene unidades $\left(\operatorname{erg~g}^{-1} \mathrm{~s}^{-1}\right)$ y la escala de apantallamiento resulta dada por:

$$
\kappa^{2}=\frac{4 \pi \alpha \hbar c}{k T} \hat{n}, \hat{n} \simeq n_{e}+\sum_{j}^{N_{\text {isot }}} Z_{j}^{2} n_{j}
$$

En el caso de la emisión por procesos Compton se siguieron las prescripciones de Raffelt \& Weiss (1995), tomando en cuenta los bloqueos de Pauli bajo condiciones degeneradas e incluyendo correcciones relativistas:

$$
\epsilon_{\text {compton }}=33 \alpha_{26} Y_{e} T_{8}^{6} F_{c} \operatorname{erg~g}^{-1} \mathrm{~s}^{-1}
$$

donde $F_{c}=\left(1+F_{\text {comp.deg. }}{ }^{-2}\right)^{-1 / 2}$, con $F_{\text {comp.deg. }}=4.96 \times 10^{-6} \mu_{e}{ }^{2 / 3} T \rho^{-2 / 3}$, ver Raffelt $\&$ Weiss $(1995)$. Finalmente, la emisión total $\left(\epsilon_{\text {axion }}\right)$ es:

$$
\epsilon_{\text {axion }}=\epsilon_{\text {Brem }}+\epsilon_{\text {compton }}
$$

donde la emisión por Bremsstrahlung es obtenida de las ecs. (2.24) y (2.28) a través de:

$$
\epsilon_{\text {Brem }}=\left(\frac{1}{\epsilon_{\mathrm{BND}}}+\frac{1}{\epsilon_{\mathrm{BD}}}\right)^{-1}
$$

\subsection{IMPLEMENTACión DE RUTiNAS PARA LA EMISión DE AXIONES EN EL CÓdigo DE EVOLUCIÓN ESTELAR LPCODE.}

Se han incluido en el código de evolución estelar LPCODE, ver Apéndice A, dos rutinas que calculan las pérdidas anómalas de energía debidas a la emisión de axiones del 
tipo KSVZ y DFSZ. Estas rutinas permiten obtener la correspondiente tasa de pérdida de energía para cada tipo de acoplamiento, la cual es incorporada de manera autoconsistente en el cálculo de la ecuación de la luminosidad. A continuación se describen brevemente ambas rutinas.

\subsubsection{SUBRUTINA AXIONALL_KSVZ}

Esta subrutina desarrollada específicamente para la obtención de los resultados de esta Tesis calcula la emisión de axiones por efecto Primakoff, ver ec. (2.22), haciendo uso de las expresiones (2.21) y (2.23) para el número de onda de apantallamiento de Debye-Huckel, $\kappa_{s}$, y para la función $Z\left(\xi^{2}\right)$, respectivamente.

Las variables de entrada de la subrutina en términos de la nomenclatura usada en el código son las siguientes:

$$
\begin{aligned}
\text { rho } & =\text { densidad } \\
\text { rhop } & =\text { derivada de la densidad con respecto a la presión a temperatura constante, } \\
\text { rhot } & =\text { derivada de la densidad con respecto a la temperatura a presión constante, } \\
\text { temp } & =\text { temperatura, } \\
\text { ue } & =\text { inversa del número electrónico molar, }
\end{aligned}
$$

mientras que las variables de salida de la misma son:

$$
\begin{aligned}
\text { eaxion } & =\text { emisión de axiones } \epsilon_{A} \text { calculada en }(\mathrm{erg} / \mathrm{gs}) \\
\text { eaxionT } & =\text { derivada de } \epsilon_{A} \text { con respecto a la temperatura a presión constante, } \\
\text { eaxionP } & =\text { derivada de } \epsilon_{A} \text { con respecto a la presión a temperatura constante. }
\end{aligned}
$$

La emisión de axiones del tipo KSVZ y sus derivadas se determinan de forma procedimental llevando a cabo los siguientes pasos dentro de la subrutina:

- Cálculo del número de onda de apantallamiento de Debye-Huckel, ver ec. (2.21).

- Obtención de $Z\left(\xi^{2}\right)$ siguiendo la parametrización dada en la ec. (2.23), la cual fue propuesta en el trabajo de Friedland et al. (2013). 
- Por último, a partir de los pasos previos, se determina $\epsilon_{A}$ y sus respectivas derivadas. Cabe señalar que todas las cantidades programadas en esta subrutina se encuentran en el sistema cegesimal o c.g.s.

\subsubsection{SUbRUtinA AXIONALL_DFsZ}

Esta subrutina incluye varias rutinas que calculan la pérdida de energía por axiones del tipo DFSZ en distintos regímenes, para más detalle ver sección 4.2.1 de Miller Bertolami (2009). Es preciso mencionar que en esta subrutina se incluyen tanto procesos de emisión Compton como Bremmstrahlung, aunque en esta Tesis solo el último cobra relevancia en los cálculos referentes a las EBs. Para esta subrutina, las variables de entrada y salida son las mismas que en la subrutina axionall_ksvz, salvo que aquí se agrega como entrada adicional la masa de la partícula hipotética denominada en el LPCODE dm_axion.

La emisión de axiones del tipo DFSZ y sus derivadas se determinan procedimentalmente a través del siguiente esquema:

- Determinación de la constante de acoplamiento iónica $\Gamma$ para el gas multicomponente a través de la expresión:

$$
\Gamma=2.275 \times 10^{5} \frac{\left(\rho Y_{e}\right)^{1 / 3}}{T} \sum_{i}\left(n_{i} / n_{\text {ions }}\right) Z_{i}^{5 / 3}
$$

- Cálculo del proceso bremsstrahlung degenerado: en el caso de altas correlaciones iónicas se utiliza una mezcla de $\mathrm{H}, \mathrm{He},{ }^{12} \mathrm{C},{ }^{13} \mathrm{C}, \mathrm{N},{ }^{16} \mathrm{O},{ }^{17} \mathrm{O},{ }^{18} \mathrm{O},{ }^{20} \mathrm{Ne}$ y ${ }^{22} \mathrm{Ne}$. Se llaman a las rutinas axion_raf_deg cuando $\Gamma<0.9$ y axion_naka cuando $\Gamma>1.1$ para obtener la tasa de pérdida de energía por axiones. Además, se realiza una interpolación de la emisión de axiones para el rango $0.9<\Gamma<1$.1. Luego se calculan las derivadas de $\epsilon_{A}$ de manera aproximada ignorando la dependencia de la constante de acoplamiento Coulombiana con la presión y la temperatura. 
- Cálculo del proceso bremsstrahlung no degenerado: se llama a la rutina axion_ND_brem que determina $\epsilon_{A}$ utilizando la ec. (2.28). Nuevamente se obtienen las derivadas de manera aproximada.

- Por completitud, se incluye la emisión de axiones $\epsilon_{A}$ y sus derivadas por efecto Compton, ver ec. (2.30), a través de la rutina axion_Compton.

- Finalmente, se suman todas las contribuciones indicadas previamente según el régimen que corresponda para obtener la emisión total de axiones del tipo DFSZ en unidades del sistema cegesimal.

\subsection{Cotas existentes}

\subsubsection{AXIONES DE FUENTES ASTROFÍSICAS}

Cotas obTEnIDAs A PARTIR DE PÉRDIDAS DE ENERGía EN ESTRELlaS

En términos generales las partículas de baja masa, débilmente interactuantes (como neutrinos, gravitones, axiones, bosones de gauge bariónicos o leptónicos, etc.), podrían ser producidas en plasmas calientes astrofísicos y, por lo tanto, pueden transportar energía fuera de las estrellas. El acoplamiento de tales partículas con la materia y la radiación se encuentra acotado por la restricción de que los tiempos de vida estelares o las tasas de pérdida de energía sean compatibles con las observaciones, ver Turner (1990); Raffelt (2008).

Se considerará en primer lugar las cotas derivadas del Sol para axiones hadrónicos. Como se dijo antes, este tipo de axiones se produce mediante el proceso Primakoff $\gamma+Z e \rightarrow Z e+A$. Al realizar la integración, considerando el modelo solar estándar, se obtiene para la luminosidad de axiones:

$$
L_{A}=g_{10}^{2} 1.85 \times 10^{-3} L_{\odot}
$$


El máximo del espectro para la luminosidad se encuentra en $3 \mathrm{KeV}$, la media en 4.2 $\mathrm{KeV}$ y, además, el flujo medido en tierra es: $g_{10}^{2} 3.75 \times 10^{11} \mathrm{~cm}^{-2} \mathrm{~s}^{-1}$. Este flujo puede ser acotado a partir del flujo de neutrinos ya que, como la luminosidad de fotones del Sol está fija, las pérdidas por axiones implican una mayor producción de energía nuclear y por tanto un incremento en dicho flujo. La cota más reciente al respecto es $g_{10}<4.1$ (a $3 \sigma$ ), la cual fue obtenida por Vinyoles et al. (2015) por medio de un análisis estadístico combinado de observaciones de heliosismología y de neutrinos solares que incluye tanto errores en la teoría como en las observaciones.

Límites más restrictivos se derivan a partir de estrellas de la rama horizontal (HB) pertenecientes a Cúmulos Globulares (GC). Estas estrellas alcanzan la fase de la quema de He con una energía promedio liberada por el núcleo de $80 \mathrm{erg} \mathrm{g}^{-1} \mathrm{~s}^{-1}$, mientras que las pérdidas por efecto Primakoff son de $g_{10}^{2} 30 \mathrm{ergg}^{-1} \mathrm{~s}^{-1}$. De esta manera, el consumo acelerado de He reduce los tiempos de vida en la HB en un factor de 80/(80+30 $\left.g_{10}^{2}\right)$.

El número de estrellas en la HB relevadas en una muestra de 39 GC galácticos, comparadas con el número de gigantes rojas, las cuales no se encuentran afectadas por el efecto Primakoff significativamente, es un indicador de pérdidas anómalas de energía que pueden ser explicadas por emisión de axiones. La cota superior para la constante de acoplamiento axión-fotón encontrada en este análisis es

$$
\left|g_{a \gamma \gamma}\right|<6.6 \times 10^{-11} \mathrm{GeV}^{-1}
$$

con un $95 \%$ nivel de confianza (CL), ver Ayala et al. (2014). Para axiones KSVZ, la restricción de la ec. (2.35) se traduce a $f_{a}>3.4 \times 10^{7} \mathrm{GeV}\left(m_{a}<0.2 \mathrm{eV}\right)$ para $z=0.56$.

Si los axiones se acoplan directamente a electrones (DFSZ), los procesos de emisión dominante son las recombinaciones y desexcitaciones axiónicas, el bremsstrahlung en las colisiones ion-electrón o electrón-electrón y la dispersión Compton. De esta manera, las estrellas ubicadas en la rama de gigantes rojas del diagrama Color-Magnitud son particularmente sensibles a este tipo de procesos. En este sentido, en Viaux et al. (2013) se desarrolló una fotometría de alta precisión para el cúmulo globular M5 (NGC 5904), permitiendo así una comparación detallada entre el extremo superior observado de la rama de las gigantes y las predicciones basadas en los modelos evolutivos. Los autores 
encontraron que tanto el tip observado como el teórico están en buen acuerdo, lo que da lugar a la siguiente cota

$$
\alpha_{\text {aee }}<1.5 \times 10^{-26}(95 \% \mathrm{CL})
$$

que se traduce a un límite superior para la masa del axión en el modelo DFSZ

$$
m_{a} \cos ^{2} \beta<15 \mathrm{meV}(95 \% \mathrm{CL})
$$

ver figura 2.3. Curiosamente, el acuerdo mejoraría si se considerase una pequeña cantidad de enfriamiento extra durante la quema de He, dando lugar a un valor para la constante de estructura fina axiónica de $\alpha_{\text {aee }} \sim 2.8 \times 10^{-27}$, que corresponde a $m_{a} \cos ^{2} \beta I \sim 7 \mathrm{meV}$. En relación a esto, Giannotti et al. (2016) encontraron, mediante el ajuste simultáneo de las pérdidas de energía extra en las estrellas en la HB y las estrellas en la rama de las gigantes, una constante de acoplamiento axión-fotón $g_{a \gamma \gamma} \sim \times 10^{-12} \mathrm{GeV}^{-1}$ y axión-electrón $\alpha_{a e e} \sim 10^{-27}$.

Como se mencionó anteriormente, el Bremmstrahlung es un proceso eficiente en el interior de las EBs ya que la gran frecuencia del plasma impide que los procesos Primakoff y Compton se lleven a cabo. En este sentido, Raffelt (1986) encontró que, a partir de la comparación de la WDLF observada y teórica, específicamente haciendo uso de los tiempos de enfriamiento, se puede acotar $\alpha_{a e e}$. Asimismo, mediante esta última metodología, se darán a conocer en el capítulo 4 las cotas obtenidas en esta Tesis para axiones del tipo DFSZ. En el caso de las EBs pulsantes (estrellas ZZ Ceti), el decrecimiento en los períodos pulsacionales $\dot{P} / P$ es una medida de la tasa de enfriamiento. Observaciones en esta dirección de las EBs pulsantes G117-B15A y R548 evidencian un enfriamiento adicional, el cual puede explicarse en términos de pérdidas por axiones, ver Córsico et al. (2012a,b). 


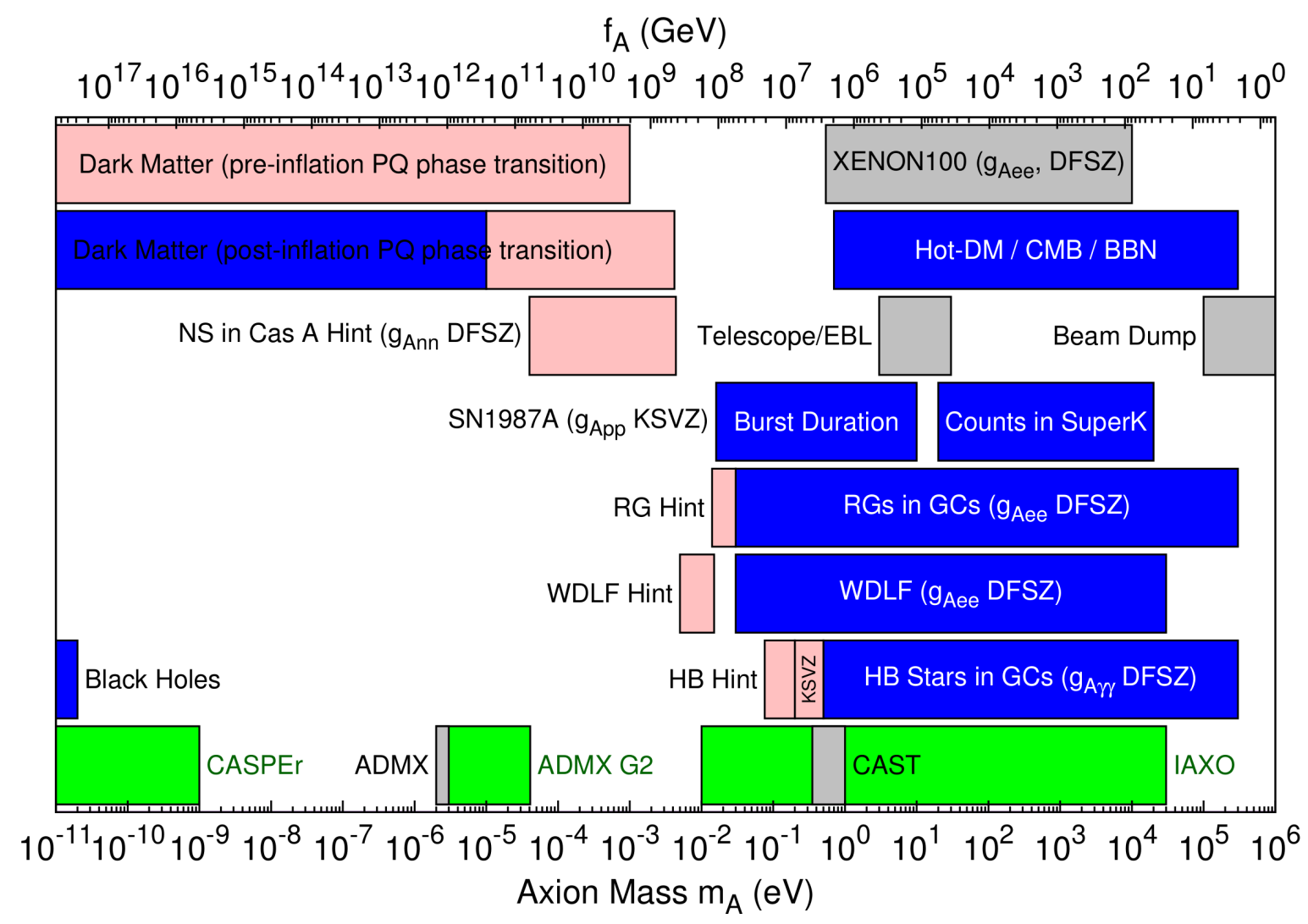

Figura 2.3: Rangos de exclusión como se describen en el texto. Los intervalos en la fila de abajo son los rangos de búsqueda aproximados de los experimentos ADMX, CASPEr, CAST, e IAXO, donde las regiones verdes indican las búsquedas proyectadas. Los límites en las constantes de acoplamientos se trasladan a $m_{a}$ y $f_{a}$ utilizando $z=0.56$ y los valores para el modelo KSVZ, a menos que se mencione lo contrario. Los límites para $g_{a e}$ están determinados por el modelo DFSZ con un acoplamiento axión-electrón correspondiente a $\cos \beta \prime=1 / 2$. Figura extraída de Patrignani et al. (2016).

\section{Cotas obTenidas a PARTIR De BÚsquedas DE AXIONES SOlares Y ALPS}

En lugar de utilizar las pérdidas de energía anómalas en estrellas para obtener cotas para el axión, también es posible buscar directamente el flujo de estas partículas, en especial el proveniente del Sol. Las partículas de tipo axiónicas (ALPs) se producen a través del proceso Primakoff a través de la ec. (2.34) con una energía promedio de $4.2 \mathrm{keV}$ y pueden ser detectadas en tierra por medio de los procesos inversos con ayuda de un campo magnético $\bar{B}$ ("helioscopio axiónico"), ver Sikivie (1983). Con la finalidad de extender la sensibilidad hacia valores más grandes de la masa del axión, es posible 
dotar al fotón de una masa efectiva en el gas, $m_{\gamma}=w_{\text {plasma }}$, equiparándose así las relaciones de dispersión del axión y del fotón, véase el trabajo de van Bibber et al. (1989).

El helioscopio más reciente es el CAST (CERN Axion Solar Telescope), el cual utiliza un imán reciclado del experimento LHC (Large Hadron Collider). Según Andriamonje et al. (2007), este helioscopio ha establecido una cota $\left|g_{a \gamma \gamma}\right|<8.8^{-11} \mathrm{GeV}^{-1}$ para $m_{a}<$ $0.02 \mathrm{eV}$, con un $95 \%$ de CL. Por otra parte, el experimento XENON100 ha presentado sus primeros resultados de la búsqueda de axiones solares y ALPs, ver Aprile et al. (2014). La cota encontrada para la constante de acoplamiento axión-electrón es

$$
g_{a e e}<7.7 \times 10^{-12}(90 \% \mathrm{CL})
$$

excluyendo modelos de axiones DFSZ con $m_{a} \cos ^{2} \beta^{\prime}>0.27 \mathrm{eV}$.

Otras búsquedas del efecto Primakoff para axiones solares y ALPs hacen uso de cristales como detectores, aprovechando la conversión coherente de axiones en fotones cuando el ángulo de incidencia del axión satisface una condición de Bragg con un plano del cristal. Sin embargo, los límites derivados son menos restrictivos que los obtenidos de la cota a la luminosidad de axiones $L_{A}$ mencionada más arriba.

Por último, otro método para la detección de este tipo de partículas es la utilización de satélites de rayos $X$ que apunten al Sol, justo cuando la Tierra se encuentra en el medio de ellos. De esta manera, los axiones solares y ALPs se convertirían en presencia del campo magnético terrestre y podrían ser detectados. La sensibilidad a $g_{a \gamma \gamma}$ podría ser comparable a la de CAST, aunque solo para masas mucho más pequeñas. En Hudson et al. (2012), se han reportado límites preliminares para $g_{a \gamma \gamma}$ a partir de medidas de rayos $X$ solares efectuadas con satélites existentes.

\section{Cotas obtenidas a PARTIR De Flujos astrofísicos}

En el contexto astrofísico, existen campos magnéticos de gran escala que pueden inducir oscilaciones axión-fotón. Un ejemplo es la SN 1987A, la cual podría emitir un burst de ALPs mediante el proceso Primakoff en su núcleo, con éstas partículas convirtiéndose 
parcialmente luego en rayos $\gamma$ en el campo magnético galáctico. La falta de señal de rayos $\gamma$ en el espectrógrafo (GRS) del satélite "Solar Maximum Mission" (SMM) en consonancia con la observación de los neutrinos emitidos por SN 1987A brindan entonces cotas muy restrictivas con el acoplamiento con fotones. Este tipo de estudio ha dado lugar recientemente a la siguiente cota para la constante de acoplamiento axión-fotón en la región $m_{a}<4.4 \times^{-10} \mathrm{eV}$ :

$$
\left|g_{a \gamma \gamma}\right|<5.3 \times 10^{-12} \mathrm{GeV}^{-1},
$$

ver Payez et al. (2015). Las oscilaciones magnéticas inducidas entre fotones y ALPs pueden modificar el flujo de fotones de fuentes distantes de distintas maneras: atenuándolo de manera dependiente de la frecuencia, variando la polarización, y evitando la absorción por propagación en forma de axiones. Las búsquedas de emisión linealmente polarizada en EBs magnetizadas y cambios de polarización en radio galaxias proveen límites cercanos a $g_{a \gamma \gamma} \sim 10^{-11} \mathrm{GeV}^{-1}$ para masas $m_{a}<10^{-7} \mathrm{eV}$, ver Gill \& Heyl (2011) y Horns et al. (2012). Cotas aún más restrictivas se han obtenido en el trabajo de Payez et al. (2012) a partir de medidas de alta precisión de polarización en quasares:

$$
g_{a \gamma \gamma}<2 \times 10^{-13} \mathrm{GeV}^{-1} \text { para } \mathrm{m}_{\mathrm{a}}<10^{-14} \mathrm{eV} .
$$

Por otra parte, la región de parámetros $g_{a \gamma \gamma} \sim 10^{-12}-10^{-10} \mathrm{GeV}^{-1}$ para $m_{a}<$ $10^{-7} \mathrm{eV}$, la cual es interesante para determinar el rol de las oscilaciones fotón-ALP en la excesiva transparencia del Universo a rayos $\gamma$ del orden del $\mathrm{TeV}$, puede ser testeada mediante la búsqueda de un comportamiento irregular del espectro de rayos $\gamma$ de núcleos de galaxias activos (AGNs). En relación a esto, la colaboración "The High Energy Stereoscopic System" (H.E.S.S), ver Abramowski et al. (2013), ha establecido un límite de

$$
\left|g_{a \gamma \gamma}\right|<2.1 \times 10^{-11} \mathrm{GeV}^{-1} \text { para } 1.5 \times 10^{-8} \mathrm{eV}<\mathrm{m}_{\mathrm{a}}<6.0 \times 10^{-8} \mathrm{eV} \text {, }
$$

a partir de la observación del espectro del AGN PKS 2155, ver figura 2.4. 


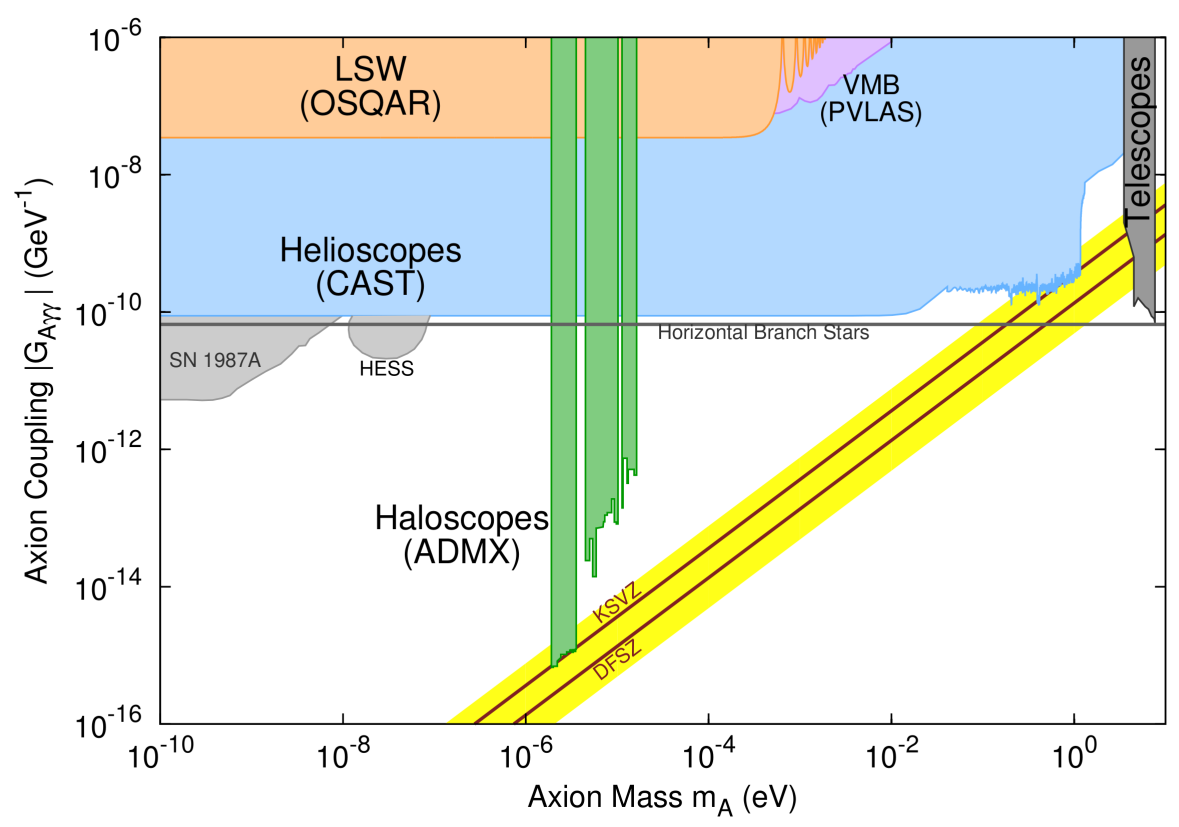

Figura 2.4: Figura que muestra los rangos de exclusión para las ALPs, como se describen en el texto, ver Patrignani et al. (2016).

\section{SUPERRADIANCIA DE AGUJEROS NEGROS}

Campos bosónicos ultralivianos tales como los axiones o, más generalmente, ALPs pueden afectar la dinámica y la emisión de ondas gravitacionales en agujeros negros que rotan rápidamente a través del mecanismo de superradiancia de Penrose. La existencia de campos bosónicos ultralivianos desestabilizantes se manifiesta en la presencia de gaps en la gráfica de masa vs. espín de agujeros negros rápidamente rotantes. Así, las medidas de espín en agujeros negros excluyen un rango de masas $6 \times 10^{-13} \mathrm{eV}<\mathrm{m}_{\mathrm{a}}<2 \times$ $10^{-11} \mathrm{eV}$ a $2 \sigma$, ver Arvanitaki et al. (2010); Arvanitaki \& Dubovsky (2011). Asimismo, en el contexto del mecanismo de superradiancia, los axiones pueden producir ondas gravitacionales monocromáticas de largo alcance las cuales pueden ser distinguidas de fuentes astrofísicas ordinarias. Por lo tanto, el detector de ondas gravitacionales LIGO (de las siglas en inglés Laser Interferometer Gravitational-Wave Observatory) debería ser sensible a masas para el axión del orden de $\sim 10 \times^{-10} \mathrm{eV}$. 


\subsubsection{AXIONES CÓSMICOS}

El principal interés en el axión desde el punto de vista cosmológico reside en su posible rol como candidato a materia oscura fría (CDM). En el régimen de masas correspondiente, distintas búsquedas han sido desarrolladas a través de telescopios, cavidades de microondas y técnicas de resonancia magnética.

En lo que respecta a las cotas obtenidas a partir del uso telescopios, Blout et al. (2001) realizaron una búsqueda con el radio telescopio Haystack en tres galaxias enanas cercanas teniendo como señal una línea de emisión cuasimonocromática. Como resultado de dicha búsqueda se ha reportado un límite de

$$
g_{a \gamma \gamma}<1.0 \times 10^{-9} \mathrm{GeV}^{-1} \text { para } 298 \mu \mathrm{eV}<\mathrm{m}_{\mathrm{a}}<363 \mu \mathrm{eV} .
$$

Es preciso notar que esta combinación para $m_{a}$ y $g_{a \gamma \gamma}$ no excluye modelos plausibles de axiones.

En el rango de masas plausible según CDM, axiones provenientes del halo galáctico podrían ser detectados mediante su conversión resonante a una señal de microondas cuasimonocromática en una cavidad electromagnética sumergida en un fuerte campo estático $\bar{B}$. El "Axion Dark Matter Experiment" (ADMX) ha conseguido ser sensible a axiones KSVZ en el rango de masas $1.9-3.3 \mu \mathrm{eV}$ suponiendo que estos saturan la densidad local de materia oscura y están suficientemente virializados, ver Asztalos et al. (2004).

Finalmente, las búsquedas de resonancia magnética se basan en el hecho de que el campo oscilante de axiones asociado a la materia oscura galáctica induce momentos dipolares eléctricos (EDMs) nucleares. Estos EDMs provocan la precesión de los espines nucleares en una muestra polarizada de nucleones en presencia de un campo eléctrico. En el caso del "Cosmic Axion Spin Precession Experiment" (CASPEr), ver Budker et al. (2014), se busca probar las regiones $f_{a}>10^{15} \mathrm{GeV}$, correspondiente a escalas para la masa del axión menor a los nanovoltios, que son complementarias a aquellas exploradas con las técnicas de cavidad. 


\subsubsection{Búsquedas DE LABORATORIO}

En general las búsquedas de laboratorio de axiones invisibles se basan en tratar la conversión axión-fotón en un campo macroscópico, usualmente un campo magnético extendido una distancia $L$ grande, como un fenómeno de oscilación análogo a las oscilaciones de neutrinos. Algunos experimentos consideran fotones propagándose a través de dos campos magnéticos transversos separados por una barrera óptica y analizan la regeneración de fotones a partir del haz puro de axiones que atraviesa dicha barrera. Otros experimentos, en cambio, estudian propiedades de polarización propias del haz, más precisamente, efectos de dicroísmo y birrefringencia inducidos por la conversión fotón-axión. En el primer caso, la cota más restrictiva fue obtenida por el experimento OSQAR (Optical Search for QED Vacuum Birefringence, Axions, and Photon Regeneration), ver Ballou et al. (2015):

$$
\left|g_{a \gamma \gamma}\right|<3.5 \times 10^{-8} \mathrm{GeV}^{-1} \text { para } \mathrm{m}_{\mathrm{a}}<0.3 \mu \mathrm{meV}
$$

con un $95 \%$ de CL. Además, en el contexto de experimentos que buscan incrementar resonantemente la regeneración de los fotones, cabe mencionar que, para $m_{a}<10^{-5} \mathrm{eV}$, la cota establecida por el experimento CROWS (CERN Resonant Weakly Interacting sub-eV Particle Search) es levemente menos restrictiva que aquella obtenida por OSQAR, ver Betz et al. (2013). Con respecto a la búsqueda de efectos de birrefringencia y dicroísmo, en Semertzidis et al. (1990) se ha establecido una cota para la constante de acoplamiento $\left|g_{a \gamma \gamma}\right|<3.6 \times 10^{-7} \mathrm{GeV}^{-1}$ con el $95 \%$ de CL para $m_{a}<5 \times 10^{-4} \mathrm{eV}$.

Una búsqueda de éstos efectos fue realizada en un dipolo magnético, ver Semertzidis et al. (1990), donde la rotación debida a efectos de dicroísmo otorga una fuerte cota para la constante de acoplamiento $\left|g_{a \gamma \gamma}\right|<3.6 \times 10^{-7} \mathrm{GeV}^{-1}$ con el $95 \%$ de CL para $m_{a}<5 \times 10^{-4} \mathrm{eV}$. 



\section{3}

\section{Cotas para la constante de acoplamiento axión-fotón a partir de estrellas Cefeidas}

En este capítulo se estudia el impacto de la emisión de axiones KSVZ (descriptos en el capítulo 2) por efecto Primakoff en el interior de estrellas Cefeidas Clásicas. A partir del análisis del comportamiento de los tiempos durante la quema de He con respecto a la constante de acoplamiento axión-fotón $\left(g_{a \gamma \gamma}\right)$, la cual regula la contribución anómala a la pérdida de energía, se examinarán las conclusiones obtenidas por Friedland et al. (2013). Más aún, a través de la inspección de las secuencias evolutivas, se recupera el criterio propuesto en dicho trabajo para acotar $g_{a \gamma \gamma}$, el cual fue anticipado en la sección 1.1 y establece que aquellos valores para los cuales la fase de blue loop es completamente eliminada deben ser descartados por resultar incompatibles con las observaciones. Ahora bien, independientemente de las pérdidas anómalas de energía, la etapa evolutiva de blue loop puede verse afectada por los cambios en los parámetros físicos y/o por las hipótesis físicas empleadas en los cálculos evolutivos como se detallará a continuación. En contraposición a las estrellas de baja masa, en el caso de las estrellas de masa intermedia el comportamiento del blue loop ante dichos cambios y suposiciones físicas no puede ser predicho fácilmente. Esto se debe a que en las estrellas 
de masa intermedia, la contribución a la energía de la estrella proporcionada por la quema en capa del $\mathrm{H}$ es significativamente mayor que en las estrellas de baja masa. A su vez, las contribuciones relativas de energía correspondientes a la quema de He e H en las capas cambian apreciablemente la quema de He en el núcleo. En consecuencia, cualquier variación de los parámetros físicos de entrada que modifique la eficiencia de la quema de $\mathrm{H}$, puede potenciar o inhibir la fase de blue loop. La dependencia del blue loop con los parámetros físicos más importantes puede resumirse como sigue:

- Masa de la estrella: para estrellas con masas por debajo de 10-12M $\odot$, la extensión del blue loop generalmente se incrementa con la masa. En estrellas más masivas, el blue loop desaparece ya que la estrella es capaz de encender el He antes de llegar a la línea de Hayashi.

- Composición química: en general puede decirse que si se incrementa la abundancia inicial de He, la extensión del blue loop es más grande. Lo contrario ocurre cuando se incrementa la abundancia de elementos pesados. Sin embargo, este comportamiento se contradice con algunos cálculos evolutivos que muestran que el comportamiento del blue loop con el contenido de He y de elementos pesados es no lineal, ver Renzini et al. (1992) y Salaris \& Cassisi (2005).

- Procesos de mezcla: al incrementar la eficiencia del overshooting nuclear durante toda la evolución, la extensión del blue loop se reduce. Por otra parte, la extensión del blue loop aumenta cuando el overshooting en la envoltura es incrementado*. Este comportamiento está de acuerdo con lo afirmado en Halabi et al. (2012). Sin embargo, el efecto parece disminuir a medida que se incrementa la masa de la estrella. A diferencia de lo que ocurre con la composición química, en este caso la forma del blue loop se ve apenas modificada.

Dado que la cota establecida en Friedland et al. (2013) ignora el mencionado impacto de los parámetros físicos sobre el blue loop, se extiende en este capítulo el análisis realizado por Friedland et al. (2013) a fin de determinar hasta qué punto la cota para $g_{10}$ se degrada al variar tales parámetros. En particular, por medio del cómputo de 160

\footnotetext{
${ }^{*}$ Los parámetros de overshooting serán apropiadamente introducidos en la sección 3.1
} 
simulaciones numéricas que involucran estrellas en el rango de masas $6 \mathrm{M}_{\odot}-10 \mathrm{M}_{\odot}$, se muestra que la sensibilidad a $g_{a \gamma \gamma}$ presenta una dependencia con la masa estelar y que, efectivamente, la cota para dicho acoplamiento se relaja al considerar las incertezas provenientes del modelado de los bordes convectivos durante la quema central del He. Finalmente, a la luz de las cotas obtenidas en cada uno de los escenarios supuestos para los parámetros físicos, se establece una cota global robusta para $g_{10}=g_{a \gamma \gamma} 10^{10} \mathrm{GeV}$.

\subsection{Herramientas Numéricas: Física COnstitutiva y SECUEnCias evoluti- VAS}

Las secuencias evolutivas calculadas en este capítulo fueron realizadas con una versión actualizada del código de evolución estelar LPCODE descripto en el apéndice A. Se construyeron modelos numéricos de estrellas Cefeidas mediante el cálculo de la evolución completa de modelos de estrellas de masa intermedia desde la secuencia principal hasta el final de la quema de He.

La física constitutiva utilizada en la simulación de las secuencias evolutivas de las Cefeidas puede especificarse como sigue. Durante la quema central de $\mathrm{H}$ y $\mathrm{He}$ en el núcleo, la ecuación de estado utilizada incorpora a altas densidades los efectos de presión de radiación, gas ideal, gas parcialmente degenerado y correcciones coulombianas. Por otra parte, en el caso de bajas densidades, se tienen en cuenta gas no degenerado, ionización parcial del $\mathrm{H} / \mathrm{He}$ y la presión de radiación. La ionización parcial es tratada mediante ecuaciones de Saha (sin interacciones) y esta provee las cantidades termodinámicas requeridas por el código evolutivo. Con respecto a las opacidades radiativas, se ha utilizado el proyecto OPAL, Iglesias \& Rogers (1996), las cuales se suplementan, a bajas temperaturas, con las opacidades moleculares de Alexander \& Ferguson (1994). Respecto a las tasas de las reacciones nucleares involucradas, se han adoptado los dados en Kunz et al. (2002) y Fynbo et al. (2005) para las reacciones ${ }^{12} C(\alpha, \gamma){ }^{16} O$ y $3 \alpha$, respectivamente, mientras que para las demás reacciones se han usado los valores de NACRE, ver Angulo et al. (1999), y Caughlan \& Fowler (1988).

La mezcla convectiva para estrellas de masa intermedia es tratada en el LPCODE resolviendo la siguiente ecuación de difusión dependiente del tiempo 


$$
\frac{d X_{i}}{d t}=\left(\frac{\partial X_{i}}{\partial t}\right)_{n u c}+\frac{\partial}{\partial M_{r}}\left[(4 \pi \rho)^{2} D \frac{\partial X_{i}}{\partial M_{r}}\right]
$$

donde $X_{i}$ son las componentes de un vector que contiene las abundancias de todos los elementos. En la ec. (3.1), el primer término describe la evolución nuclear y está completamente acoplado al cambio en la composición real debido a los procesos de mezcla, el cual está representado por el segundo término. En particular, la mezcla convectiva está descripta por el coeficiente de difusión $D$, cuya forma funcional depende del modelo de mezcla elegido en el código numérico. Para las regiones inmediatamente adyacentes a las zonas convectivamente inestables se ha adoptado un coeficiente de difusión dependiente de la profundidad siguiendo la prescripción de Freytag et al. (1996):

$$
D(z)=D_{o} e^{\frac{-2 z}{f H_{p}}}
$$

donde $z$ denota la distancia a la zona del borde convectivo, $H_{p}$ es la altura de escala de la presión y $D_{o}$ es el coeficiente de difusión cerca del borde de la región convectiva. A su vez, el parámetro $f$ (parámetro de overshooting) es una medida de la eficiencia de la mezcla parcial extra y define las velocidades de los elementos convectivos más allá del límite de las zonas inestables. Asimismo, el coeficiente de difusión se considera distinto de cero mientras $D / D_{o}>10^{-10}$. Por debajo de ese valor, no se considera mezcla.

Para el cálculo de las secuencias evolutivas de Cefeidas, se ha incluido mezcla por overshooting tanto en el núcleo como en la envoltura de los modelos estelares, usándose, en particular, los siguientes valores: $f_{n u c}=0.0174,0.01$ y $f_{\text {env }}=0.0174,0.01$. El valor $f=0.0174$ se establece a partir de la calibración de la secuencia principal superior y es equivalente a extender el overshooting en 0.2 veces la altura de escala de presión. Por otra parte, $f=0.01$ es el valor empleado en las simulaciones numéricas de estrellas de masa intermedia desarrolladas por Friedland et al. (2013). Sin embargo, debido a que el cut-off utilizado por el código de evolución estelar MESA, ver Paxton et al. (2013), es distinto al del LPCODE, el valor $f=0.01$ no resulta estrictamente comparable con el utilizado por Friedland et al. (2013).

En cuanto a las secuencias evolutivas de las Cefeidas, las mismas fueron obtenidas a partir del cálculo de la evolución completa desde la ZAMS hasta la finalización de 
la quema de He para estrellas de $6 \mathrm{M}_{\odot}, 7 \mathrm{M}_{\odot}, 8 \mathrm{M}_{\odot}, 9 \mathrm{M}_{\odot}$ y $10 \mathrm{M}_{\odot}$, considerando una composición inicial $(X, Y, Z)=(0.708,0.272,0.02)$, similar a la composición utilizada por Friedland et al. (2013).

\subsection{COMPARACIÓN CON EL CÓDIGO MESA}

Antes de realizar el estudio del impacto de la emisión de axiones sobre la duración de la quema central del He y la fase de blue loops, es preciso analizar las posibles diferencias sistemáticas existentes en las escalas de tiempo evolutivas obtenidas mediante el código LPCODE y el código MESA utilizado por Friedland et al. (2013). Por tanto, se han comparado en esta Tesis las simulaciones del LPCODE con aquellas correspondientes al MESA, ver Paxton et al. (2013), para estrellas con $M=3-8 M_{\odot}$ sin emisión de axiones KSVZ. Más concretamente, se han contrastado los tiempos en la quema central de H y He $\left(\Delta t_{H}\right.$ y $\Delta t_{H e}$, respectivamente) con aquellos presentados en Paxton et al. (2013), los cuales fueron calculados sin la incorporación de overshooting ni de ninguna mezcla extra. En ambos casos los modelos evolucionaron desde la ZAMS hasta el final de la quema de He en el núcleo y tienen una composición química inicial correspondiente a $Y=0.272$ y $Z=0.02$. Cabe mencionar, además, que no se incluyeron la pérdida de masa ni la rotación. Finalmente, los bordes de las zonas de mezcla están determinados por el criterio de Schwarzschild con $\alpha_{M L T}=1.88$.

La tabla 3.1 muestra los resultados correspondientes a los tiempos evolutivos en la quema central de $\mathrm{H}$ y He para todas las secuencias evolutivas calculadas. Los tiempos evolutivos se definen como el intervalo entre el comienzo de la quema central de $\mathrm{H}(\mathrm{He})$ y su agotamiento hasta el $1 \%$ de la abundancia central en el núcleo. De dicha tabla, puede notarse que las diferencias en $\Delta t_{H}$ son menores al $4 \%$, mientras que en el caso de $\Delta t_{H e}$ resultan ser menores al $12 \%$. Este último porcentaje es entonces la incerteza a tener en cuenta para comparar consistentemente los resultados obtenidos en esta Tesis al incluir pérdidas anómalas por axiones con aquellos reportados en Friedland et al. (2013). 


\begin{tabular}{ccccccc}
\hline \hline \multirow{2}{*}{$M / M_{\odot}$} & \multicolumn{2}{c}{$L P C O D E$} & & \multicolumn{2}{c}{ MESA } \\
\cline { 2 - 3 } \cline { 6 - 7 } & \multicolumn{2}{c}{$\Delta t_{H}$} & $\Delta t_{H e}$ & & $\Delta t_{H}$ & $\Delta t_{H e}$ \\
\hline 3.0 & 308.78 & 94.05 & & 320.6 & 83.59 \\
4.0 & 147.18 & 32.69 & & 152.7 & 29.78 \\
5.0 & 84.96 & 16.63 & & 85.61 & 15.52 \\
6.0 & 55.67 & 10.32 & & 55.98 & 9.62 \\
7.0 & 39.84 & 6.97 & & 39.91 & 6.51 \\
8.0 & 30.36 & 4.9 & & 30.42 & 4.67 \\
\hline \hline
\end{tabular}

Tabla 3.1: Tiempos evolutivos en Myr durante la quema central de $\mathrm{H}$ y He obtenidos con los códigos de evolución estelar LPCODE y MESA para estrellas con $M=3-8 M_{\odot}$

\subsection{IMPACTO DE LA EMISIÓN DE AXIONES DURANTE LA EVOLUCIÓN}

Según lo analizado en el trabajo de Friedland et al. (2013) los axiones aceleran la evolución durante la quema de He, de esta manera su impacto queda de manifiesto al graficar la temperatura efectiva $\left(T_{e f f}\right)$ en términos de la edad en Myr para una estrella representativa de $9.5 M_{\odot}$, ver Carosi et al. (2013). En la figura 3.1 se muestra dicha gráfica considerando distintas contribuciones de axiones dadas por $g_{10}=0.0,0.6,1.2,2$ y 3. Asimismo, se han adoptado los mismos parámetros de overshooting utilizados en Friedland et al. (2013) y en Carosi et al. (2013): $f_{n u c}=0.01$ y $f_{\text {env }}=0.01$ durante la quema de $\mathrm{H}$ en el núcleo, y $f_{n u c}=0$ y $f_{\text {env }}=0.01$ durante la quema de He. La curva roja corresponde al caso estándar, es decir, $g_{10}=0$. Allí se observan tres estadios bien definidos: la quema de $\mathrm{H}$, que dura aproximadamente $24.5 \mathrm{Myr}$, la fase de super gigante roja que se extiende entre 24.5 Myr y 25.8 Myr y, finalmente, la fase de blue loop cuya duración se extiende de 25.8 Myr a 27.3 Myr. Una vez que el He es agotado en el núcleo, éste se contrae hasta encender el Carbono, luego de lo cual las pérdidas por neutrinos se incrementan tanto que el resto de la evolución se produce en un tiempo muy corto. La inclusión de las pérdidas de energía anómalas por axiones acelera la etapa de la quema 


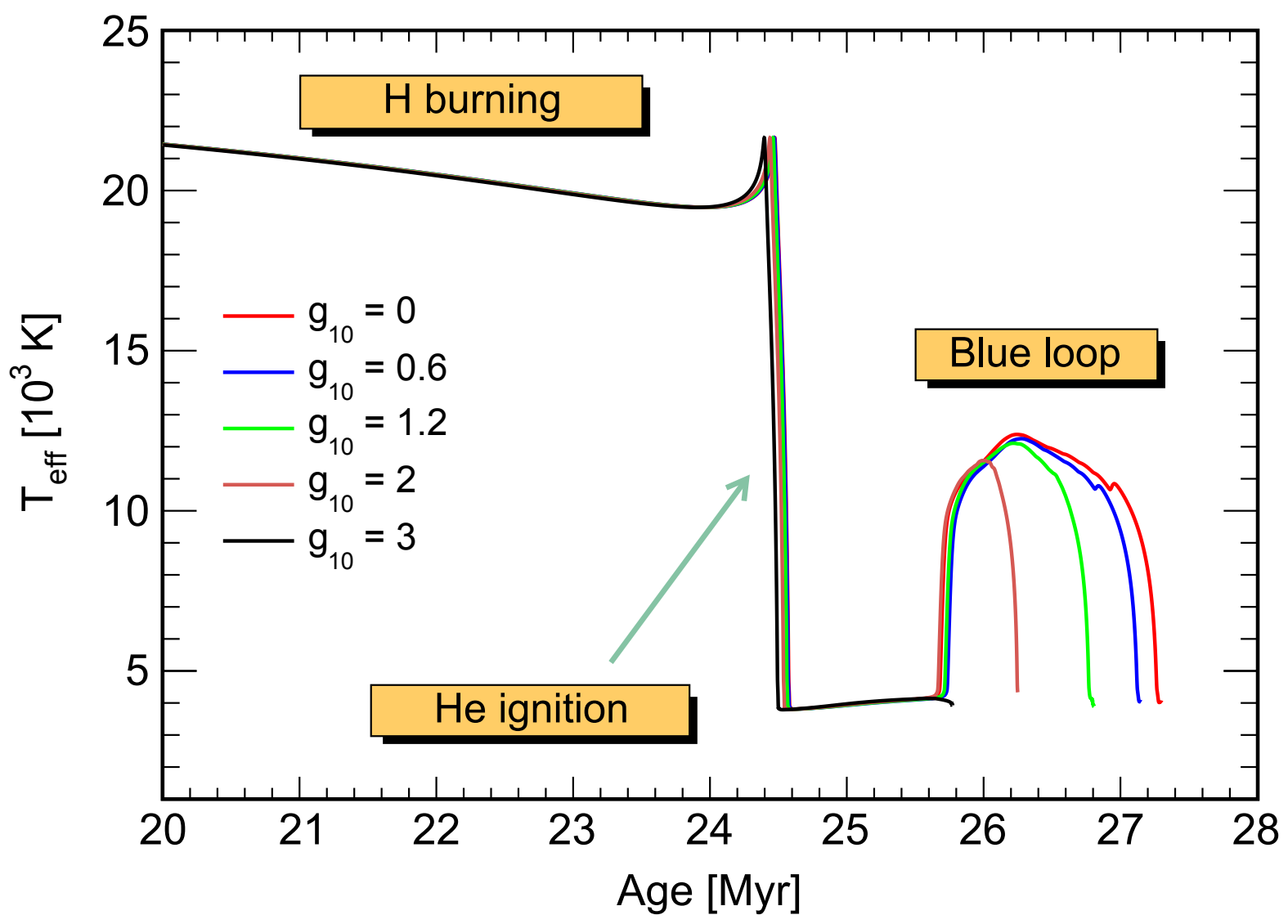

Figura 3.1: Consecuencias del enfriamiento anómalo por axiones en la evolución de una estrella de $9.5 \mathrm{M}_{\odot}$ al usar los mismos parámetros físicos que en el trabajo de Carosi et al. (2013). Se muestran los perfiles de temperatura efectiva correspondientes a $g_{10}=0.0,0.6,1.2,2$ y 3 .

de He, pero no de manera uniforme. En efecto, mientras que la etapa de gigante roja mantiene casi la misma duración, la fase de blue loop es acelerada significativamente a medida que el acoplamiento $g_{10}$ aumenta. Más aún, para acoplamientos tan grandes como $g_{10}=3$ el blue loop es completamente suprimido, como puede apreciarse en la figura 3.2. Este resultado es consistente con el hecho de que para estrellas con masas $\lesssim 10 M_{\odot}$ el comienzo del blue loop es controlado por la quema en capa de H (Friedland et al., 2013; Renzini et al., 1992), mientras que el final de dicha fase es gobernada por la quema de He en el núcleo. Ahora bien, las pérdidas por axiones impactan sobre el núcleo, haciendo que la quema de He se acelere, pero no sobre la capa de H donde la temperatura es más baja. De esta manera, la quema acelerada de He da lugar a un cambio cualitativo en la evolución, es decir, la eliminación del blue loop, ver figuras 3.1 y 3.2. Dado que las Cefeidas se encuentran dentro de la banda de inestabilidad, la desaparición del 


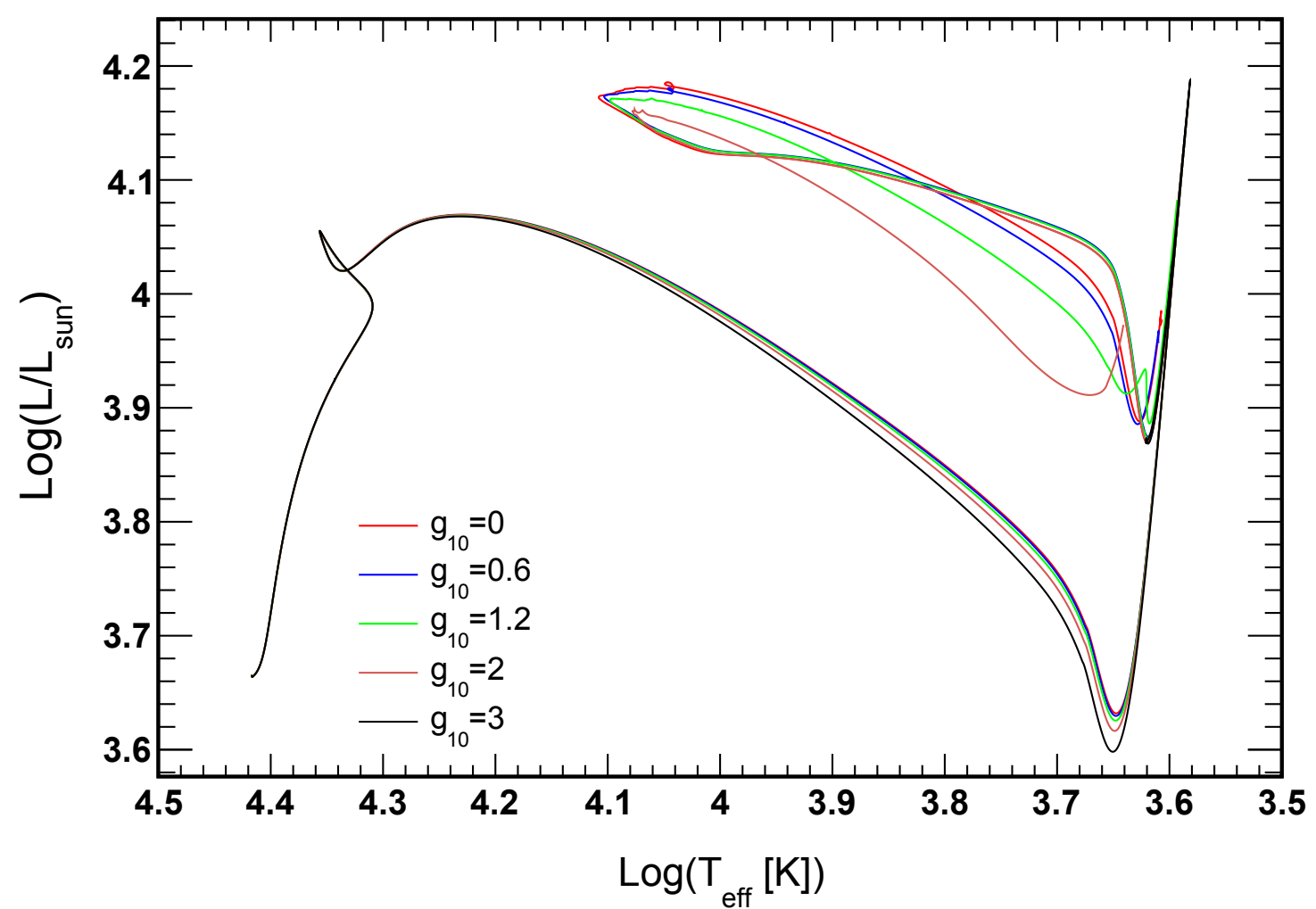

Figura 3.2: Secuencias evolutivas para una estrella representativa de $9.5 M_{\odot}$ con los mismos parámetros de overshooting que los utilizados por Friedland et al. (2013) y Carosi et al. (2013) con $g_{10}=0,0.6,1.2,2$ y 3 .

blue loop no permitiría dar cuenta de la existencia de tales estrellas, ya que el cruce inicial de dicha banda (Gap de Hertzsprung) sucede demasiado rápido como para dar un número suficientemente grande de estas variables. Por lo tanto, aquellos valores de $g_{10}$ que provoquen la eliminación completa de la fase de blue loop resultan incompatibles con las observaciones por lo que deben ser descartados. Así, es posible determinar una cota superior para $g_{10}$ requiriendo la desaparición de la fase de blue loop, es decir, la eliminación de la población completa de estrellas Cefeidas. Este es el argumento propuesto por Friedland et al. (2013) para acotar la constante de acoplamiento axiónfotón.

La figura 3.3 muestra el efecto de la emisión anómala sobre los tiempos evolutivos para estrellas con $6 M_{\odot} \leq M \leq 10 M_{\odot}$ teniendo en cuenta los siguientes parámetros de overshooting: $f_{n u c}=0.0174$ y $f_{\text {env }}=0.0174$ durante la quema de $\mathrm{H}$ en el núcleo, $f_{\text {nuc }}=0.0174$ y $f_{\text {env }}=0.0174$ durante la quema de He. 

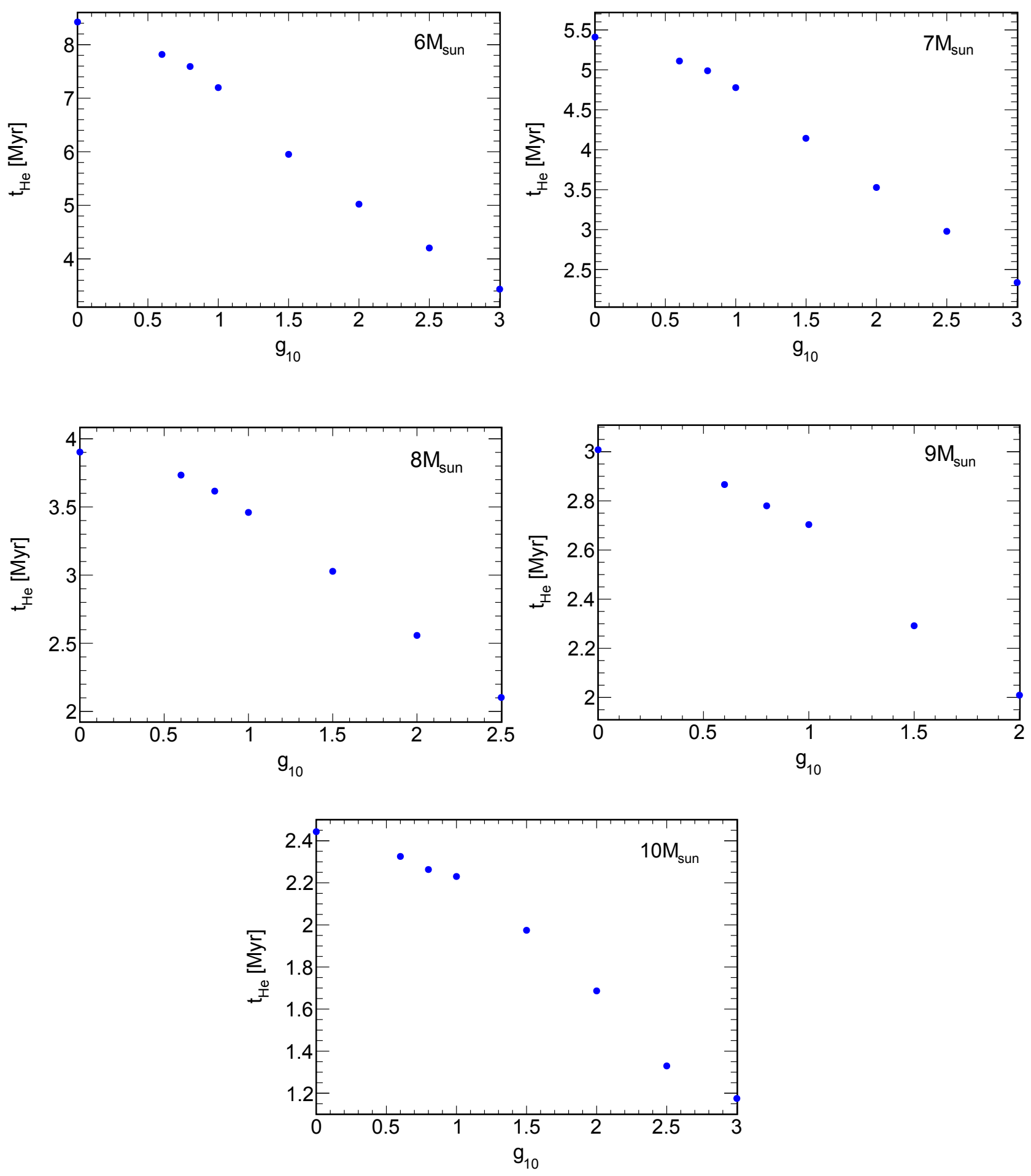

Figura 3.3: Duración de la quema central de He en Myr para estrellas en el rango de $6 M_{\odot} \leq M \leq 10 M_{\odot}$ en función de la constante de acoplamiento axión-fotón $g_{10}$ teniendo en cuenta los siguientes parámetros de overshooting: $f_{n u c}=0.0174$ y $f_{e n v}=0.0174$ durante la quema de $\mathrm{H}$ en el núcleo, $f_{n u c}=0.0174$ y $f_{\text {env }}=0.0174$ durante la quema de He. 
La elección de estos parámetros se fundamenta en el hecho de que son los que ajustan con mayor exactitud a las observaciones, ver Fernie et al. (1995). Para todas las masas consideradas, se observa que el tiempo transcurrido durante la quema de He decrece monótonamente con la constante de acoplamiento $g_{10}$. Más precisamente, si se compara el caso estándar $\left(g_{10}=0.0\right)$ con la emisión anómala para $g_{10}=2$, se obtiene una disminución en el tiempo durante la quema de He del $40 \%$ como máximo. El factor con el que el tiempo durante la quema de He se reduce respecto del caso estándar depende de la masa de la estrella. En efecto, mediante el ajuste por mínimos cuadrados de las gráficas de la figura 3.3 utilizando la forma funcional $t_{\mathrm{He}}=t_{\mathrm{He}}\left(g_{10}=0\right) /\left(1+A g_{10}^{2}\right)$, se obtienen para el parámetro de ajuste $A$ los valores 0.165 y 0.121 para masas de $6 M_{\odot}$ y $10 M_{\odot}$, respectivamente, es decir, existe una variación de casi el $27 \%$ en dicho parámetro en el rango de masas considerado. Por otro lado, de las secuencias evolutivas asociadas a la figura 3.3, se obtuvo que la extensión de la fase evolutiva blue loop también disminuye al aumentar la constante de acoplamiento, por lo que es esperable que, eventualmente, para algún valor de $g_{10}$, dicha fase desaparezca completamente. En consecuencia, las conclusiones derivadas más arriba para la secuencia evolutiva correspondiente a $9.5 M_{\odot}$ acerca del impacto de los axiones en la duración de la fase de blue loop y, especialmente, el argumento de Friedland et al. (2013), se extienden al rango de masas $6 M_{\odot} \leq M \leq$ $10 M_{\odot} \cdot$

\subsection{Cotas derivadas a PARTiR De la Desaparición Del Blue Loop}

A fin de encontrar una cota robusta para la constante de acoplamiento axión-fotón $g_{10}$ por medio del argumento de Friedland et al. (2013), es necesario estudiar el impacto que sobre la misma tienen las incertezas asociadas a la estructura y evolución estelar. Como se desprende de lo dicho en la introducción al presente capítulo, es fundamental tener en cuenta distintas masas y distintos parámetros para el overshooting tanto nuclear como en la envoltura. En efecto, tanto los cambios en la masa como en los procesos de mezcla tienen un impacto directo en la extensión del blue loop y no así en su forma, es decir, producen el mismo efecto cualitativo que la inclusión de axiones durante la evolución estelar. Teniendo esto en cuenta, se han computado modelos con masas en el rango de 
$6 M_{\odot} \leq M \leq 10 M_{\odot}$ y se han definido distintos conjuntos de parámetros físicos a partir de la especificación de los valores correspondientes al overshooting nuclear $\left(f_{\text {nuc }}\right)$ y en la envoltura $\left(f_{\text {env }}\right)$, ver tabla 3.2. Vale recordar que la composición química es la correspondiente a Cefeidas Clásicas del disco Galáctico: $((X, Y, Z)=(0.708,0.272,0.02))$. El cálculo de la cota para $g_{10}$ en cada uno de los escenarios de la tabla 3.2 es entonces lo que permite analizar en qué grado dicha cota se degrada cuando parámetros determinantes de la microfísica son variados.

\begin{tabular}{lcc}
\hline \hline & $f_{\text {nuc }}$ & $f_{\text {env }}$ \\
\hline SET1 & 0.0174 & 0 \\
SET2 & 0.01 & 0 \\
SET3 & 0 & 0.0174 \\
SET4 & 0 & 0.01 \\
SET5 & 0.0174 & 0.0174 \\
\hline \hline
\end{tabular}

Tabla 3.2: Definición de los SETs de parámetros en términos del overshooting nuclear y en la envoltura.

A partir de una grilla de 160 simulaciones numéricas, correspondientes a masas estelares entre $6 M_{\odot}$ y $10 M_{\odot}$ y constantes de acoplamiento axión-fotón entre 0 y 3 , se derivaron en primer lugar cotas para cada uno de los SETs definidos en la tabla 3.2. Las cotas mencionadas fueron determinadas, para cada SET y cada masa, como el valor de $g_{10}$ para el cual el loop desaparece en la correspondiente secuencia evolutiva. Los resultados obtenidos en cada caso se listan en la tabla 3.3. A modo de ilustrar cómo fueron obtenidas cada una de las entradas de la tabla en cuestión, se incluyen en la figura 3.4 las secuencias evolutivas correspondientes al SET1 de parámetros para una estrella de $7 M_{\odot}$ con $g_{10}=0,0.6,0.8,1,1.5,2$ y 2 . Como se observa en la figura, para $g_{10}=1$ la extensión del loop comienza a disminuir considerablemente, desapareciendo completamente para $g_{10}=2.5$, lo que da entonces lugar a la entrada en la segunda fila y la primera columna de la tabla 3.3. 


\begin{tabular}{cccccc}
\hline \hline & $S E T 1$ & $S E T 2$ & $S E T 3$ & SET4 & SET5 \\
\hline $6 M_{\odot}$ & 3 & 3 & 2 & 0.6 & 3 \\
$7 M_{\odot}$ & 2.5 & 2.5 & 1.5 & 0.6 & 3 \\
$8 M_{\odot}$ & 2 & 2 & 2 & 1.5 & 2.5 \\
$9 M_{\odot}$ & 1.5 & 2 & 2 & 2 & 2 \\
$10 M_{\odot}$ & 3 & 3 & 2.5 & 2 & 3 \\
\hline \hline
\end{tabular}

Tabla 3.3: Valor de la cota para $g_{10}$ obtenido para cada masa estelar con cada uno de los conjuntos de parámetros definidos en la tabla 3.2.

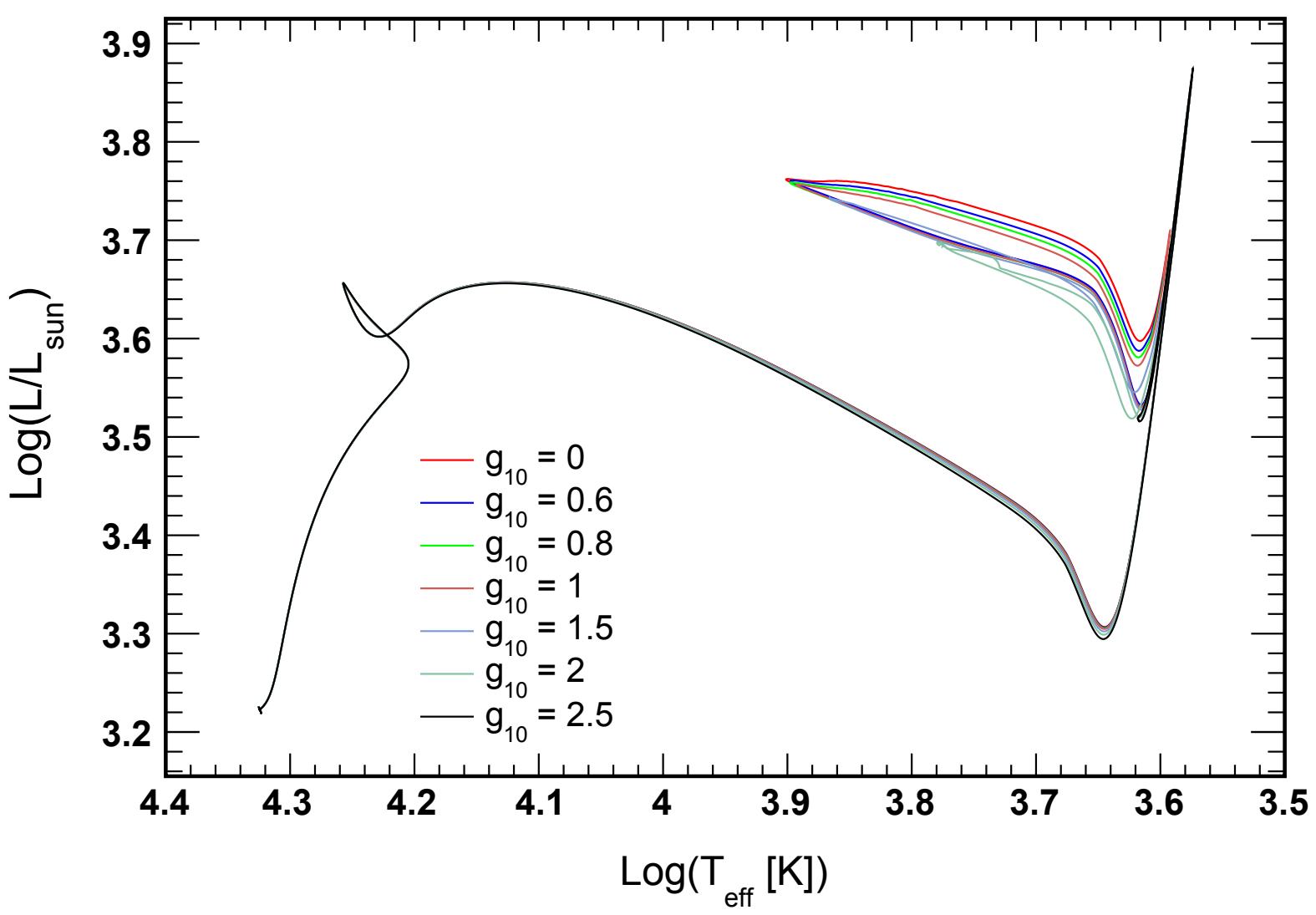

Figura 3.4: Secuencias evolutivas correspondientes al SET1 de parámetros para una estrella de $7 M_{\odot}$ con $g_{10}=0,0.6,0.8,1,1.5,2$ y 2.5 . La desaparición del blue loop ocurre para la secuencia correspondiente a la constante de acoplamiento $g_{10}=2.5$.

De la tabla 3.3, puede evidenciarse que la sensibilidad de los blue loops a la constante de acoplamiento axión-fotón depende de la masa, ya que, para cada uno de los escenarios considerados, se obtiene más de un valor para la cota de dicho acoplamiento. Para el SET4, por ejemplo, tanto en el caso de una masa de $6 M_{\odot}$ como de $7 M_{\odot}$ se obtiene 


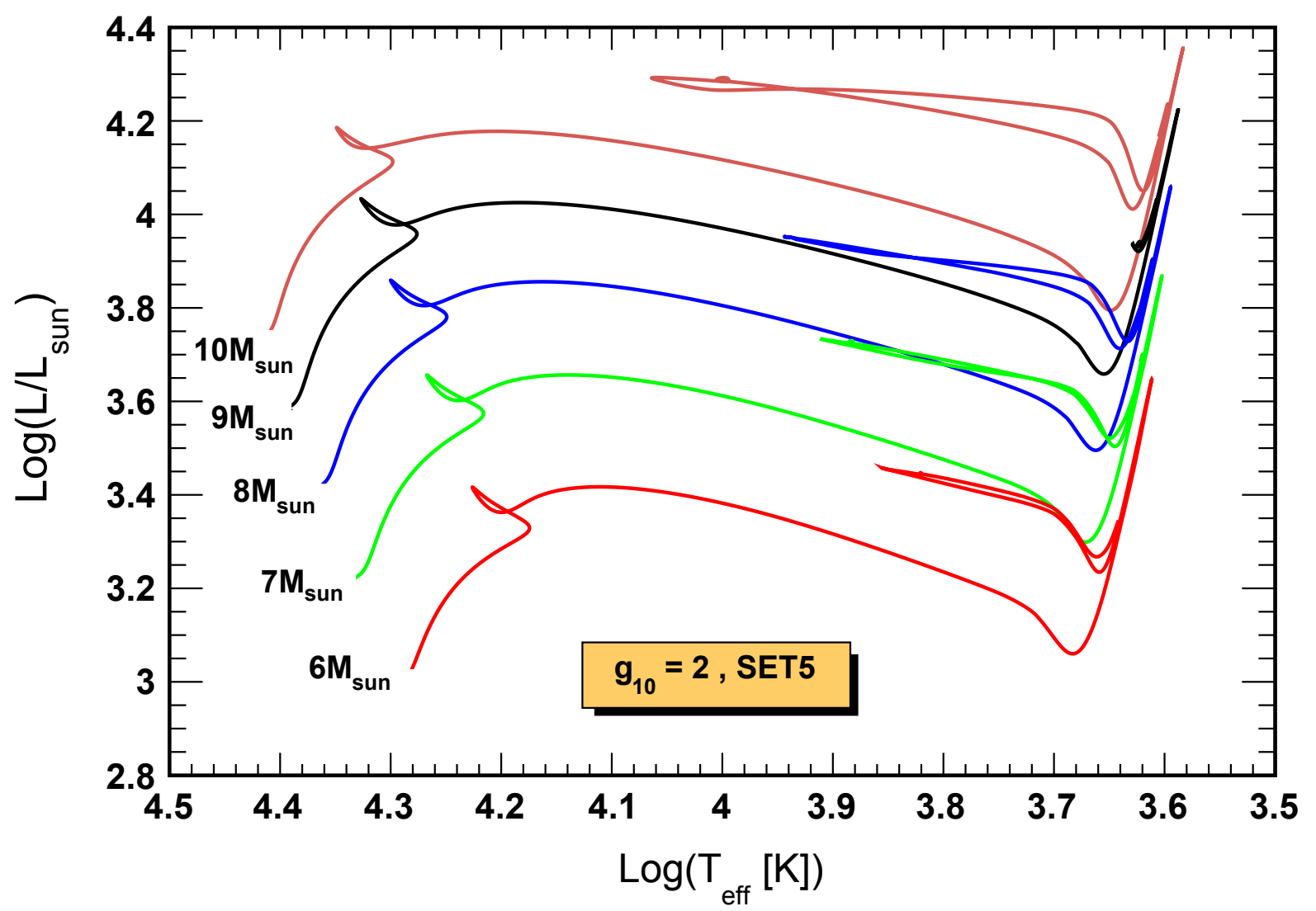

Figura 3.5: Evolución en el diagrama $\mathrm{H}-\mathrm{R}$ para estrellas en el rango $6 M_{\odot}-10 M_{\odot}$ para el SET5 de parámetros con el agregado de un enfriamiento anómalo correspondiente a $g_{10}=2$.

una cota de 0.6 , mientras que si se considera una masa de $9 M_{\odot}$ o $10 M_{\odot}$ la cota se relaja a 2. Por otra parte, la comparación de las distintas columnas de la tabla 3.3 pone de manifiesto la degradación de la cota para $g_{10}$ debido a cambios en los parámetros que determinan la evolución estelar. En efecto, para el SET4 se obtiene una cota muy restrictiva de 0.6, menor a la obtenida en Friedland et al. (2013). Sin embargo, si se pasa del SET4 al SET1 o al SET3, la cota se relaja significativamente al valor de 1.5. Finalmente, tanto el SET2 como el SET5 dan lugar a una cota de 2, lo cual implica una degradación todavía mayor. Teniendo en cuenta estos resultados, se concluye que la cota más conservativa es 2 , siendo este valor el que surge de tomar el máximo de entre el conjunto de las cotas correspondientes a cada SET de parámetros. A su vez, la cota obtenida para cada SET se define como el mínimo valor de $g_{10}$ para el cual la fase de blue loop desaparece de la secuencia evolutiva de alguna de las masas consideras. La robustez de esta cota radica en el hecho de que, sin importar qué SET se considere, es decir, sin 
importar como sea realmente el overshooting dentro de los valores estudiados, siempre alguna de las secuencias evolutivas estará en contradicción con las observaciones por no presentar blue loop. Por supuesto, el calificativo de robusta adjudicado a la cota obtenida cabe en el marco de las incertezas consideradas para el overshooting, por ejemplo, una variación del $74 \%$ en el $f_{n u c}$ en el caso de los SETs 1 y 2 . Si las incertezas fuesen aún más grandes, la cota podría degradarse aún más. A modo ilustrativo, se muestra en la figura 3.5 el diagrama de Hertzsprung-Russell obtenido con el SET5 en el caso en que la constante de acoplamiento axión-fotón toma el valor de la cota más conservativa, $g_{10}=2$. La presencia de axiones en el mecanismo de enfriamiento reduce la fase de blue loop, y particularmente, para $9 M_{\odot}$, el loop es removido.

Como ya fue mencionado, la cota más fuerte entre aquellas dadas en la tabla 3.3 es 0.6, valor que se obtiene con el SET4 de parámetros a partir de la desaparición del blue loop de las secuencias evolutivas correspondientes tanto en el caso de $6 M_{\odot}$ como de $7 M_{\odot}$. Esta cota resulta aún más restrictiva que aquella derivada de estrellas de la rama horizontal en cúmulos globulares $\left(g_{10}<0.66\right)$ (Ayala et al., 2014), ver sección 2.5.1 y está más allá del límite de sensibilidad del experimento CAST $\left(g_{10} \sim 0.88\right)$ para masas del axión muy pequeñas $\left(m_{a}<0.02 \mathrm{eV}\right)$. Sin embargo, como se mencionó anteriormente, la cota más robusta que se desprende de la tabla 3.3 es $g_{10}<2$, la cual se traduce, utilizando la ec. (2.13) con $E / N=0$ (modelo KSVZ) y $z=0.56$, a $m_{a} \lesssim 0.5 \mathrm{eV}$. En este caso, la cota está contenida en aquella obtenida mediante estrellas de la rama horizontal. Esta misma región del espacio de parámetros está siendo también explorada por el helioscopio CAST, el cual mediante el uso de ${ }^{3} \mathrm{He}$ a fin de poder alcanzar presiones más grandes a temperaturas criogénicas, ha logrado imponer límites hasta $1.17 \mathrm{eV}$, los cuales empiezan a traspasar la línea axiónica para el modelo KSVZ, ver figura 2.4. En el futuro, es esperable que la combinación de las búsquedas con Helioscopios y Telescopios confirme la exclusión de la línea correspondiente a axiones KSVZ para masas del axión por encima de $\sim 0.2 \mathrm{eV}$. 


\section{4 \\ Cotas para el axión derivadas de la WDLF Galáctica}

Como se mencionó en la Introducción, la forma de la WDLF es una herramienta poderosa para verificar la posible existencia de axiones de tipo DFSZ, los cuales fueron descriptos en la sección 2.3.2. En este capítulo se derivan cotas para la masa del axión a partir del computo de nuevas WDLFs teóricas sobre la base de modelos evolutivos de EBs que incorporan la retroalimentación de axiones sobre la estructura térmica de la EB. Los resultados aquí obtenidos han sido presentados en diversos congresos y publicados en revistas internacionales (Miller Bertolami et al., 2014; Melendez et al., 2014; Miller Bertolami et al., 2015). Más aún, en el presente capítulo se muestra que la mencionada retroalimentación no puede ser ignorada a altas luminosidades $\left(M_{B o l} \leq 8\right)$. Dichas WDLFs teóricas serán comparadas cuantitativamente con 5 diferentes WDLFs observadas mediante la aplicación del test de $\chi^{2}$. Mientras todas las WDLFs estudiadas en esta Tesis no favorecen masas del axión en el rango sugerido por la astrosismología $\left(m_{a} \cos ^{2} \beta \geq 10 \mathrm{meV} ; g_{a e} \geq 2.8 \times 10^{-13}\right)$, masas del axión más bajas no pueden ser rechazadas con el conocimiento actual de la WDLF del disco Galáctico. 


\subsection{FísicA DE ENTRAdA Y MOdElos IniCiales DE EBS}

Las secuencias evolutivas presentadas en esta Tesis han sido computadas con el código de evolución estelar LPCODE descripto en el apéndice A. Este código fue utilizado también para calcular las secuencias evolutivas de las estrellas Cefeidas presentadas en el capítulo 3. A continuación se listan los principales ingredientes físicos incluidos en las simulaciones numéricas de las EBs. La ecuación de estado para el régimen de alta densidad es la de Segretain et al. (1994), mientras que para el régimen de baja densidad se ha utilizado una versión actualizada de la ecuación de estado de Magni \& Mazzitelli (1979) (Mazzitelli 1993, comunicación privada). Las opacidades conductivas se encuentran en Cassisi et al. (2007), mientras que las opacidades radiativas son las del proyecto OPAL, ver Iglesias \& Rogers (1996), cuyos valores se complementan a bajas temperaturas por las opacidades moleculares descriptas en Ferguson et al. (2005). En el LPCODE, condiciones de bordes para las secuencias evolutivas son provistas por modelos de atmósfera no gris teniendo en cuenta efectos no ideales en la ecuación de estado del gas, ver Rohrmann et al. (2012). El enfriamiento por neutrinos a través de los procesos Bremsstrahlung, foto producción y producción de pares se ha incorporado siguiendo la receta de Itoh et al. (1996). Para los procesos de plasma, en cambio, se siguieron las prescripciones de Haft et al. (1994). Además, todas las fuentes de energía relevantes han sido consideradas en las simulaciones, incluyendo la quema nuclear marginal, la liberación de calor latente y la energía gravitacional asociada con la separación de fase en el perfil de $\mathrm{C}-\mathrm{O}$ inducido por cristalización. En el LPCODE estas fuentes de energía están incorporadas de manera consistente y acopladas localmente al conjunto completo de ecuaciones de evolución estelar. Asimismo, se han tenido en cuenta los efectos de la difusión de elementos siguiendo el tratamiento de Burgers (1969) para gases multicomponente. Vale la pena notar que el LPCODE se ha comparado con otros códigos de evolución estelar conocidos, encontrándose que las incertezas en los tiempos de enfriamiento de las EBs están por debajo del 2\%, ver Salaris et al. (2013). Esto último aporta una base sólida para el estudio desarrollado en este capítulo.

Los modelos iniciales de EBs que se han adoptado en nuestras simulaciones fueron tomados de Renedo et al. (2010). Específicamente, se consideraron 4 modelos iniciales 
de EBs de $0.524 M_{\odot}, 0.609 M_{\odot}, 0.705 M_{\odot}$ y $0.877 M_{\odot}$. Estas secuencias evolutivas fueron obtenidas a partir de computar la evolución completa de progenitores de $1 M_{\odot}, 2 M_{\odot}$, $3 M_{\odot}$ y $5 M_{\odot}$ con $\mathrm{Z}=0.01$, lo que está en acuerdo con las determinaciones semiempíricas de la relación masa inicial-final, ver Salaris et al. (2009).

\subsection{Función De Luminosidad de EBs}

Para construir la función de luminosidad de EBs se ha adoptado el método presentado por Iben \& Laughlin (1989). Es decir, el número de EBs por logaritmo de luminosidad y volumen se ha computado como

$$
\frac{d n}{d l}=-\int_{M_{1}}^{M_{2}} \psi(t)\left(\frac{d N}{d M}\right)\left(\frac{\partial t_{c}}{\partial t}\right)_{m} d M
$$

donde $\psi(t)$ es la tasa de formación estelar galáctica al tiempo $t, N(M)$ es la función de masa inicial y $t_{c}(l, m)$ es el tiempo que transcurre desde la formación de la EB, de masa $m$, hasta que alcanza una luminosidad $l=\log \left(L / L_{\odot}\right)$. Con el objetivo de calcular la integral de la ec. (4.1), se necesitan conocer la relación masa inicial-final $m(M)$ y los tiempos de vida estelares previos a la formación de la $\mathrm{EB}, t_{e v}(M)$. Es necesario destacar que para una dada luminosidad $(l)$ de la EB y masa del progenitor $(M)$, el tiempo de formación de la estrella, $t$, se obtiene resolviendo

$$
t+t_{e v}(M)+t_{c}(l, m)=T_{O S}
$$

donde $T_{O S}$ es la edad de la estrella más vieja en la población computada. La masa inicial más baja que produce una enana blanca con luminosidad $l$ al tiempo presente $\left(M_{1}\right)$ se obtiene de la ec. (4.2) cuando $t=0$. El valor de $M_{2}$ corresponde a la masa del progenitor más grande capaz de producir una EB. Estos conceptos fueron previamente introducidos en la sección 1.3.5.

Con la finalidad de computar el impacto de los axiones sobre la WDLF se han calculado, para cada modelo inicial de EB, 8 secuencias de enfriamiento correspondientes a 
$m_{a} \cos ^{2} \beta=0,2.5,5,7.5,10,15,20$, y $30 \mathrm{meV}$. Asimismo, en la ec. (4.1), se han adoptado la función de masa inicial de Salpeter, la relación masa inicial-final de Salaris et al. (2009), los tiempos de vida de la base de datos del BaSTI, ver Pietrinferni et al. (2004) y una tasa de formación estelar constante.

A los efectos de comparar las secuencias evolutivas con la investigación de Isern et al. (2008), se han cotejado las WDLFs observadas y teóricas dentro del rango $-1 \leq$ $\log \left(L / L_{\odot}\right) \leq-3\left(7.25 \leq M_{B o l} \leq 12.25\right)^{*}$. Asimismo, se ha asumido una edad del disco de 11 Gyr. Con respecto a la normalización de la WDLF teórica, se han tomado dos enfoques distintos. En el primero de ellos se ha seguido el procedimiento de Isern et al. (2008) y se ha normalizado la WDLF de manera tal que, para un dado valor de $l_{b i n}$, se tiene

$$
\frac{d n}{d l}\left(l_{b i n}\right)=n^{O}\left(l_{b i n}\right)
$$

donde $n^{O}\left(l_{b i n}\right)$ representa el número de estrellas por unidad de volumen y por bin de luminosidad de las WDLFs Galácticas presentes en Isern et al. (2008); Harris et al. (2006); De Gennaro et al. (2008) en el bin de luminosidad $\log \left(L / L_{\odot}\right)=l_{\text {bin }}$. En este esquema de normalización, $l_{\text {bin }}$ se ha tomado igual a todos los bines de luminosidad presentes entre $l \simeq-2$ y $l \simeq-3$ (el valor exacto depende del bineado de cada WDLF). Es importante notar que solo puntos de normalización en el rango de bajas luminosidades deberían ser considerados ya que de lo contrario se estaría normalizando justo en el rango de luminosidad donde los axiones son un mecanismo de enfriamiento dominante y entonces los efectos diferenciales de la emisión de axiones quedarían ocultos. Como se verá en la sección 4.3, la elección de un punto específico de normalización no afecta significativamente las conclusiones principales que se siguen de este capítulo, aunque sí tiene algún impacto en la comparación cuantitativa entre la WDLF teórica y observada. Con la finalidad de que las WDLFs sea menos dependientes del punto de normalización elegido, se adoptó un segundo esquema de normalización. Específicamente, se ha normalizado la WDLF imponiendo que el número total de estrellas por unidad de volumen en un dado rango de magnitud $\left(M_{B o l}^{1}, M_{B o l}^{2}\right)$ ajuste a las observaciones, es decir,

\footnotetext{
* En esta Tesis la relación entre la magnitud bolométrica y la luminosidad de la estrella se ha tomado consistentemente con los datos de Harris et al. (2006), es decir, $M_{B o l}=-2.5 \log \left(L / L_{\odot}\right)+4.75$
} 


$$
\sum_{M_{B o l}^{i} \epsilon\left(M_{B o l}^{1}, M_{B o l}^{2}\right)} n^{O}\left(M_{B o l}^{i}\right) \Delta M_{B o l}=\int_{M_{B o l}^{1}}^{M_{B o l}^{2}} \frac{d n}{d l} d M_{B o l} .
$$

Para los límites de integración $\left(M_{B o l}^{1}, M_{B o l}^{2}\right)$ se ha tomado $(9.5,12.5)$ y $(9.6,12.4)$ para las WDLFs presentes en (Harris et al. (2006); Isern et al. (2008); Rowell \& Hambly (2011)) y (Miller Bertolami (2014); De Gennaro et al. (2008)), respectivamente.

\subsection{IMPACTO DE LA EMISIÓN DE AXIONES EN EL ENFRIAMIENTO DE LAS EBS}

La figura 4.1 muestra la emisión de axiones, fotones y neutrinos para una EB de $0.609 M_{\odot}$ suponiendo distintas masas para el axión. Es claro de la figura que, para una misma luminosidad, la emisión de axiones lleva a un decrecimiento de la emisión de neutrinos. Precisamente uno de los resultados de los cálculos realizados es que la retroalimentación de la emisión de axiones sobre la emisión de neutrinos no puede ser omitida en el rango de masas sugerido por determinaciones astrosismológicas $\left(m_{a}>10 \mathrm{meV}\right)$ si se consideran luminosidades relativamente altas para la $\mathrm{EB}\left(M_{b o l} \simeq 8\right)$. Este resultado se debe al hecho de que al incluir la emisión de axiones se provoca un enfriamiento extra del núcleo de la EB, lo que altera la estructura térmica de la misma respecto del caso sin emisión de axiones. A su vez, esto conduce a un decrecimiento de la emisión de neutrinos para una dada luminosidad superficial de la estrella. La principal consecuencia que se sigue de ello es que el enfriamiento de la EB resulta menos sensible a la existencia de axiones de lo que un enfoque perturbativo podría sugerir, ya que la pérdida de energía adicional debida a la existencia de axiones es contrabalanceada por el decrecimiento de la energía perdida por la emisión de neutrinos. Es preciso notar que la emisión de neutrinos se ve afectada por la emisión de axiones para masas incluso tan pequeñas como $m_{a} \cos ^{2} \beta=5 \mathrm{meV}$. En consecuencia, la emisión de axiones debe ser tratada de manera autoconsistente para altas luminosidades $\left(M_{b o l} \simeq 8\right)$ cuando se trata con axiones en el rango de la astrosismología o en el rango detectable a través de la WDLF. Para bajas luminosidades $\left(M_{b o l} \geq 8\right)$, en cambio, la emisión de neutrinos es despreciable y la velocidad de enfriamiento no se encuentra afectada por la retroalimentación de la emisión de axiones. Claramente, a bajas luminosidades la retroalimentación de la emisión de axiones en la energía total de la estrella es despreciable. 


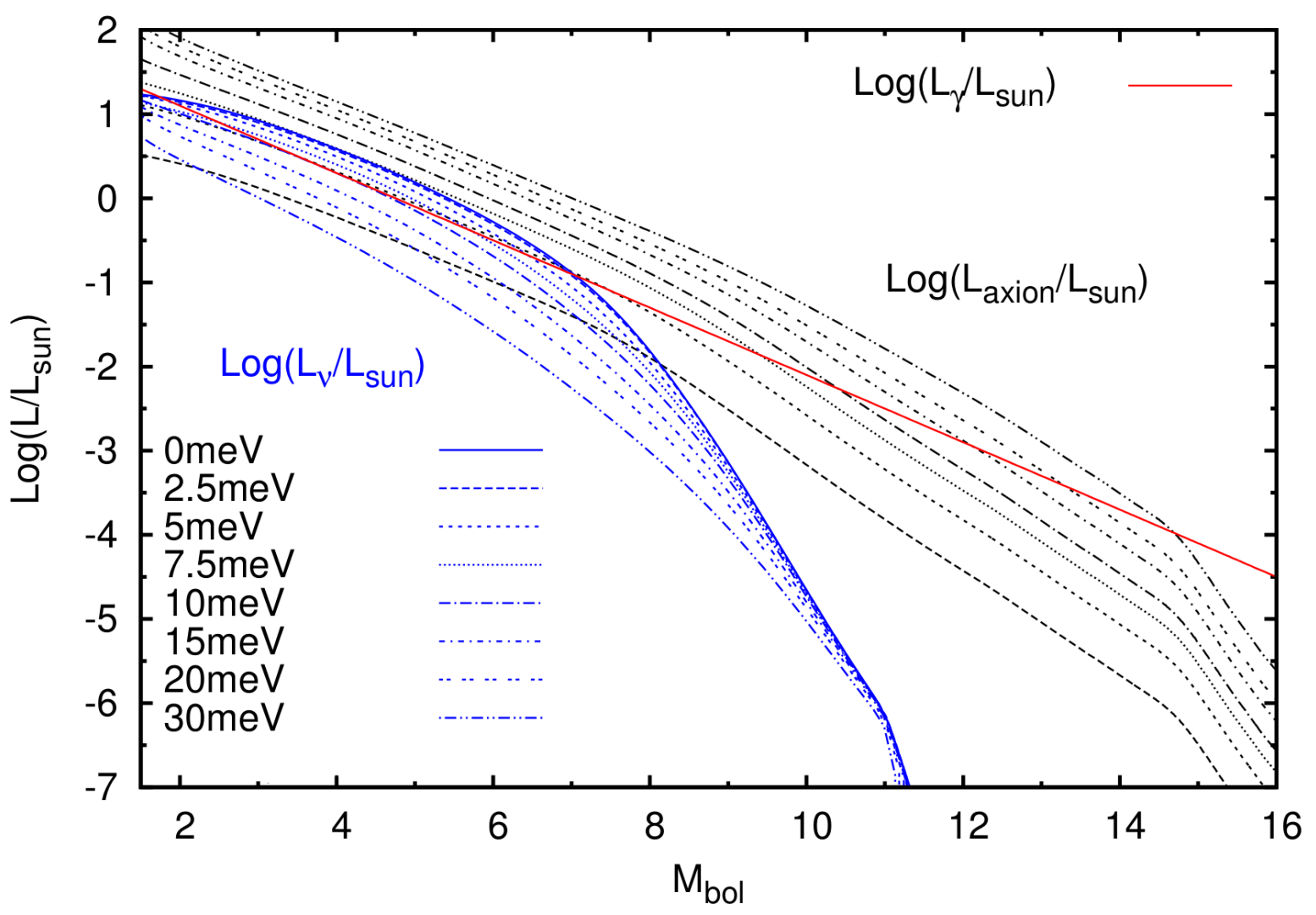

Figura 4.1: Emisión de fotones (línea roja), axiones (curvas negras) y de neutrinos (curvas azules) para la secuencia de $0.609 M_{\odot}$ asumiendo distintas masas para el axión. El impacto de la emisión de axiones en la estructura térmica de la EB se evidencia en el decrecimiento de la emisión de neutrinos para altas luminosidades. Claramente, la emisión de axiones no puede ser tratada de manera perturbativa para $m_{a} \cos ^{2} \beta>5 \mathrm{meV}$.

En la figura 4.2 se comparan la emisión de axiones y neutrinos de la secuencia correspondiente a $0.609 M_{\odot}$ con la secuencia correspondiente a $0.601 M_{\odot}$ de Isern et al. (2008) para un axión de $m_{a} \cos ^{2} \beta=5 \mathrm{meV}$. Es importante destacar que existe un buen acuerdo para la emisión de axiones entre ambas predicciones a bajas luminosidades $\left(M_{b o l} \geq 7\right)$. El apartamiento entre ambas curvas a altas luminosidades $\left(M_{b o l} \leq 7\right)$ proviene de la aproximación de núcleo isotérmico de Isern et al. (2008), la cual lleva a una subestimación de la emisión de axiones a altas luminosidades cuando la temperatura máxima del núcleo no se da en el centro del mismo. Además, como se mencionó anteriormente, para obtener escalas de tiempo precisas la retroalimentación de la emisión de axiones en la estructura térmica de la EB es significativa únicamente para altas luminosidades $\left(M_{b o l} \leq 8\right)$ y, por lo tanto, de escasa relevancia para el rango de luminosidades estudiado 
en la mayor parte de este capítulo $\left(7 \leq M_{B o l} \leq 12.5\right)$. Consecuentemente, en lo que hace a la comparación de las WDLFs teórica y observada en el rango de $7 \leq M_{B o l} \leq 12.5$, el impacto de las aproximaciones adoptadas en Isern \& García-Berro (2008) y Isern et al. (2008) puede ser despreciado.

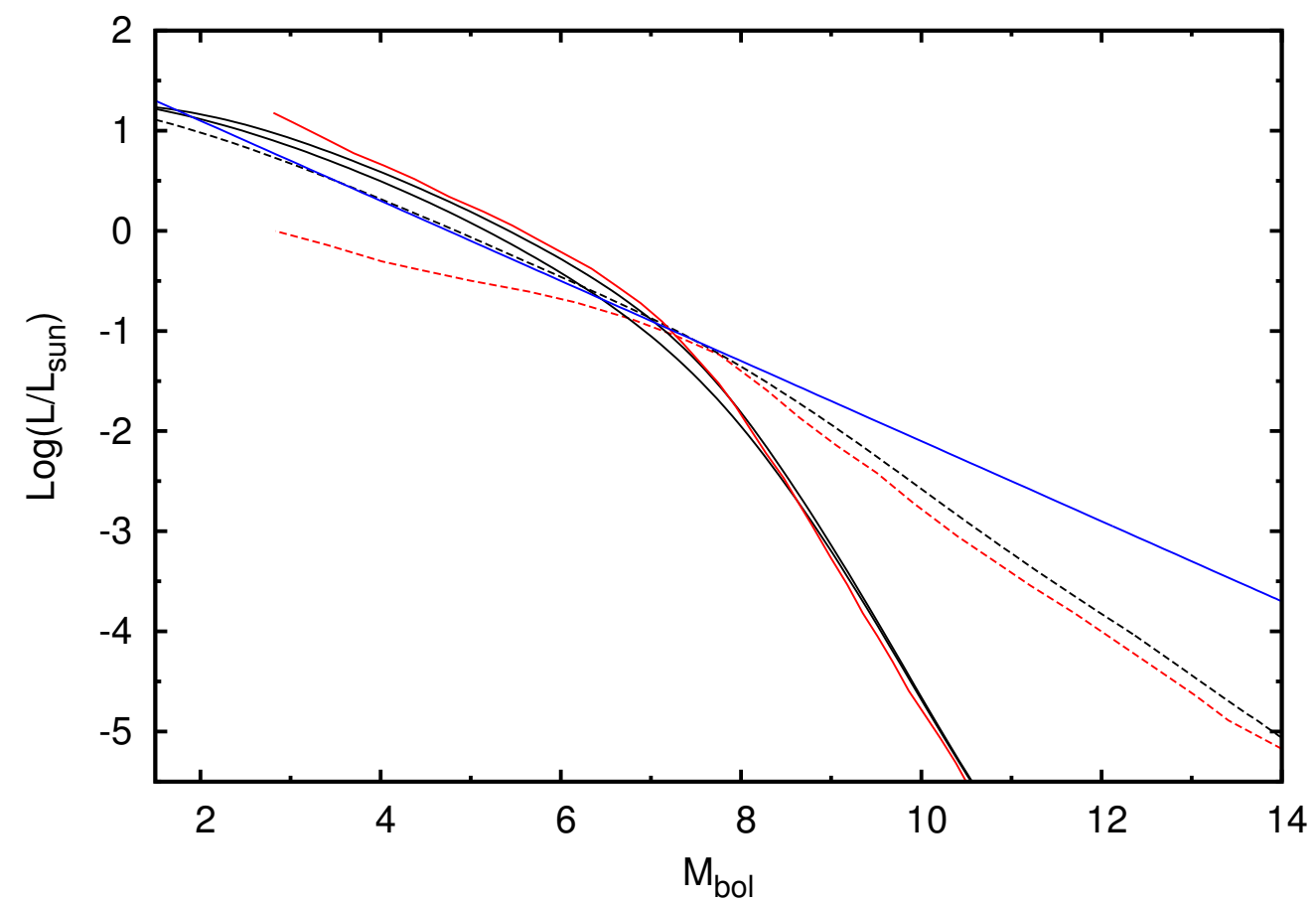

Figura 4.2: Comparación de la emisión de axiones $\left(m_{a} \cos ^{2} \beta=5 \mathrm{meV}\right)$ y la emisión de neutrinos de la secuencia de $0.609 M_{\odot}$ realizada en este trabajo con la secuencia de $0.61 M_{\odot}$ de Isern et al. (2008). La línea sólida negra corresponde a la luminosidad de neutrinos (desde arriba hacia abajo: $m_{a} \cos ^{2} \beta=0,5 \mathrm{meV}$ ) del modelo presentado en este capítulo. La línea sólida roja representa la emisión de neutrinos del modelo de Isern, la línea punteada negra la emisión de axiones para $5 \mathrm{meV}$ de este trabajo y la línea punteada roja la emisión de axiones para $5 \mathrm{meV}$ del modelo de Isern et al. (2008). Finalmente, la línea azul representa la luminosidad de fotones. Los efectos del apartamiento de la aproximación de núcleo isotérmico son apreciables para $M_{b o l}<7$.

\subsection{IMPACTO DE LA EMISIÓN DE AXIONES EN LA WDLF}

Se han considerado cinco WDLF del disco Galáctico con el objetivo de tener en cuenta todos los posibles errores sistemáticos en la comparación y también de contrastar con trabajos previos. Más precisamente, se han comparado las WDLFs teóricas desarrolladas en esta Tesis con aquellas derivadas, o construidas, por Isern et al. (2008); Harris et al. (2006); De Gennaro et al. (2008); Rowell \& Hambly (2011) y Miller Bertolami 
(2014), ver figura 4.3. Mientras que la WDLF de Harris et al. (2006) se obtuvo del SDSS-DR3 mediante la técnica de movimientos propios reducidos sin discriminar entre EBs DA y no DA, la WDLF de De Gennaro et al. (2008) se derivó del SDSS-DR4 considerando únicamente EBs ricas en $\mathrm{H}$ identificadas espectroscópicamente. Con la intención de posibilitar la comparación directa de los resultados obtenidos en este capítulo con aquellos reportados por Isern et al. (2008), también se ha incluido en este estudio la WDLF allí presentada, la cual fue construida a partir del SDSS-DR3 (Harris, Marzo de 2005, comunicación privada) con $V_{\tan }>20 \mathrm{~km} / \mathrm{s}$. Todos los trabajos previos acerca de la WDLF provienen del relevamiento SDSS, por lo que se ha decidido incluir también en el presente análisis la WDLF completamente independiente extraída del relevamiento SuperCOSMOS por Rowell \& Hambly (2011). Finalmente, para hacer uso de las nuevas WDLFs que se extienden al régimen de alta luminosidad, se han incluido también las WDLFs construidas por Miller Bertolami (2014) a partir de dos conjuntos completamente independientes de WDLFs, ver Krzesinski et al. (2009) y Rowell \& Hambly (2011).

\subsubsection{WDLFS PARA LUMINOSIDADES INTERMEDIAS}

En la figura 4.4 se muestran, como ejemplo, las WDLFs resultantes para cada masa del axión junto con la WDLF obtenida por Harris et al. (2006). Claramente, se observa que masas mayores a los $10 \mathrm{meV}$ conducirían a evidentes desacuerdos con la función de luminosidad derivada por Harris et al. (2006). Además, valores grandes para la masa del axión no presentan un buen acuerdo con las WDLFs de De Gennaro et al. (2008), Isern et al. (2008) y Rowell \& Hambly (2011). Para tener una medida cuantitativa del

acuerdo entre la WDLF computada bajo distintas suposiciones y las WDLFs observadas (figura 4.3), se ha realizado un test de $\chi^{2}$. El valor de $\chi^{2}$ se computó de acuerdo a la siguiente expresión:

$$
\chi^{2}\left(m_{a}\right)=\frac{1}{(N-1)} \sum_{i=1}^{N} \frac{\left(n_{i}^{\mathrm{O}}-n_{i}^{m_{a}}\right)^{2}}{\sigma_{i}{ }^{2}}
$$

donde $N=11$ para Harris et al. (2006); Rowell \& Hambly (2011), 13 para De Gennaro et al. (2008) o 10 en el caso de Isern et al. (2008). Asimismo, $n_{i}^{\mathrm{O}}$ denota los valo- 


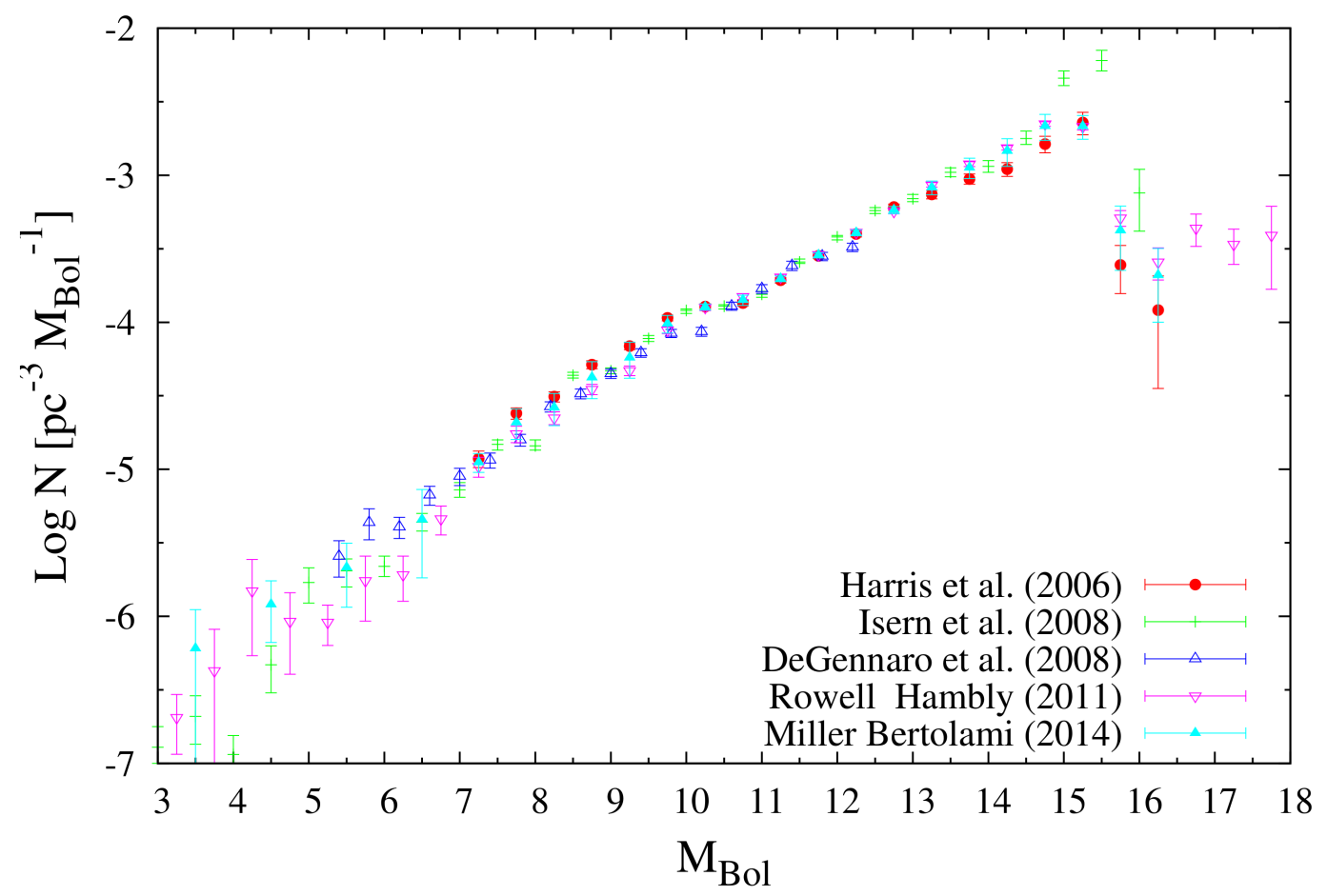

Figura 4.3: WDLFs del disco Galáctico adoptadas a fines comparativos. La WDLF de Rowell \& Hambly (2011) ha sido multiplicada por un factor de 1.862 como se describe en Miller Bertolami (2014).

res derivados en cada WDLF observacional y $n_{i}^{m_{a}}$ representa la predicción teórica del número de estrellas bajo la suposición de distintas masas del axión $\left(m_{a}\right)$. Las cantidades $\sigma_{i}^{2}$ se han estimado como aquellos errores reportados para cada WDLF observacional, es decir, se han despreciado las posibles incertezas provenientes de la teoría de evolución estelar. El factor $(N-1)$ en la ec. (4.5) toma en cuenta el hecho de que los valores $n_{i}^{m_{a}}$ no son completamente independientes de las observaciones sino que han sido normalizados para ajustar a las mismas, como se describe en las ec. (4.3) y ec. (4.4). Los valores de $\chi^{2}$ obtenidos de la ec. (4.5) son, en todos los casos, demasiado grandes, lo que implica que existe un desacuerdo significativo entre todas las WDLFs teóricas y las observadas. Este desacuerdo puede adjudicarse a dos causas. Por un lado, las distintas WDLFs no son consistentes entre ellas, sugiriendo que las incertezas son más grandes que las efectivamente reportadas. En particular, fluctuaciones en la tasa de formación estelar (SFR) de la Galaxia podrían introducir apartamientos apreciables de la hipótesis de una SFR constante. Afortunadamente, aunque los valores derivados del test de $\chi^{2}$ 


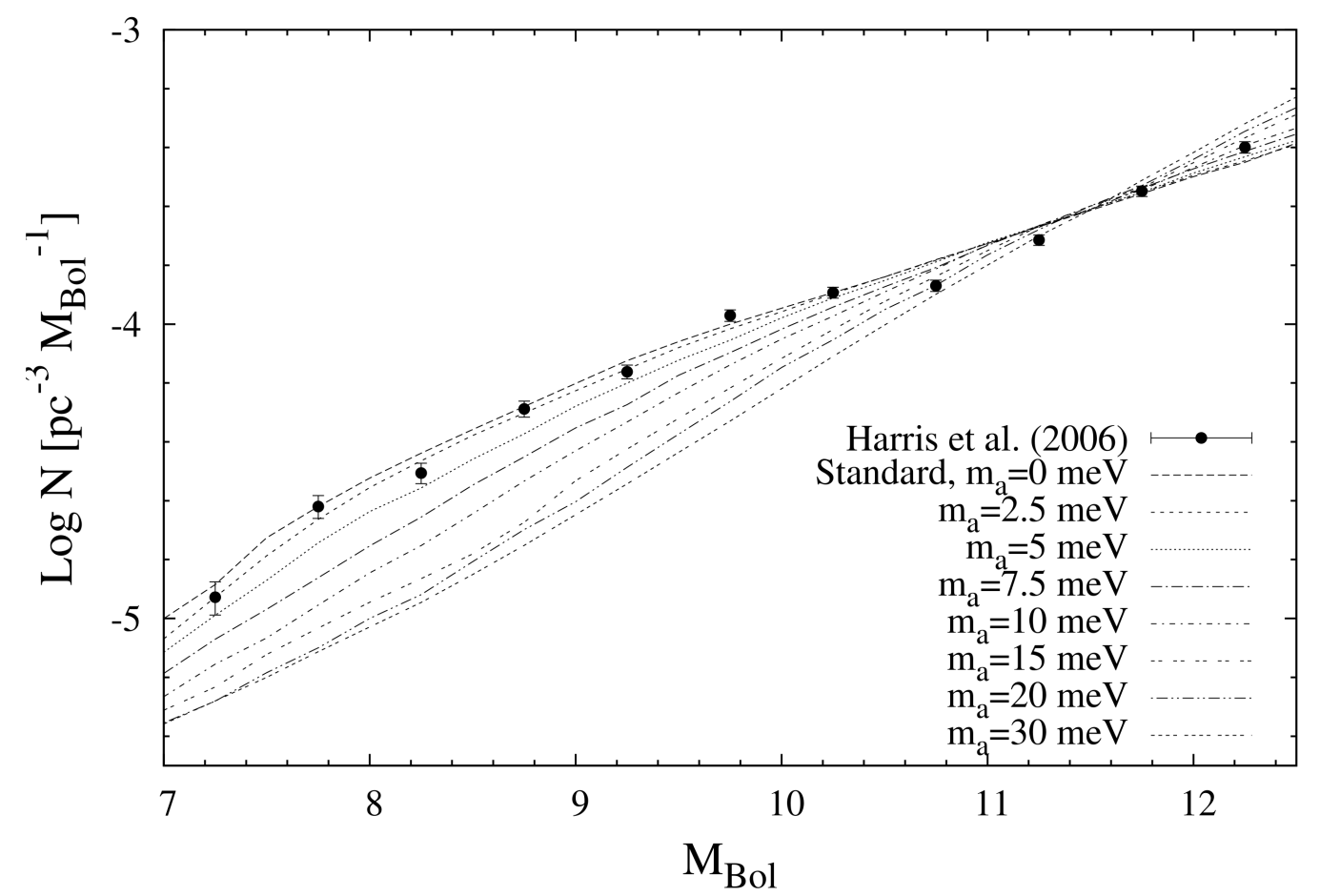

Figura 4.4: WDLFs construidas para distintas masas del axión comparadas con la WDLF de Harris et al. (2006). Los axiones DFSZ con $m_{a}>10 \mathrm{meV}$ están claramente excluidos por la WDLF observada. Es posible notar que las WDLF teóricas construidas con $m_{a}>10 \mathrm{meV}$ están casi superpuestas en el rango de interés. Esto se debe al hecho de que la región de normalización es, en esos casos, también afectada por la emisión de axiones.

son grandes, existen diferencias significativas, de más de un orden de magnitud, entre el mejor ajuste de $\chi^{2}$ y el resto.

En la figura 4.5 se muestran, para cada WDLF, los valores de $\chi^{2}$ respecto de su valor mínimo para cada punto de normalización. Como puede observarse de dicha figura, mientras que la WDLF de Harris et al. (2006) prefiere valores para la masa del axión $m_{a} \cos ^{2} \beta \leq 2.5 \mathrm{meV}$, las WDLFs de De Gennaro et al. (2008) y Rowell \& Hambly (2011) favorecen la existencia de un enfriamiento extra en el rango de $5 \mathrm{eV} \leq \mathrm{m}_{\mathrm{a}} \cos ^{2} \beta \leq$ $7.5 \mathrm{meV}$. Es preciso notar que la naturaleza de este resultado es independiente del punto de normalización o del método de normalización de la WDLF teórica. De hecho, cuando se adopta el segundo método de normalización para la WDLF (ec. 4.4), se reobtiene el mismo resultado global. El hecho de que las distintas WDLFs favorezcan distintos rangos para la masa del axión era de esperarse si se tiene en cuenta que las diferencias entre las mismas exceden las barras de error, ver figura 4.3. Asimismo, cuando se compara la 
WDLF de Isern et al. (2008) (la cual es una versión preliminar de Harris et al. (2006)), se reobtienen los principales resultados citados en tal artículo, es decir, el mejor ajuste corresponde a masas del axión en el rango $2.5 \mathrm{meV} \leq \mathrm{m}_{\mathrm{a}} \cos ^{2} \beta \leq 7.5 \mathrm{meV}$ dependiendo del punto o método de normalización. Se debe notar, sin embargo, que en este caso las diferencias en los valores de $\chi^{2}$ son más pequeñas que en los tres casos anteriores y entonces límites menos restrictivos pueden ser impuestos.
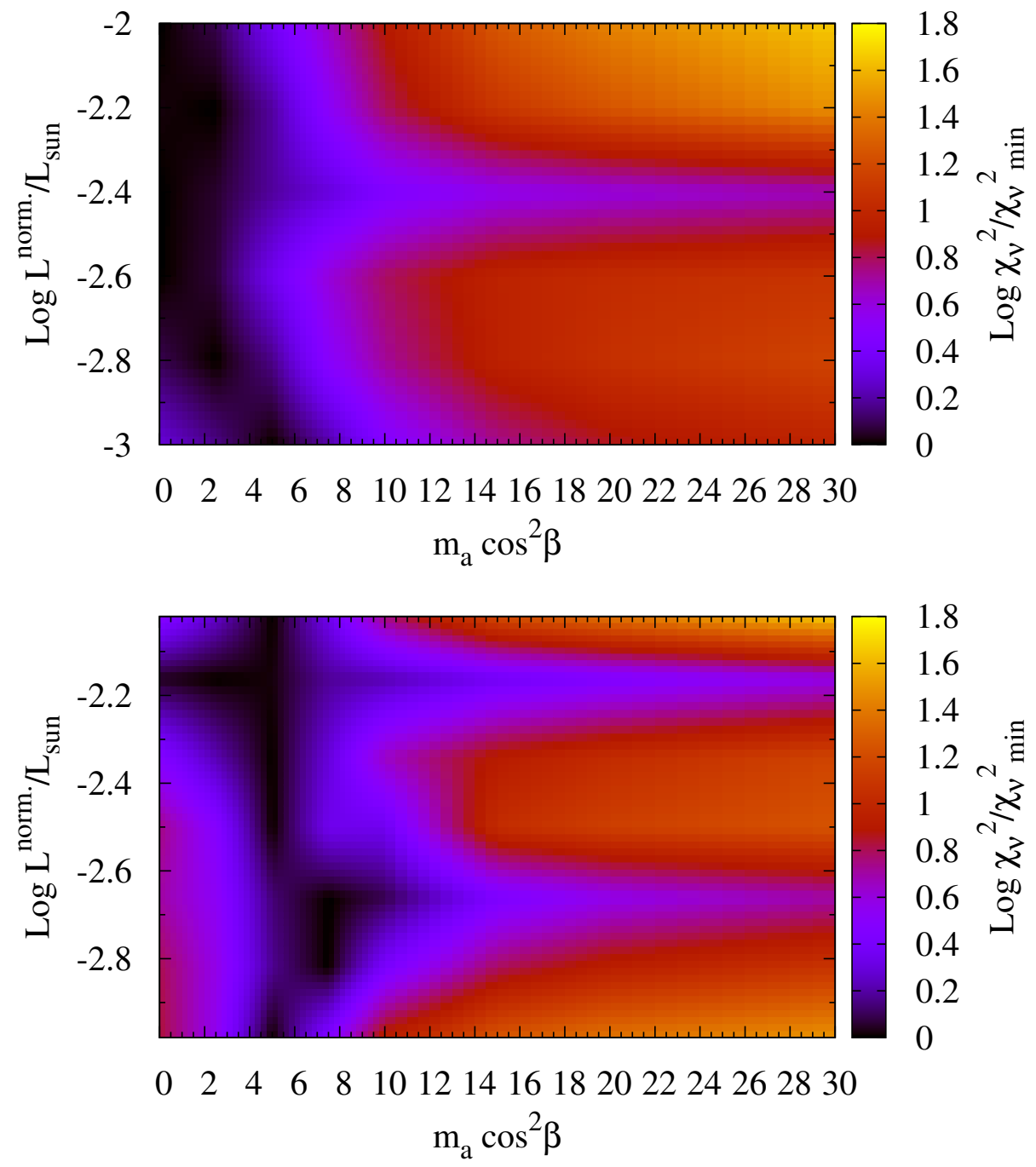

Figura 4.5: Valores de $\chi^{2}$ (mapa de color) derivados de la comparación de las WDLFs teóricas con aquellas obtenidas por (de arriba hacia abajo) Harris et al. (2006), De Gennaro et al. (2008), Rowell \& Hambly (2011) y Isern et al. (2008) para distintas elecciones del punto de normalización (eje y) y la masa del axión $($ eje $x)$. 

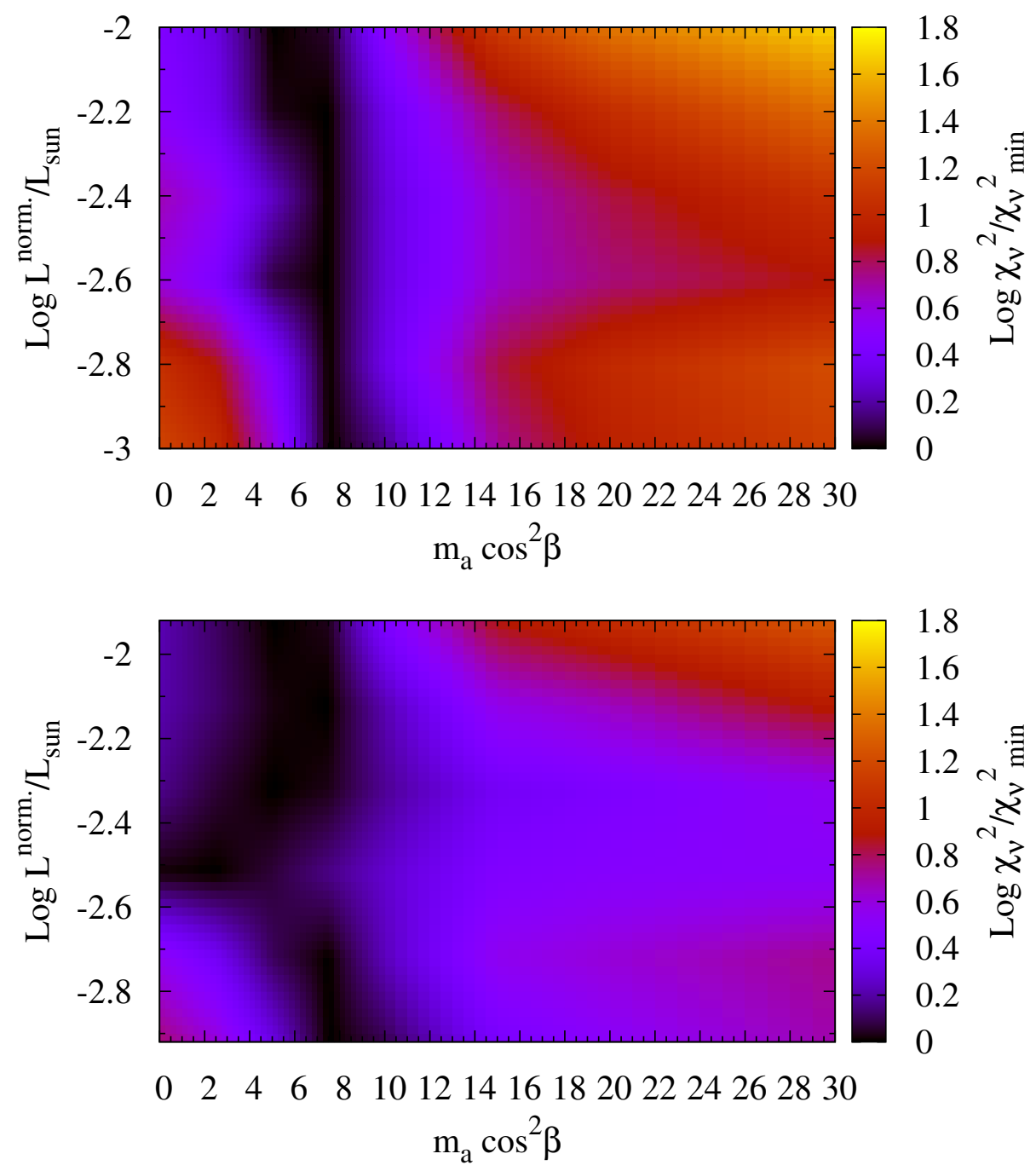

Figura 4.5: Continuación

\subsubsection{Cotas Derivadas a PARTIR DE LA WDLF EN EL RANGo $3<M_{b o l}<12.5$}

La figura 4.6 muestra la comparación entre la WDLF computada para distintas masas del axión con la WDLF del disco Galáctico derivada por Miller Bertolami (2014). Esta WDLF se construyó a partir de la WDLFs determinadas por los relevamientos SDSS (Harris et al. (2006); Krzesinski et al. (2009)) y SuperCOSMOS (Rowell \& Hambly (2011)). El tamaño de las barras de error refleja las discrepancias entre ambas WDLFs. De esta manera, las barras de error no muestran solamente incertezas estadísticas internas sino que también describen discrepancias sistemáticas entre ambas WDLFs, ver 
Miller Bertolami (2014) para una discusión detallada acerca de estas cuestiones. Asimismo, esta WDLF incluye información para luminosidades muy altas donde se espera que los axiones sean una fuente importante de enfriamiento aún para masas pequeñas. Como en los casos anteriores, se han comparado las WDLFs teóricas y observadas adoptando distintos puntos de normalización y métodos (ec. 4.3 y ec. 4.4). Al igual que en los casos anteriores (ver figura 4.5), para el primer método de normalización la WDLF teórica que mejor ajusta depende ligeramente del punto de normalización escogido, pero el principal resultado es estable: el test de $\chi^{2}$ prefiere valores para la masa del axión en el rango de $m_{a} \leq 5 \mathrm{meV}$, mientras que masas del axión $m_{a} \gtrsim 10 \mathrm{meV}$ son excluidas con un alto nivel de confianza. Esto se puede observar en la figura 4.6, donde se muestran los resultados para el caso del segundo método de normalización. Como se puede apreciar en el recuadro interno de dicha figura, el modelo que mejor ajusta corresponde a $m_{a} \cos ^{2} \beta \sim 5 \mathrm{meV}$ y modelos con $m_{a} \cos ^{2} \beta \leq 7.5 \mathrm{meV}$ no pueden ser rechazados concluyentemente. Por el contrario, todas las WDLF construidas con $m_{a} \cos ^{2} \beta \geq 10 \mathrm{eV}$ están en claro desacuerdo con la WDLF de Miller Bertolami (2014). 


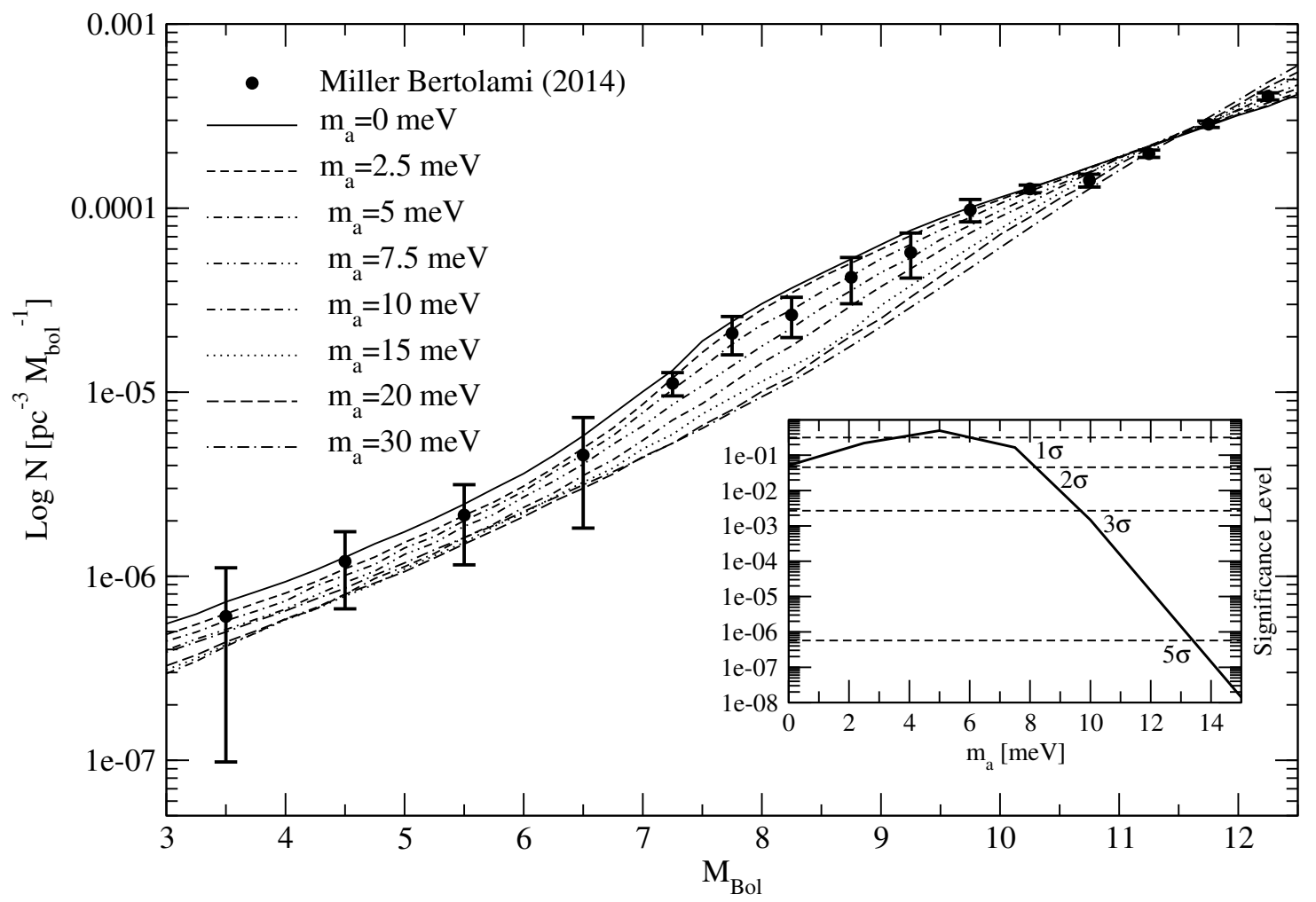

Figura 4.6: Comparación de la WDLF construida por Miller Bertolami (2014) con la WDLF teórica para distintas masas del axión. La línea continua en el recuadro interior muestra el nivel de confianza con el cual los distintos valores de la masa del axión son descartados por el test $\chi^{2}$. Por referencia se indican mediante líneas discontinuas los niveles de significancia asociados con diferentes valores de $\sigma$. 


\section{5 Conclusiones y Trabajo a Futuro}

En esta Tesis se ha estudiado la posibilidad de utilizar las poblaciones estelares como laboratorios de física fundamental, haciendo foco en las propiedades de la partícula elemental denominada axión. La elección del axión como la partícula a estudiar tiene la doble motivación de que, permite dar una solución al problema fuerte de CargaParidad (CP) a la vez que brinda un candidato natural a materia oscura. En particular, se ha analizado el impacto producido por la existencia del axión sobre las propiedades observables de estrellas variables de tipo Cefeidas Clásicas y de Enanas Blancas con el objetivo de acotar las constantes de acoplamiento $g_{a \gamma \gamma}$ y $g_{a e}$, respectivamente.

En el capítulo 3 se ha estudiado la emisión de axiones de tipo KSVZ por efecto Primakoff en el interior de estrellas Cefeidas Clásicas. Más precisamente, se ha extendido el trabajo de Friedland et al. (2013) teniendo en cuenta la influencia que sobre la cota para $g_{a \gamma \gamma}$ pueden ejercer las incertezas existentes en los modelos de estructura y evolución estelar. A fin de cuantificar hasta que punto la cota sobre $g_{a \gamma \gamma}$ se degrada al variar la física de entrada y, a su vez, obtener una cota robusta, se han computado 160 simulaciones numéricas que involucran estrellas en el rango de masas $6 \mathrm{M}_{\odot}-10 \mathrm{M}_{\odot}$, cinco escenarios distintos para los parámetros de overshooting en el núcleo y en la envoltura, 
y los siguientes valores para el acoplamiento axión-fotón: 0, 0.6, 0.8, 1, 1.5, 2, 2.5, y 3. Para cada masa y cada uno de los escenarios correspondientes a la física de entrada considerados, se obtuvo una cota para $g_{10}$ requiriendo la desaparición de la fase de blue loop en las secuencia evolutivas.

La primera conclusión que se desprende de los resultados obtenidos es que la sensibilidad de las Cefeidas a la constante de acoplamiento axión-fotón $g_{a \gamma \gamma}$ depende de la masa. En efecto, para cada uno de los escenarios, se han obtenido múltiples valores para la cota de dicho acoplamiento. Por ejemplo, para el SET4, el cual corresponde a $f_{n u c}=0$ y $f_{\text {env }}=0.01$, se ha extraído una cota de 0.6 tanto en el caso de una masa de $6 M_{\odot}$ y de $7 M_{\odot}$, mientras que usando las secuencias evolutivas asociadas a las estrellas de $9 M_{\odot}$ y $10 M_{\odot}$ se ha arribado a una cota de 2. Por tanto, si bien la contribución de axiones tiene siempre el efecto de acortar la fase de blue loop, el factor con el que dicha fase se reduce respecto al caso estándar presenta una dependencia con la masa de la estrella considerada. De hecho, el ajuste de $t_{\mathrm{He}} / t_{\mathrm{He}}\left(g_{10}=0\right)$ con la forma funcional $1 /\left(1+A g_{10}^{2}\right)$ da lugar a una variación de casi el $27 \%$ para el parámetro $A$ cuando se considera el rango de masas $6 M_{\odot}-10 M_{\odot}$. Esto estaría en contraposición con Friedland et al. (2013), donde se afirma que la fase de quema de He se acelera en un factor universal.

Para cada uno de los cinco escenarios o SETs supuestos, se ha obtenido una única cota definida como el mínimo valor de $g_{10}$ para el cual la fase de blue loop desaparece de la secuencia evolutiva de alguna de las masas consideras. Así, para los SETs del 1 al 5 se obtuvieron las cotas: 1.5, 2, 1.5, 0.6, 2, respectivamente. Estos resultados ponen de manifiesto la degradación de la cota para $g_{10}$ que se produce al cambiar los parámetros de overshooting. Mientras que para el SET4 se obtiene la cota más restrictiva (0.6), la cual es incluso menor a la obtenida en Friedland et al. (2013) (0.88) y a la correspondiente a estudios de estrellas de la rama horizontal en cúmulos globulares (0.66) (Ayala et al., 2014). Sin embargo, en el caso de los SETs 2 y 5 la cota se degrada a 2. Teniendo en cuenta las cotas obtenidas para cada uno de los SETs de parámetros considerados, se concluye que la cota más conservativa es 2, la cual queda entonces contenida en aquella derivada utilizando estrellas de la rama horizontal. La robustez de la cota aquí obtenida proviene del hecho de que, sin importar cómo sea realmente el overshooting 
dentro de los valores estudiados, siempre alguna de las secuencias evolutivas estará en contradicción con las observaciones por no presentar la fase de blue loop.

La solidez de la cota global establecida $\left(g_{10}<2\right)$ debe entenderse en el contexto de las incertezas consideradas para el overshooting. Si las incertezas fuesen aún mayores, la cota podría relajarse más todavía. Por tanto, a fin de establecer una cota definitiva para $g_{10}$ por medio de la aplicación del argumento de Friedland et al. (2013) en estrellas Cefeidas, es necesario un estudio minucioso de la convección estelar y una determinación precisa de las incertezas asociadas.

Debido a que se ha hecho uso del criterio propuesto por Friedland et al. (2013) para acotar la constante de acoplamiento axión-fotón, todas las cotas reportadas en el capítulo 3 surgen de requerir la completa eliminación del blue loop, es decir, la desaparición de la población entera de estrellas Cefeidas. Si bien este es el criterio más conservativo, la cota podría refinarse estudiando si el número de estrellas en la fase de blue loop se ve reducido. Por ejemplo, podría compararse el número de estrellas Cefeidas en el rango $6 M_{\odot}-10 M_{\odot}$ reportadas en la base de datos de "David Dunlap Observatory", ver Fernie et al. (1995), con el obtenido de las simulaciones computadas en el capítulo 3 para los distintos acoplamientos considerados. Este último número podría obtenerse, a su vez, extrayendo el tiempo de permanencia en la banda de inestabilidad de cada una de las secuencias simuladas, ya que el número de estrellas de una cierta masa resulta proporcional a dicho tiempo. Este tipo de estudio sería una extensión interesante de lo hecho en el capítulo 3 y podría dar lugar a una cota más competitiva con aquella derivada en Ayala et al. (2014).

Con la finalidad de poner cotas a la constante de acoplamiento entre axiones y electrones $\left(g_{a e}\right)$, en el capítulo 4 se ha realizado un estudio detallado del impacto de la emisión de axiones sobre el enfriamiento de las EBs. En particular, se han mejorado trabajos previos mediante la inclusión de un tratamiento autoconsistente de la emisión de axiones en la estructura térmica de los modelos de EBs y, por otra parte, se han incorporado distintas WDLFs en el análisis. Más precisamente, por primera vez, se ha extendido la comparación entre las WDLFs teóricas y observacionales al régimen de alta luminosidad, donde se espera que los axiones sean el principal mecanismo de en- 
friamiento. Esto último se ha realizado sobre la base de los modelos de EBs y microfísica más avanzados disponibles en la actualidad. Asimismo, se ha testeado la dependencia de la mencionada comparación con la elección de los puntos de normalización de las WDLFs teóricas, así como también con los métodos de normalización adoptados. Por otra parte, se ha ponderado el acuerdo entre la teoría y las observaciones por medio del test de $\chi^{2}$.

Los principales resultados obtenidos en el capítulo 4 concernientes a las EBs y a los axiones DFSZ pueden resumirse como sigue. En el rango de luminosidades $7 \leq M_{B o l} \leq$ 12.5, se encuentra un buen acuerdo entre el tratamiento perturbativo adoptado por Isern et al. (2008) y el resultado de los cálculos evolutivos completos autoconsistentes presentados en esta Tesis. Sin embargo, para el rango $M_{B o l} \leq 7$, tanto la retroalimentación sobre la estructura térmica de la EB y el apartamiento de la aproximación de núcleo isotérmico deben ser considerados, más aún a la luz de las nuevas determinaciones de las WDLFs, las cuales se extienden a luminosidades y temperaturas más altas.

De los resultados del capítulo 4 , se puede concluir que los valores de $m_{a} \simeq 17 \mathrm{meV}$ inferidos de la astrosismología de las estrellas pulsantes G117-B15A y R548, ver Córsico et al. (2012a,b), se encuentran considerablemente desfavorecidas por el estudio de la WDLF. Es preciso notar que el elevado valor para la masa del axión derivado de estos últimos dos trabajos es una consecuencia directa de la identificación de los modos pulsacionales correspondientes a 215 s y 216 s de G117-B15A y R548 respectivamente, como modos atrapados en la envoltura. Consecuentemente, el resultado presentado en esta Tesis puede considerarse como un argumento firme para indicar que estos modos no se encuentran en realidad atrapados.

Al comparar las simulaciones teóricas con la WDLF de Miller Bertolami (2014), que incluye información para un rango de luminosidades extendido $\left(3<M_{B o l}<12.5\right)$, se ha encontrado que valores de $m_{a} \cos ^{2} \beta \gtrsim 10 \mathrm{meV}$ están en desacuerdo con dicha WDLF. Concretamente, el test de $\chi^{2}$ indica que valores de $m_{a} \cos ^{2} \beta \gtrsim 10 \mathrm{meV}$ se encuentran descartados a $3 \sigma$ de nivel de confianza. Desafortunadamente, no es posible asignar un nivel de confianza robusto a estos resultados debido a las discrepancias existentes entre las distintas WDLFs observadas y a la falta de una estimación de las incertezas en la tasa de formación estelar. Sin embargo, es preciso notar que las incertezas en las WDLFs 
podrían no ser relevantes. De hecho, cómputos numéricos como los desarrollados en Rowell (2013) sugieren que posibles fluctuaciones del orden del $50 \%$ en la SFR durante el último 1.5 Gyr se traducirían en una incerteza menor al $10 \%$ en la WDLF. Además, las incertezas actuales en los tiempos evolutivos de las EBs se encuentran por debajo del $10 \%$, ver Salaris et al. $(2013,2010)$, e implicarían incertezas similares en la WDLF. Es decir, no es de esperar que incertezas del orden del $10 \%$ en la WDLF teórica afecten significativamente a las cotas obtenidas en esta Tesis. Por otra parte, las discrepancias entre las distintas WDLF observadas son significativas. Se puede notar, por ejemplo, que mientras que la WDLF de Harris et al. (2006) no favorece el enfriamiento por axiones $\left(m_{a} \cos ^{2} \beta \lesssim 2.5 \mathrm{meV}\right)$, masas del axión menores a $m_{a} \cos ^{2} \beta \simeq 10 \mathrm{meV}$, por el contrario, no se encuentran excluidas por la WDLF del disco Galáctico. En particular, es importante destacar que ciertas características de algunas WDLFs, ver De Gennaro et al. (2008); Rowell \& Hambly (2011), pueden ser interpretadas como sugerencias de un enfriamiento por axiones DFSZ en el rango de masas $2.5 \mathrm{meV} \lesssim \mathrm{m}_{\mathrm{a}} \cos ^{2} \beta \lesssim 7.5 \mathrm{meV}$. Por otra parte, la cota aquí obtenida $\left(m_{a} \cos ^{2} \beta \gtrsim 10 \mathrm{meV}\right)$ resulta competitiva, siendo incluso aún más restrictiva, con aquella obtenida por Viaux et al. (2013) mediante el estudio de estrellas gigantes rojas en el cúmulo globular M5 (NGC 5904): $m_{a} \cos ^{2} \beta<$ $15 \mathrm{meV}$ al $95 \%$ de nivel de confianza (ver sección 2.5.1 y figura 2.3).

Por supuesto, para arribar a una cota definitiva para $m_{a} \cos ^{2} \beta$ por medio del estudio de la WDLF, es necesario continuar el trabajo aquí presentado. Desde el punto de vista de la determinación observacional de la WDLF, para estimar las posibles incertezas sistemáticas es necesario considerar WDLFs completamente independientes como aquellas presentadas por Krzesinski et al. (2009) y Rowell \& Hambly (2011). En particular, las nuevas WDLFs derivadas de SDSS-DR12, ver 201, incluirán un rango mucho más grande para las luminosidades de las EBs, lo cual mejorará considerablemente las cotas obtenidas. Desde el punto de vista teórico, es necesario un enfoque más complejo a fin de dar cuenta de las incertezas teóricas en la construcción de la WDLF, ver Geijo et al. (2006). Específicamente, deben considerarse las incertezas en la SFR, en la dinámica de la Galaxia y en el cociente de EBs ricas en $\mathrm{H}$ con respecto a las deficiente en $\mathrm{H}$. 



\section{A Código de Evolución Estelar LPCODE}

\section{A.1. Detalles Numéricos}

El código de evolución estelar utilizado en esta Tesis para el cálculo evolutivo de las simulaciones numéricas de EBs y Cefeidas es el LPCODE, ver Althaus et al. (2003), Althaus et al. (2005) y Althaus et al. (2012), el cual ha sido desarrollo íntegramente en la Facultad de Ciencias Astronómicas y Geofísicas. Este código ha sido utilizado recientemente para reproducir modelos de EBs muy precisos, ver García-Berro et al. (2010), Althaus et al. (2010a), Renedo et al. (2010) y Miller Bertolami et al. (2011) y sus referencias allí mencionadas. Asimismo, el código también ha sido empleado para estudiar la formación de estrellas subenanas a través del escenario de flashes calientes, ver Miller Bertolami et al. (2008), y el rol de la mezcla termohalina para la composición superficial de estrellas de baja masa, ver Wachlin et al. (2011). Es preciso mencionar que la descripción del código numérico LPCODE aquí presentada corresponde al código base, es decir no se incluyen los cambios que conciernen estrictamente a la presente Tesis en este apéndice.

Este código está basado, en líneas generales, en el método de Henyey tal como fue descripto en Kippenhahn et al. (1967). Para garantizar la estabilidad numérica, las ecuaciones del interior estelar son integradas en el código en un esquema de tipo Henyey 
totalmente implícito. Tales ecuaciones pueden ser escritas en formulación lagrangiana (con masa $m$ y tiempo $t$ como variables independientes) como sigue:

$$
\begin{aligned}
\frac{\partial r}{\partial m} & =\frac{1}{4 \pi r^{2} \rho} \\
\frac{\partial P}{\partial m} & =-\frac{G m}{4 \pi r^{4}} \\
\frac{\partial l}{\partial m} & =\epsilon_{n u c}-\epsilon_{\nu}-c_{P} \frac{\partial T}{\partial t}+\frac{\delta}{\rho} \frac{\partial P}{\partial t} \\
\frac{\partial T}{\partial m} & =-\frac{G m T}{4 \pi r^{4} P} \nabla \\
\frac{\partial X_{i}}{\partial t} & =F\left(X_{j}, T, P, r, l, \text { en todos los puntos de la estrella }\right)
\end{aligned}
$$

donde $r(m, t)$ es la distancia radial al centro de la estrella, $l(m, t)$ la luminosidad, $T(m, t)$ la temperatura, $P(m, t)$ la presión y $X_{i}$ representa las abundancias de los distintos elementos químicos. Asimismo, $\rho$ denota la densidad de masa, $\epsilon_{n u c}$ la energía nuclear liberada por unidad de masa y de tiempo, $\epsilon_{\nu}$ las pérdidas de energía por emisión de neutrinos por unidad de masa y de tiempo, $c_{P}$ es el calor específico a presión constante por unidad de masa), $\delta=\left(\frac{\partial \ln \rho}{\partial \ln T}\right)_{P}$ y $\nabla=\left(\frac{\partial \ln T}{\partial \ln P}\right)$ (gradiente adimensional de temperatura). Por último, los parámetros $G$ y c corresponden a las constantes de gravitación universal y a la velocidad de la luz en el vacío, respectivamente.

En el LPCODE las variables anteriores son cambiadas por otras que brindan mayor estabilidad numérica. El cambio de variables efectuado es el siguiente:

$$
\begin{array}{rlrl}
\xi & =\ln \left(1-m_{r} / M_{*}\right), & & \\
\theta & =\ln \left(T / T_{o}\right) & & T_{o} \equiv 10^{6} \mathrm{~K}, \\
p & =\ln \left(P / P_{o}\right) & & P_{o} \equiv 10^{15} \mathrm{din} \mathrm{cm}^{-2}, \\
x & =\ln \left(r / r_{o}\right) & r_{o} \equiv 10^{10} \mathrm{~cm}, \\
\lambda & =l / \Delta L_{o} & L_{o} \equiv 10^{33} \mathrm{erg} \mathrm{s}^{-1} .
\end{array}
$$

De esta manera, la variable independiente utilizada en el código ya no es la masa 
"m" sino $\xi$ y las variables dependientes son los logaritmos de las cantidades físicas involucradas, excepto por la luminosidad, en la cual el tamaño de $\lambda$ es regulado por la cantidad $\Delta$, la cual se reajusta automáticamente para que $\lambda$ permanezca por debajo del valor 10. Este tratamiento especial de la luminosidad hace que la misma pueda tomar valores negativos, lo cual es necesario ya que el gradiente de temperatura puede ser negativo y, por tanto, puede darse un flujo neto de energía hacia el interior estelar. Esto suele ocurrir en los interiores estelares como consecuencia del enfriamiento por neutrinos así como también cuando suceden combustiones violentas en algunas capas de las estrellas.

\section{A.2. Tratamiento De la ECUACión De CAMbio Químico}

Los cambios químicos de una región de la estrella son consecuencia de dos causas principalmente: las reacciones nucleares y los fenómenos de mezcla. En lo que sigue se mostrará como se analizan numéricamente estos procesos. A efectos de representar los cambios químicos utilizamos la variable $Y_{i}$ que se la puede escribir de la siguiente manera:

$$
Y_{i}=\frac{X_{i}}{A_{i}}\left(\propto \frac{\mathrm{n}^{\circ} \text { de particulas }}{\text { unidad de masa }}\right)
$$

donde $X_{i}$ es la fracción en masa del elemento bajo análisis y $A_{i}$ indica el peso atómico de esa misma especie. Si se incluye la variable $\lambda_{i j}$ como $\lambda_{i j}=\rho / m_{p}<\sigma v>_{i j}$ donde $m_{p}$ es la masa del protón y $\langle\sigma v>$ representa el valor medio respecto de la distribución de Maxwell del producto de la sección eficaz de la reacción nuclear y la velocidad relativa entonces los cambios químicos debidos a las reacciones nucleares resultan:

$$
\left(\frac{\partial Y_{i}}{\partial t}\right)_{\text {nuclear }}=-\sum_{j} \lambda_{i j} Y_{i} Y_{j}+\sum_{k, l} \lambda_{k l} Y_{k} Y_{l} . \quad i=1, \ldots, I
$$

La ec. (A.12) se trata en el código de evolución estelar utilizado en esta Tesis siguiendo un esquema implícito en diferencias finitas (método de Arnett y Truran 1969), donde se formula: 


$$
\frac{Y_{i}^{(n+1)}-Y_{i}^{(n)}}{\Delta t}=-\sum_{j} \lambda_{i j} Y_{i}^{(n+1)} Y_{j}^{(n+1)}+\sum_{k, l} \lambda_{k l} Y_{k}^{(n+1)} Y_{l}^{(n+1)},
$$

si se considera $Y_{i}^{(n+1)}=Y_{i}^{(n)}+\Delta Y$ y además si se aproxima a primer orden los siguientes términos

$$
Y_{i}^{(n+1)} Y_{j}^{(n+1)}=Y_{i}^{(n+1)} Y_{j}^{(n)}+Y_{i}^{(n)} Y_{j}^{(n+1)}-Y_{i}^{(n)} Y_{j}^{(n)}+\mathcal{O}\left(\Delta Y^{2}\right),
$$

se puede escribir, para los cambios químicos por reacciones nucleares, el siguiente sistema de ecuaciones algebraicas:

$$
\begin{aligned}
\frac{Y_{i}^{(n+1)}-Y_{i}^{(n)}}{\Delta t}= & -\left(\sum_{j} \lambda_{i j} Y_{i}^{(n+1)} Y_{j}^{(n)}+Y_{i}^{(n)} Y_{j}^{(n+1)}-Y_{i}^{(n)} Y_{j}^{(n)}\right)+ \\
+ & \left(\sum_{k, l} \lambda_{k l} Y_{k}^{(n+1)} Y_{l}^{(n)}+Y_{k}^{(n)} Y_{l}^{(n+1)}-Y_{k}^{(n)} Y_{l}^{(n)}\right)+\mathcal{O}\left(\Delta Y^{2}\right) \\
& \operatorname{para} i=1, \ldots, I
\end{aligned}
$$

el cual se puede expresar mediante notación vectorial como:

$$
\left(\frac{\vec{Y}^{n+1}-\vec{Y}^{n}}{\Delta t}\right)_{\mathrm{nuc}}=-\Gamma \vec{Y}^{n+1}+\vec{\Lambda}
$$

Aquí $\Gamma$ es una matriz de $I \times I$, donde $I$ representa el número total de especies consideradas. Además, $\Lambda$ es un vector columna de dimensión $I$ cuyos elementos tienen en cuenta a las abundancias $\left(Y_{i}^{(n)}\right)$ y a las tasas de las reacciones nucleares en el paso previo. Es importante destacar que los supraíndices que figuran en las ecuaciones anteriores indican el paso temporal de integración.

En lo que sigue se revisará el tratamiento de los cambios químicos debido a los procesos de mezcla entre las capas de la estrella. Kippenhahn \& Weigert (1990) realizan la aproximación clásica en evolución estelar, es decir, la aproximación de mezcla instantánea. Estos autores argumentan que la mezcla resulta muy eficiente en toda la zona 
convectiva y, por lo tanto, como las escalas de tiempo relativas a la convección son mucho más pequeñas que las asociadas a la quema nuclear, entonces la región convectiva resulta una región químicamente homogénea. En esta aproximación, la composición química se obtiene realizando un promedio capa a capa de las regiones involucradas. Sin embargo, cuando las escalas de tiempo de la mezcla convectiva son comparables a las escalas de tiempo de la quema nuclear, un tratamiento difusivo es generalmente empleado para determinar la evolución química (Salaris \& Cassisi (2017), Cloutman \& Eoll (1976)). Por lo tanto, en tales casos no se utiliza la aproximación de mezcla instantánea sino que se resuelve el problema dependiente del tiempo para los cambios químicos. Este es el enfoque seguido para el cálculo de las secuencias evolutivas utilizadas en esta Tesis; en particular sea ha tenido en cuenta la siguiente ecuación (Herwig (2000), Renedo et al. (2010), Freytag et al. (1996)):

$$
\left(\frac{d \vec{Y}}{d t}\right)=\left(\frac{\partial \vec{Y}}{\partial t}\right)_{\mathrm{nuc}}+\frac{\partial}{\partial M_{r}}\left[\left(4 \pi r^{2} \rho\right)^{2} D \frac{\partial \vec{Y}}{\partial M_{r}}\right]
$$

donde el primer término de la derecha considera los cambios debidos a las reacciones nucleares y el segundo a los cambios por procesos de mezcla. Asimismo, $\vec{Y}$ es un vector columna que contiene a las fracciones numéricas de las especies nucleares $\left(Y_{i}\right)$ y $D$ es el coeficiente de difusión que tiene en cuenta los procesos físicos involucrados en la mezcla de material tales como la convección, semiconvección e inestabilidades de Rayleigh Taylor (termohalina). El tratamiento numérico de la ec. (A.17) en el LPCODE se lleva a cabo mediante un esquema implícito de similares características que el adoptado para las reacciones nucleares, sin embargo, ahora además se realiza un desarrollo en diferencias finitas de la ecuación de difusión, la cual puede ser aproximada de la siguiente manera:

$$
\left\{\frac{\partial}{\partial M_{r}}\left[\left(4 \pi r^{2} \rho\right)^{2} D \frac{\partial \vec{Y}}{\partial M_{r}}\right]\right\}_{j}^{n+1} \approx \mathcal{A} \vec{Y}_{j-1}^{n+1}+\mathcal{B} \vec{Y}_{j}^{n+1}+\mathcal{C} \vec{Y}_{j+1}^{n+1}
$$

donde los subíndices indican la capa de la estrella. Las matrices $\mathcal{A}, \mathcal{B}, \mathcal{C}$ son múltiplos de la identidad de dimensión $I \times I$ cuyos elementos diagonales son: 


$$
\begin{gathered}
a_{i i}=\frac{(4 \pi)^{2}\left(\rho^{2} r^{4} D\right)_{j-1 / 2}}{\left(m_{j-1 / 2}-m_{j+1 / 2}\right)\left(m_{j-1}-m_{j}\right)}, \\
c_{i i}=\frac{(4 \pi)^{2}\left(\rho^{2} r^{4} D\right)_{j+1 / 2}}{\left(m_{j-1 / 2}-m_{j+1 / 2}\right)\left(m_{j}-m_{j+1}\right)}, \\
b_{i i}=-\left(a_{i i}+c_{i i}\right) .
\end{gathered}
$$

Es interesante notar que mediante este procedimiento se realiza un tratamiento simultáneo en los cambios químicos tanto por reacciones nucleares como por procesos de difusión. Cabe destacar que las expresiones anteriores son solamente válidas en las capas estelares pero no en los bordes. Para completar el conjunto de ecuaciones, se utiliza la condición de flujo de masa nulo y se obtiene el conjunto completo de ecuaciones para resolver el sistema. De esta manera, considerando las ecuaciones para los cambios químicos, tanto las debidas a las reacciones nucleares como a los fenómenos de mezcla se tiene:

$$
\left(\begin{array}{ccccccccc}
\mathcal{F}_{1} & \mathcal{C}_{2} & & & & & & & \\
\mathcal{A}_{1} & \mathcal{F}_{2} & \mathcal{C}_{3} & & & & & & \\
& \mathcal{A}_{2} & \mathcal{F}_{3} & \mathcal{C}_{4} & & & & & \\
& & \ddots & \ddots & \ddots & & & & \\
& & & \ddots & \ddots & \ddots & & & \\
& & & & \ddots & \ddots & \ddots & & \\
& & & & & \mathcal{A}_{J-3} & \mathcal{F}_{J-2} & \mathcal{C}_{J-1} & \\
& & & & & \mathcal{A}_{J-2} & \mathcal{F}_{J-1} & \mathcal{C}_{J} \\
& & & & & & \mathcal{A}_{J-1} & \mathcal{F}_{J}
\end{array}\right)\left(\begin{array}{c}
\vec{Y}_{1}^{n+1} \\
\vec{Y}_{2}^{n+1} \\
\vec{Y}_{3}^{n+1} \\
\vdots \\
\vdots \\
\vdots \\
\vec{Y}_{J-2}^{n+1} \\
\vec{Y}_{J-1}^{n+1} \\
\vec{Y}_{J}^{n+1}
\end{array}\right)=\left(\begin{array}{c}
\overrightarrow{\mathcal{M}}_{1} \\
\overrightarrow{\mathcal{M}}_{2} \\
\overrightarrow{\mathcal{M}}_{3} \\
\vdots \\
\vdots \\
\vdots \\
\overrightarrow{\mathcal{M}}_{J-2} \\
\overrightarrow{\mathcal{M}}_{J-1} \\
\overrightarrow{\mathcal{M}}_{J}
\end{array}\right)
$$

Donde $\mathcal{F}_{j}$ y $\overrightarrow{\mathcal{M}}_{j}$ están definidas como $\mathcal{F}_{j}=\mathcal{T}_{j}^{-1}+\Gamma_{j}-\mathcal{B}_{j}$ y $\overrightarrow{\mathcal{M}}_{j}=\vec{\Lambda}_{j}+\vec{Y}_{j}^{n} / \Delta t$. Por otra parte, las cantidades $\vec{\Lambda}_{j}$ y $\Gamma_{j}$ aparecen en la ec. (A.16) para los cambios químicos por reacciones nucleares y la matriz $\mathcal{T}_{j}^{-1}$ es un múltiplo de la matriz identidad de dimensión $N \times N$.

Para finalizar, es preciso decir que los cambios químicos y las ecuaciones de estructura estelar no se resuelven en forma simultánea en el LPCODE, ya que la química se obtiene en primer lugar. Sin embargo, una resolución simultánea de las ecuaciones de estructura 
y del cambio químico no es necesaria si el paso temporal se mantiene lo suficientemente chico como para que los cambios de estructura no sean muy grandes entre paso y paso, de manera que, en cada momento, los cambios químicos se computen con la estructura química correcta.

Para más detalles del código de evolución estelar LPCODE revisar Althaus et al. (2003), Althaus et al. (2005) y Althaus et al. (2012) y el apéndice A de Miller Bertolami (2009). 



\section{Referencias}

Abramowski, A., Acero, F., Aharonian, F., Ait Benkhali, F., Akhperjanian, A. G., Angüner, E., Anton, G., Balenderan, S., Balzer, A., Barnacka, A., \& et al.: 2013, Physical Review D 88(10), 102003

Adams: 1925, The Relativity Displacement of the Spectral Lines in the Companion of Sirius

Alexander, D. R. \& Ferguson, J. W.: 1994, Astrophysical Journal 437, 879

Althaus, L. G., Córsico, A. H., Bischoff-Kim, A., Romero, A. D., Renedo, I., GarcíaBerro, E., \& Miller Bertolami, M. M.: 2010a, Astrophysical Journal 717, 897

Althaus, L. G., Córsico, A. H., Isern, J., \& García-Berro, E.: 2010b, The Astronomy and Astrophysics Review 18, 471

Althaus, L. G., García-Berro, E., Isern, J., Córsico, A. H., \& Miller Bertolami, M. M.: 2012, Astronomy \& Astrophysics 537, A33

Althaus, L. G., Serenelli, A. M., Córsico, A. H., \& Montgomery, M. H.: 2003, Astronomy $\&$ Astrophysics 404, 593

Althaus, L. G., Serenelli, A. M., Panei, J. A., Córsico, A. H., García-Berro, E., \& Scóccola, C. G.: 2005, Astronomy \& Astrophysics 435, 631

Anderson, W.: 1929, Zeitschrift fur Physik 54, 433

Andriamonje, S., Aune, S., Autiero, D., Barth, K., Belov, A., Beltrán, B., Bräuninger, H., Carmona, J. M., Cebrián, S., Collar, J. I., Dafni, T., Davenport, M., Di Lella, L., Eleftheriadis, C., Englhauser, J., Fanourakis, G., Ferrer Ribas, E., Fischer, H., Franz, J., Friedrich, P., Geralis, T., Giomataris, I., Gninenko, S., Gómez, H., Hasinoff, M., 
Heinsius, F. H., Hoffmann, D. H. H., Irastorza, I. G., Jacoby, J., Jakovcic, K., Kang, D., Königsmann, K., Kotthaus, R., Krcmar, M., Kousouris, K., Kuster, M., Lakic, B., Lasseur, C., Liolios, A., Ljubicic, A., Lutz, G., Luzón, G., Miller, D., Morales, A., Morales, J., Ortiz, A., Papaevangelou, T., Placci, A., Raffelt, G., Riege, H., Rodríguez, A., Ruz, J., Savvidis, I., Semertzidis, Y., Serpico, P., Stewart, L., Vieira, J., Villar, J., Vogel, J., Walckiers, L., Zioutas, K., \& CAST Collaboration: 2007, Journal of Cosmology and Astroparticle Physics 4, 010

Angulo, C., Arnould, M., Rayet, M., Descouvemont, P., Baye, D., Leclercq-Willain, C., Coc, A., Barhoumi, S., Aguer, P., Rolfs, C., Kunz, R., Hammer, J. W., Mayer, A., Paradellis, T., Kossionides, S., Chronidou, C., Spyrou, K., degl'Innocenti, S., Fiorentini, G., Ricci, B., Zavatarelli, S., Providencia, C., Wolters, H., Soares, J., Grama, C., Rahighi, J., Shotter, A., \& Lamehi Rachti, M.: 1999, Nuclear Physics A 656, 3

Aprile, E., Agostini, F., Alfonsi, M., Arisaka, K., Arneodo, F., Auger, M., Balan, C., Barrow, P., Baudis, L., Bauermeister, B., Behrens, A., Beltrame, P., Bokeloh, K., Brown, A., Brown, E., Bruenner, S., Bruno, G., Budnik, R., Cardoso, J. M. R., Colijn, A. P., Contreras, H., Cussonneau, J. P., Decowski, M. P., Duchovni, E., Fattori, S., Ferella, A. D., Fulgione, W., Gao, F., Garbini, M., Geis, C., Goetzke, L. W., Grignon, C., Gross, E., Hampel, W., Itay, R., Kaether, F., Kessler, G., Kish, A., Landsman, H., Lang, R. F., Le Calloch, M., Lellouch, D., Levy, C., Lindemann, S., Lindner, M., Lopes, J. A. M., Lung, K., Lyashenko, A., Macmullin, S., Marrodán Undagoitia, T., Masbou, J., Massoli, F. V., Mayani Paras, D., Melgarejo Fernandez, A. J., Meng, Y., Messina, M., Miguez, B., Molinario, A., Murra, M., Naganoma, J., Ni, K., Oberlack, U., Orrigo, S. E. A., Pantic, E., Persiani, R., Piastra, F., Pienaar, J., Plante, G., Priel, N., Reichard, S., Reuter, C., Rizzo, A., Rosendahl, S., Dos Santos, J. M. F., Sartorelli, G., Schindler, S., Schreiner, J., Schumann, M., Scotto Lavina, L., Selvi, M., Shagin, P., Simgen, H., Teymourian, A., Thers, D., Tiseni, A., Trinchero, G., Vitells, O., Wang, H., Weber, M., Weinheimer, C., \& Xenon100 Collaboration: 2014, Physical Review D 90(6), 062009

Arvanitaki, A., Dimopoulos, S., Dubovsky, S., Kaloper, N., \& March-Russell, J.: 2010, Physical Review D 81(12), 123530 
Arvanitaki, A. \& Dubovsky, S.: 2011, Physical Review D 83(4), 044026

Asztalos, S. J., Bradley, R. F., Duffy, L., Hagmann, C., Kinion, D., Moltz, D. M., Rosenberg, L. J., Sikivie, P., Stoeffl, W., Sullivan, N. S., Tanner, D. B., van Bibber, K., \& Yu, D. B.: 2004, Physical Review D 69(1), 011101

Ayala, A., Domínguez, I., Giannotti, M., Mirizzi, A., \& Straniero, O.: 2014, Physical Review Letters 113(19), 191302

Baade, W.: 1926, Astronomische Nachrichten 228, 359

Baker, N. \& Kippenhahn, R.: 1962, Zeitschrift fur Astrophysik 54, 114

Ballou, R., Deferne, G., Finger, M., Finger, M., Flekova, L., Hosek, J., Kunc, S., Macuchova, K., Meissner, K. A., Pugnat, P., Schott, M., Siemko, A., Slunecka, M., Sulc, M., Weinsheimer, C., Zicha, J., \& Osqar Collaboration: 2015, Physical Review D 92(9), 092002

Bardeen, W. A. \& Tye, S.-H. H.: 1978, Physics Letters B 74, 229

Betz, M., Caspers, F., Gasior, M., Thumm, M., \& Rieger, S. W.: 2013, Physical Review D 88(7), 075014

Blout, B. D., Daw, E. J., Decowski, M. P., Ho, P. T. P., Rosenberg, L. J., \& Yu, D. B.: 2001, Astrophysical Journal 546, 825

Bono, G., Castellani, V., \& Marconi, M.: 2000, Astrophysical Journal 529, 293

Budker, D., Graham, P. W., Ledbetter, M., Rajendran, S., \& Sushkov, A. O.: 2014, Physical Review X 4(2), 021030

Burgers, J. M.: 1969, Flow Equations for Composite Gases

Caputo, F., Bono, G., Fiorentino, G., Marconi, M., \& Musella, I.: 2005, Astrophys. J. 629, 1021

Carosi, G., Friedland, A., Giannotti, M., Pivovaroff, M. J., Ruz, J., \& Vogel, J. K.: 2013, ArXiv e-prints 
Cassisi, S., Potekhin, A. Y., Pietrinferni, A., Catelan, M., \& Salaris, M.: 2007, Astrophysical Journal 661, 1094

Caughlan, G. R. \& Fowler, W. A.: 1988, Atomic Data and Nuclear Data Tables 40, 283

Chandrasekhar, S.: 1931, Astrophysical Journal 74, 81

Chandrasekhar, S.: 1939, An introduction to the study of stellar structure

Cheng, S. L., Geng, C. Q., \& Ni, W.-T.: 1995, Physical Review D 52, 3132

Cloutman, L. D. \& Eoll, J. G.: 1976, Astrophysical Journal 206, 548

Córsico, A. H., Althaus, L. G., Miller Bertolami, M. M., Romero, A. D., García-Berro, E., Isern, J., \& Kepler, S. O.: 2012a, Monthly Notices of the Royal Astronomical Society 424, 2792

Córsico, A. H., Althaus, L. G., Romero, A. D., Mukadam, A. S., García-Berro, E., Isern, J., Kepler, S. O., \& Corti, M. A.: 2012b, Journal of Cosmology and Astroparticle Physics 12, 010

Córsico, A. H., Benvenuto, O. G., Althaus, L. G., Isern, J., \& García-Berro, E.: 2001, New Astronomy 6, 197

De Gennaro, S., von Hippel, T., Winget, D. E., Kepler, S. O., Nitta, A., Koester, D., \& Althaus, L.: 2008, The Astronomical Journal 135, 1

Dicus, D. A., Kolb, E. W., Teplitz, V. L., \& Wagoner, R. V.: 1978, Physical Review D 18, 1829

Dine, M., Fischler, W., \& Srednicki, M.: 1981, Physics Letters B 104, 199

Dirac, P. A. M.: 1926, Proceedings of the Royal Society of London Series A 112, 661

Dufour, P., Liebert, J., Fontaine, G., \& Behara, N.: 2007, Nature 450, 522 
Eisenstein, D. J., Liebert, J., Harris, H. C., Kleinman, S. J., Nitta, A., Silvestri, N., Anderson, S. A., Barentine, J. C., Brewington, H. J., Brinkmann, J., Harvanek, M., Krzesiński, J., Neilsen, Jr., E. H., Long, D., Schneider, D. P., \& Snedden, S. A.: 2006, The Astrophysical Journal Supplement Series 167, 40

Ferguson, J. W., Alexander, D. R., Allard, F., Barman, T., Bodnarik, J. G., Hauschildt, P. H., Heffner-Wong, A., \& Tamanai, A.: 2005, Astrophysical Journal 623, 585

Fermi, E.: 1926, Zeitschrift fur Physik 36, 902

Fernie, J. D., Evans, N. R., Beattie, B., \& Seager, S.: 1995, Information Bulletin on Variable Stars 4148

Fowler, R. H.: 1926, Monthly Notices of the Royal Astronomical Society 87, 114

Freedman, W. L., Madore, B. F., Gibson, B. K., Ferrarese, L., Kelson, D. D., Sakai, S., Mould, J. R., Kennicutt, Jr., R. C., Ford, H. C., Graham, J. A., Huchra, J. P., Hughes, S. M. G., Illingworth, G. D., Macri, L. M., \& Stetson, P. B.: 2001, Astrophysical Journal 553,47

Freytag, B., Ludwig, H.-G., \& Steffen, M.: 1996, Astronomy \& Astrophysics 313, 497

Friedland, A., Giannotti, M., \& Wise, M.: 2013, Physical Review Letters 110(6), 061101

Fynbo, H. O. U., Diget, C. A., Bergmann, U. C., Borge, M. J. G., Cederkäll, J., Dendooven, P., Fraile, L. M., Franchoo, S., Fedosseev, V. N., Fulton, B. R., Huang, W., Huikari, J., Jeppesen, H. B., Jokinen, A. S., Jones, P., Jonson, B., Köster, U., Langanke, K., Meister, M., Nilsson, T., Nyman, G., Prezado, Y., Riisager, K., RintaAntila, S., Tengblad, O., Turrion, M., Wang, Y., Weissman, L., Wilhelmsen, K., Äystö, J., \& ISOLDE Collaboration: 2005, Nature 433, 136

Gänsicke, B. T., Koester, D., Girven, J., Marsh, T. R., \& Steeghs, D.: 2010, Science 327,188

García-Berro, E. \& Oswalt, T. D.: 2016, New Astronomy Reviews 72, 1 
García-Berro, E., Torres, S., Althaus, L. G., Renedo, I., Lorén-Aguilar, P., Córsico, A. H., Rohrmann, R. D., Salaris, M., \& Isern, J.: 2010, Nature 465, 194

Gautschy, A.: 1997, Vistas in Astronomy 41, 95

Geijo, E. M., Torres, S., Isern, J., \& García-Berro, E.: 2006, Monthly Notices of the Royal Astronomical Society 369, 1654

Giannotti, M., Irastorza, I., Redondo, J., \& Ringwald, A.: 2016, Journal of Cosmology and Astroparticle Physics 5, 057

Gill, R. \& Heyl, J. S.: 2011, Physical Review D 84(8), 085001

Goodricke, J.: 1786, Philosophical Transactions of the Royal Society of London Series I 76, 48

Green, R. F., Schmidt, M., \& Liebert, J.: 1986, Astrophysical Journal Supplement Series 61, 305

Guerrero, J., García-Berro, E., \& Isern, J.: 2004, Astronomy \& Astrophysics 413, 257

Hachisu, I., Kato, M., \& Nomoto, K.: 2012, Astrophysical Journal Letters 756, L4

Haft, M., Raffelt, G., \& Weiss, A.: 1994, Astrophysical Journal 425, 222

Halabi, G. M., Eid, M. F. E., \& Champagne, A.: 2012, The Astrophysical Journal $761(1), 10$

Halzen, F. \& Martin, A. D.: 1984, Quarks and leptons : an introductory course in modern particle physics

Harris, H. C., Munn, J. A., Kilic, M., Liebert, J., Williams, K. A., von Hippel, T., Levine, S. E., Monet, D. G., Eisenstein, D. J., Kleinman, S. J., Metcalfe, T. S., Nitta, A., Winget, D. E., Brinkmann, J., Fukugita, M., Knapp, G. R., Lupton, R. H., Smith, J. A., \& Schneider, D. P.: 2006, The Astronomical Journal 131, 571

Herwig, F.: 2000, Astronomy \& Astrophysics 360, 952 
Horns, D., Maccione, L., Mirizzi, A., \& Roncadelli, M.: 2012, Physical Review D 85(8), 085021

Hudson, H. S., Acton, L. W., DeLuca, E. E., Hannah, I. G., Reardon, K., \& Van Bibber, K.: 2012, in L. Bellot Rubio, F. Reale, \& M. Carlsson (eds.), 4th Hinode Science Meeting: Unsolved Problems and Recent Insights, Vol. 455 of Astronomical Society of the Pacific Conference Series, p. 25

Humason, M. L. \& Zwicky, F.: 1947, Astrophysical Journal 105, 85

Iben, Jr., I. \& Laughlin, G.: 1989, Astrophysical Journal 341, 312

Iglesias, C. A. \& Rogers, F. J.: 1996, Astrophysical Journal 464, 943

Isern, J., Artigas, A., \& García-Berro, E.: 2013, in European Physical Journal Web of Conferences, Vol. 43 of European Physical Journal Web of Conferences, p. 05002

Isern, J., Catalan, S., Garcia-Berro, E., Salaris, M., \& Torres, S.: 2010, ArXiv e-prints

Isern, J. \& García-Berro, E.: 2008, Memorie della Società Astronomica Italiana 79, 545

Isern, J., García-Berro, E., Hernanz, M., Mochkovitch, R., \& Torres, S.: 1998, Astrophysical Journal 503, 239

Isern, J., García-Berro, E., Torres, S., \& Catalán, S.: 2008, Astrophysical Journal Letters 682, L109

Isern, J., Mochkovitch, R., García-Berro, E., \& Hernanz, M.: 1997, Astrophysical Journal 485, 308

Itoh, N., Hayashi, H., Nishikawa, A., \& Kohyama, Y.: 1996, Astrophysical Journal, Supplement 102, 411

Kepler, S. O., Koester, D., Romero, A. D., Ourique, G., \& Pelisoli, I.: 2017, in P.-E. Tremblay, B. Gaensicke, \& T. Marsh (eds.), 20th European White Dwarf Workshop, Vol. 509 of Astronomical Society of the Pacific Conference Series, p. 421 
Kepler, S. O., Pelisoli, I., Koester, D., Ourique, G., Kleinman, S. J., Romero, A. D., Nitta, A., Eisenstein, D. J., Costa, J. E. S., Külebi, B., Jordan, S., Dufour, P., Giommi, P., \& Rebassa-Mansergas, A.: 2015, Monthly Notices of the Royal Astronomical Society 446, 4078

Kepler, S. O., Pelisoli, I., Koester, D., Ourique, G., Romero, A. D., Reindl, N., Kleinman, S. J., Eisenstein, D. J., Valois, A. D. M., \& Amaral, L. A.: 2016, Monthly Notices of the Royal Astronomical Society 455, 3413

Kim, J. E.: 1979, Physical Review Letters 43, 103

Kippenhahn, R. \& Weigert, A.: 1990, Stellar Structure and Evolution

Kippenhahn, R., Weigert, A., \& Hofmeister, E.: 1967, Methods in Computational Physics 7, 129

Kleinman, S. J., Kepler, S. O., Koester, D., Pelisoli, I., Peçanha, V., Nitta, A., Costa, J. E. S., Krzesinski, J., Dufour, P., Lachapelle, F.-R., Bergeron, P., Yip, C.-W., Harris, H. C., Eisenstein, D. J., Althaus, L., \& Córsico, A.: 2013, The Astrophysical Journal Supplement 204, 5

Krzesinski, J., Kleinman, S. J., Nitta, A., Hügelmeyer, S., Dreizler, S., Liebert, J., \& Harris, H.: 2009, Astronomy \& Astrophysics 508, 339

Kunz, R., Fey, M., Jaeger, M., Mayer, A., Hammer, J. W., Staudt, G., Harissopulos, S., \& Paradellis, T.: 2002, The Astrophysical Journal 567, 643

Leggett, S. K., Ruiz, M. T., \& Bergeron, P.: 1998, Astrophysical Journal 497, 294

Liebert, J., Bergeron, P., \& Holberg, J. B.: 2005, The Astrophysical Journal Supplement Series 156, 47

Magni, G. \& Mazzitelli, I.: 1979, Astronomy and Astrophysics 72, 134

Melendez, B. E., Miller Bertolami, M. M., \& Althaus, L. G.: 2014, in Revista Mexicana de Astronomia y Astrofisica Conference Series, Vol. 44 of Revista Mexicana de Astronomia y Astrofisica Conference Series, pp 48-48 
Miller Bertolami, M. M.: 2009, Ph.D. thesis, Facultad de Ciencias Astronómicas y Geofísicas

Miller Bertolami, M. M.: 2014, Astronomy \& Astrophysics 562, A123

Miller Bertolami, M. M., Althaus, L. G., Unglaub, K., \& Weiss, A.: 2008, Astronomy E3 Astrophysics 491, 253

Miller Bertolami, M. M., Melendez, B. E., Althaus, L. G., \& Isern, J.: 2014, Journal of Cosmology and Astroparticle Physics 10, 069

Miller Bertolami, M. M., Melendez, B. E., Althaus, L. G., \& Isern, J.: 2015, in P. Dufour, P. Bergeron, \& G. Fontaine (eds.), 19th European Workshop on White Dwarfs, Vol. 493 of Astronomical Society of the Pacific Conference Series, p. 133

Miller Bertolami, M. M., Rohrmann, R. D., Granada, A., \& Althaus, L. G.: 2011, Astrophysical Journal Letters 743, L33

Nakagawa, M., Adachi, T., Kohyama, Y., \& Itoh, N.: 1988, Astrophysical Journal 326, 241

Nakagawa, M., Kohyama, Y., \& Itoh, N.: 1987, Astrophysical Journal 322, 291

Neilson, H. R., Langer, N., Engle, S. G., Guinan, E., \& Izzard, R.: 2012, The Astrophysical Journal Letters $\mathbf{7 6 0}$, L18

Olive, K. A. et al.: 2014, Chin. Phys. C38, 090001

Patrignani, C. et al.: 2016, Chin. Phys. C40(10), 100001

Paxton, B., Cantiello, M., Arras, P., Bildsten, L., Brown, E. F., Dotter, A., Mankovich, C., Montgomery, M. H., Stello, D., Timmes, F. X., \& Townsend, R.: 2013, The Astrophysical Journal Supplement 208, 4

Payez, A., Cudell, J. R., \& Hutsemékers, D.: 2012, Journal of Cosmology and Astroparticle Physics 7, 041

Payez, A., Evoli, C., Fischer, T., Giannotti, M., Mirizzi, A., \& Ringwald, A.: 2015, Journal of Cosmology and Astroparticle Physics 2, 006 
Peccei, R. D. \& Quinn, H. R.: 1977a, Physical Review D 16, 1791

Peccei, R. D. \& Quinn, H. R.: 1977b, Physical Review Letters 38, 1440

Pietrinferni, A., Cassisi, S., Salaris, M., \& Castelli, F.: 2004, Astrophysical Journal 612, 168

Pound, R. V. \& Rebka, G. A.: 1959, Physical Review Letters 3, 439

Primakoff, H.: 1951, Physical Review 81, 899

Quinn, H.: 2001, ArXiv High Energy Physics - Phenomenology e-prints, hep$\mathrm{ph} / 0110050$

Raffelt, G. \& Weiss, A.: 1995, Physical Review D 51, 1495

Raffelt, G. G.: 1986, Physics Letters B 166, 402

Raffelt, G. G.: 1990, Physics Reports 198, 1

Raffelt, G. G.: 1996, Stars as laboratories for fundamental physics : the astrophysics of neutrinos, axions, and other weakly interacting particles, University of Chicago Press.

Raffelt, G. G.: 1999, Annual Review of Nuclear and Particle Science 49, 163

Raffelt, G. G.: 2008, in M. Kuster, G. Raffelt, \& B. Beltrán (eds.), Axions, Vol. 741 of Lecture Notes in Physics, Berlin Springer Verlag, p. 51

Raffelt, G. G. \& Dearborn, D. S. P.: 1988, Physical Review D 37, 549

Renedo, I., Althaus, L. G., Miller Bertolami, M. M., Romero, A. D., Córsico, A. H., Rohrmann, R. D., \& García-Berro, E.: 2010, Astrophysical Journal 717, 183

Renzini, A., Greggio, L., Ritossa, C., \& Ferrario, L.: 1992, Astrophysical Journal 400, 280

Rohrmann, R. D., Althaus, L. G., García-Berro, E., Córsico, A. H., \& Miller Bertolami, M. M.: 2012, Astronomy \& Astrophysics 546, A119

Rowell, N.: 2013, Monthly Notices of the Royal Astronomical Society 434, 1549 
Rowell, N. \& Hambly, N. C.: 2011, Monthly Notices of the Royal Astronomical Society 417,93

Salaris, M., Althaus, L. G., \& García-Berro, E.: 2013, Astronomy \&3 Astrophysics 555, A96

Salaris, M. \& Cassisi, S.: 2005, Evolution of stars and stellar populations, J. Wiley

Salaris, M. \& Cassisi, S.: 2006, Evolution of Stars and Stellar Populations

Salaris, M. \& Cassisi, S.: 2017, ArXiv e-prints, 1707.07454

Salaris, M., Cassisi, S., Pietrinferni, A., Kowalski, P. M., \& Isern, J.: 2010, Astrophysical Journal 716, 1241

Salaris, M., Serenelli, A., Weiss, A., \& Miller Bertolami, M.: 2009, Astrophysical Journal 692, 1013

Sato, K. \& Sato, H.: 1975, Progress of Theoretical Physics 54, 1564

Segretain, L., Chabrier, G., Hernanz, M., Garcia-Berro, E., Isern, J., \& Mochkovitch, R.: 1994, Astrophysical Journal 434, 641

Semertzidis, Y., Cameron, R., Cantatore, G., Melissinos, A. C., Rogers, J., Halama, H., Prodell, A., Nezrick, F., Rizzo, C., \& Zavattini, E.: 1990, Physical Review Letters 64, 2988

Shapley, H.: 1914, Astrophysical Journal 40, 448

Shifman, M. A., Vainshtein, A. I., \& Zakharov, V. I.: 1980, Nuclear Physics B 166, 493

Siess, L.: 2007, Astronomy \& Astrophysics 476, 893

Sikivie, P.: 1983, Physical Review Letters 51, 1415

Soszyński, I., Udalski, A., Szymański, M. K., Kubiak, M., Pietrzyński, G., Wyrzykowski, L., Szewczyk, O., Ulaczyk, K., \& Poleski, R.: 2008, Acta Astronomica 58, 293 
Stoner, E.: 1930, The London, Edinburgh, and Dublin Philosophical Magazine and Journal of Science: Series 7, Volume 9, Issue 60, p. 944-963 9, 944

Szabados, L.: 2003, in U. Munari (ed.), GAIA Spectroscopy: Science and Technology, Vol. 298 of Astronomical Society of the Pacific Conference Series, p. 237

Szabados, L.: 2010, Odessa Astronomical Publications 23, 106

Turner, M. S.: 1990, Physics Reports 197, 67

van Bibber, K., McIntyre, P. M., Morris, D. E., \& Raffelt, G. G.: 1989, Phys. Rev. D 39, 2089

Viaux, N., Catelan, M., Stetson, P. B., Raffelt, G. G., Redondo, J., Valcarce, A. A. R., \& Weiss, A.: 2013, Physical Review Letters 111(23), 231301

Vinyoles, N., Serenelli, A., Villante, F. L., Basu, S., Redondo, J., \& Isern, J.: 2015, Journal of Cosmology and Astroparticle Physics 10, 015

Wachlin, F. C., Miller Bertolami, M. M., \& Althaus, L. G.: 2011, Astronomy \& Astrophysics 533, A139

Weidemann, V.: 1968, Annual Review of Astronomy and Astrophysics 6, 351

Wesselink, A. J.: 1946, Bulletin of the Astronomical Institutes of the Netherlands 10, 91

Wise, M. B., Georgi, H., \& Glashow, S. L.: 1981, Physical Review Letters 47, 402

York, D. G., Adelman, J., Anderson, Jr., J. E., Anderson, S. F., Annis, J., Bahcall, N. A., Bakken, J. A., Barkhouser, R., Bastian, S., Berman, E., Boroski, W. N., Bracker, S., Briegel, C., Briggs, J. W., Brinkmann, J., Brunner, R., Burles, S., Carey, L., Carr, M. A., Castander, F. J., Chen, B., Colestock, P. L., Connolly, A. J., Crocker, J. H., Csabai, I., Czarapata, P. C., Davis, J. E., Doi, M., Dombeck, T., Eisenstein, D., Ellman, N., Elms, B. R., Evans, M. L., Fan, X., Federwitz, G. R., Fiscelli, L., Friedman, S., Frieman, J. A., Fukugita, M., Gillespie, B., Gunn, J. E., Gurbani, V. K., de Haas, E., Haldeman, M., Harris, F. H., Hayes, J., Heckman, T. M., Hennessy, G. S., Hindsley, 
R. B., Holm, S., Holmgren, D. J., Huang, C.-h., Hull, C., Husby, D., Ichikawa, S.-I., Ichikawa, T., Ivezić, Ž., Kent, S., Kim, R. S. J., Kinney, E., Klaene, M., Kleinman, A. N., Kleinman, S., Knapp, G. R., Korienek, J., Kron, R. G., Kunszt, P. Z., Lamb, D. Q., Lee, B., Leger, R. F., Limmongkol, S., Lindenmeyer, C., Long, D. C., Loomis, C., Loveday, J., Lucinio, R., Lupton, R. H., MacKinnon, B., Mannery, E. J., Mantsch, P. M., Margon, B., McGehee, P., McKay, T. A., Meiksin, A., Merelli, A., Monet, D. G., Munn, J. A., Narayanan, V. K., Nash, T., Neilsen, E., Neswold, R., Newberg, H. J., Nichol, R. C., Nicinski, T., Nonino, M., Okada, N., Okamura, S., Ostriker, J. P., Owen, R., Pauls, A. G., Peoples, J., Peterson, R. L., Petravick, D., Pier, J. R., Pope, A., Pordes, R., Prosapio, A., Rechenmacher, R., Quinn, T. R., Richards, G. T., Richmond, M. W., Rivetta, C. H., Rockosi, C. M., Ruthmansdorfer, K., Sandford, D., Schlegel, D. J., Schneider, D. P., Sekiguchi, M., Sergey, G., Shimasaku, K., Siegmund, W. A., Smee, S., Smith, J. A., Snedden, S., Stone, R., Stoughton, C., Strauss, M. A., Stubbs, C., SubbaRao, M., Szalay, A. S., Szapudi, I., Szokoly, G. P., Thakar, A. R., Tremonti, C., Tucker, D. L., Uomoto, A., Vanden Berk, D., Vogeley, M. S., Waddell, P., Wang, S.-i., Watanabe, M., Weinberg, D. H., Yanny, B., Yasuda, N., \& SDSS Collaboration: 2000, The Astronomical Journal 120, 1579

Zhitnitskii, A.: 1980, Sov. J. Nucl. Phys. (Engl. Transl.); (United States) 31:2

Zhitnitsky, A. R.: 1980, Sov. J. Nucl. Phys. 31, 260, [Yad. Fiz.31,497(1980)] 



\section{Índice de figuras}

1.1. Función distribución de masa para 3636 EB DAs con $T_{\text {eff }}>13000 \mathrm{~K}$ representadas por líneas negras y 549 EB DBs con $T_{\text {eff }}>16000 \mathrm{~K}$ representadas por líneas rojas, ver 201. . . . . . . . . . . .

1.2. Diagrama H-R para la evolución completa de una estrella de $3.5 \mathrm{M}_{\odot}$ desde la ZAMS hasta el estadio de EB. Se indican también los distintos tipos de enfriamiento y las bandas de inestabilidad para las EB pulsantes DOV, DBV y DAV. Figura tomada de Althaus et al. (2010b). . . . . . . . . . .

1.3. Comparación de la WDLF del disco derivada del Sloan Digital Sky Survey (SDSS) en Harris et al. (2006) con aquellas obtenidas en Leggett et al. (1998) y Liebert et al. (2005). Figura tomada de Althaus et al. (2010b) .

1.4. Ubicación de la banda de inestabilidad de las Cefeidas en el diagrama H-R. Las etiquetas indican la zona de inestabilidad de los modos pulsacionales. F indica el modo fundamental de pulsación, FO el primer fundamental, FOBE el límite azul para la región pulsacional, FRE el límite rojo de la misma, FBE el límite azul para el fundamental y FORE indica el límite rojo para el primer fundamental. Se muestran además las líneas de período constante. Figura extraída de Salaris \& Cassisi (2006). . . . . . . . . . .

1.5. Diagrama Color-Magnitud para Cefeidas Clásicas en la Nube Mayor de Magallanes. Las estrellas de fondo (puntos negros) pertenecen al subcampo LMC100.1. Los distintos colores representan Cefeidas que están pulsando en un modo particular. Los puntos azul oscuro representan los modos fundamentales de pulsación, los rojos el primer armónico y los verdes el segundo armónico. Los puntos azul claro representan modos de pulsación mixtos. Datos extraídos del relevamiento OGLE, ver Soszyński et al. (2008). 23 
1.6. Evolución en el diagrama HR para estrellas con masas entre $7 M_{\odot}-12 M_{\odot}$. La línea puntea representa la banda de inestabilidad de las Cefeidas, tomado de Friedland et al. (2013). . . . . . . . . . . . . . . . . . . . . . . . 24

2.1. Diagrama que representa la mezcla del axión con el pión neutro. Las lineas curvas representan a los gluones y las rectas a los quarks, ver Raffelt (1996). 29

2.2. Conversión Primakoff entre axiones con fotones en un campo electromagnético externo, ver Raffelt (1996). . . . . . . . . . . . . . . 33

2.3. Rangos de exclusión como se describen en el texto. Los intervalos en la fila de abajo son los rangos de búsqueda aproximados de los experimentos ADMX, CASPEr, CAST, e IAXO, donde las regiones verdes indican las búsquedas proyectadas. Los límites en las constantes de acoplamientos se trasladan a $m_{a}$ y $f_{a}$ utilizando $z=0.56$ y los valores para el modelo KSVZ, a menos que se mencione lo contrario. Los límites para $g_{a e}$ están determinados por el modelo DFSZ con un acoplamiento axión-electrón correspondiente a $\cos \beta \boldsymbol{\prime}=1 / 2$. Figura extraída de Patrignani et al. (2016). 42

2.4. Figura que muestra los rangos de exclusión para las ALPs, como se describen en el texto, ver Patrignani et al. (2016). . . . . . . . . . . . . . .

3.1. Consecuencias del enfriamiento anómalo por axiones en la evolución de una estrella de $9.5 \mathrm{M}_{\odot}$ al usar los mismos parámetros físicos que en el trabajo de Carosi et al. (2013). Se muestran los perfiles de temperatura efectiva correspondientes a $g_{10}=0.0,0.6,1.2,2$ y $3 \ldots \ldots \ldots$

3.2. Secuencias evolutivas para una estrella representativa de $9.5 M_{\odot}$ con los mismos parámetros de overshooting que los utilizados por Friedland et al. (2013) y Carosi et al. (2013) con $g_{10}=0,0.6,1.2,2$ y $3 . \ldots 56$

3.3. Duración de la quema central de He en Myr para estrellas en el rango de $6 M_{\odot} \leq M \leq 10 M_{\odot}$ en función de la constante de acoplamiento axiónfotón $g_{10}$ teniendo en cuenta los siguientes parámetros de overshooting: $f_{n u c}=0.0174$ y $f_{\text {env }}=0.0174$ durante la quema de $\mathrm{H}$ en el núcleo, $f_{\text {nuc }}=$ 0.0174 y $f_{\text {env }}=0.0174$ durante la quema de He. . . . . . . . . . . . 
3.4. Secuencias evolutivas correspondientes al SET1 de parámetros para una estrella de $7 M_{\odot}$ con $g_{10}=0,0.6,0.8,1,1.5,2$ y 2.5. La desaparición del blue loop ocurre para la secuencia correspondiente a la constante de acoplamiento $g_{10}=2.5 \ldots \ldots \ldots \ldots \ldots \ldots$

3.5. Evolución en el diagrama H-R para estrellas en el rango $6 M_{\odot}-10 M_{\odot}$ para el SET5 de parámetros con el agregado de un enfriamiento anómalo correspondiente a $g_{10}=2 \ldots \ldots \ldots \ldots \ldots \ldots$

4.1. Emisión de fotones (línea roja), axiones (curvas negras) y de neutrinos (curvas azules) para la secuencia de $0.609 M_{\odot}$ asumiendo distintas masas para el axión. El impacto de la emisión de axiones en la estructura térmica de la EB se evidencia en el decrecimiento de la emisión de neutrinos para altas luminosidades. Claramente, la emisión de axiones no puede ser tratada de manera perturbativa para $m_{a} \cos ^{2} \beta>5 \mathrm{meV} \ldots \ldots$. . . . .

4.2. Comparación de la emisión de axiones $\left(m_{a} \cos ^{2} \beta=5 \mathrm{meV}\right)$ y la emisión de neutrinos de la secuencia de $0.609 M_{\odot}$ realizada en este trabajo con la secuencia de $0.61 M_{\odot}$ de Isern et al. (2008). La línea sólida negra corresponde a la luminosidad de neutrinos (desde arriba hacia abajo: $m_{a} \cos ^{2} \beta=$ 0,5 meV) del modelo presentado en este capítulo. La línea sólida roja representa la emisión de neutrinos del modelo de Isern, la línea punteada negra la emisión de axiones para $5 \mathrm{meV}$ de este trabajo y la línea punteada roja la emisión de axiones para $5 \mathrm{meV}$ del modelo de Isern et al. (2008). Finalmente, la línea azul representa la luminosidad de fotones. Los efectos del apartamiento de la aproximación de núcleo isotérmico son apreciables para $M_{b o l}<7 \ldots \ldots \ldots \ldots \ldots \ldots \ldots \ldots \ldots \ldots \ldots$

4.3. WDLFs del disco Galáctico adoptadas a fines comparativos. La WDLF de Rowell \& Hambly (2011) ha sido multiplicada por un factor de 1.862 como se describe en Miller Bertolami (2014). . . . . . . . . . . . . . . 
4.4. WDLFs construidas para distintas masas del axión comparadas con la WDLF de Harris et al. (2006). Los axiones DFSZ con $m_{a}>10 \mathrm{meV}$ están claramente excluidos por la WDLF observada. Es posible notar que las WDLF teóricas construidas con $m_{a}>10 \mathrm{meV}$ están casi superpuestas en el rango de interés. Esto se debe al hecho de que la región de normalización es, en esos casos, también afectada por la emisión de axiones. . . . . . .

4.5. Valores de $\chi^{2}$ (mapa de color) derivados de la comparación de las WDLFs teóricas con aquellas obtenidas por (de arriba hacia abajo) Harris et al. (2006), De Gennaro et al. (2008), Rowell \& Hambly (2011) y Isern et al. (2008) para distintas elecciones del punto de normalización (eje y) y la masa del axión $($ eje $\mathrm{x}) \ldots \ldots . \ldots$. . . . . . . . . . . 73

4.5. Continuación . . . . . . . . . . . . . . . . . 74

4.6. Comparación de la WDLF construida por Miller Bertolami (2014) con la WDLF teórica para distintas masas del axión. La línea continua en el recuadro interior muestra el nivel de confianza con el cual los distintos valores de la masa del axión son descartados por el test $\chi^{2}$. Por referencia se indican mediante líneas discontinuas los niveles de significancia asociados

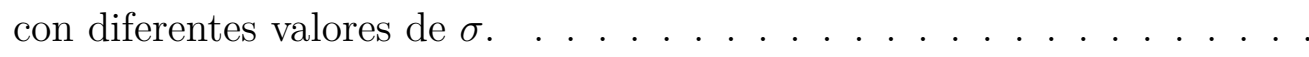




\section{Indíce de Tablas}

2.1. Valores y cotas para los momentos dipolares eléctricos y magnéticos extraídos de Olive et al. (2014), "The Review of Particle Physics (2015)". $\mu_{B}$ es el magnetón de Bohr y $\mu_{N}$ es el magnetón nuclear. . . . . . . . . .

3.1. Tiempos evolutivos en Myr durante la quema central de $\mathrm{H}$ y He obtenidos con los códigos de evolución estelar LPCODE y MESA para estrellas con $M=3-8 M_{\odot} \ldots \ldots \ldots \ldots \ldots \ldots$

3.2. Definición de los SETs de parámetros en términos del overshooting nuclear y en la envoltura. . . . . . . . . . . . . . . . . . . . .

3.3. Valor de la cota para $g_{10}$ obtenido para cada masa estelar con cada uno de los conjuntos de parámetros definidos en la tabla 3.2 . . . . . . . . . 